\title{
Treatment of basal cell carcinoma in the light of photodynamic therapy
}

Citation for published version (APA):

Thissen, M. R. T. M. (2000). Treatment of basal cell carcinoma in the light of photodynamic therapy.

[Doctoral Thesis, Maastricht University]. Universiteit Maastricht. https://doi.org/10.26481/dis.20000623mt

Document status and date:

Published: 01/01/2000

DOI:

10.26481/dis.20000623mt

Document Version:

Publisher's PDF, also known as Version of record

\section{Please check the document version of this publication:}

- A submitted manuscript is the version of the article upon submission and before peer-review. There can be important differences between the submitted version and the official published version of record.

People interested in the research are advised to contact the author for the final version of the publication, or visit the DOI to the publisher's website.

- The final author version and the galley proof are versions of the publication after peer review.

- The final published version features the final layout of the paper including the volume, issue and page numbers.

Link to publication

\footnotetext{
General rights rights.

- You may freely distribute the URL identifying the publication in the public portal. please follow below link for the End User Agreement:

www.umlib.nl/taverne-license

Take down policy

If you believe that this document breaches copyright please contact us at:

repository@maastrichtuniversity.nl

providing details and we will investigate your claim.
}

Copyright and moral rights for the publications made accessible in the public portal are retained by the authors and/or other copyright owners and it is a condition of accessing publications that users recognise and abide by the legal requirements associated with these

- Users may download and print one copy of any publication from the public portal for the purpose of private study or research.

- You may not further distribute the material or use it for any profit-making activity or commercial gain

If the publication is distributed under the terms of Article $25 \mathrm{fa}$ of the Dutch Copyright Act, indicated by the "Taverne" license above, 
Treatment of Basal Cell Carcinoma

in the Light of Photodynamic Therapy 
M.R.T.M. Thissen, Maastricht 2000

ISBN 90-5681-073-1

Vormgeving en druk: Unigraphic, Universiteit Maastricht. 


\title{
Treatment of Basal Cell Carcinoma in the Light of Photodynamic Therapy
}

\author{
PROEFSCHRIFT \\ ter verkrijging van de graad doctor aan \\ de Universiteit Maastricht, \\ op gezag van de Rector Magnificus, \\ Prof.dr. A.C. Nieuwenhuijzen Kruseman, \\ volgens het besluit van het College van Decanen, \\ in het openbaar te verdedigen \\ op vrijdag 23 juni 2000 om 14.00 uur
} door

Monique Rosalie Thérèse Mathieu Thissen 


\section{Promotor}

Prof.dr. H.A.M. Neumann

\section{Beoordelingscommissie}

Prof.dr. J.J. Manni (voorzitter)

Prof.dr. W.D. Boeckx

Prof.dr. M. Borgers

Prof.dr.ir. M.J.C. van Gemert (Academisch Medisch Centrum, Amsterdam)

Dr. W.M. Star (Daniel den Hoed Kliniek, Rotterdam)

Financial support by 3M Pharma Nederland BV, Stiefel Laboratories BV, Janssen-Cilag BV, Varodem, Galderma, Yamanouchi Pharma BV, Glaxo Wellcome BV, ABN-AMRO, Leo Pharmaceutical Products BV, medi Nederland BV, Novartis Pharma BV, Beiersdorf NV, Bipharma BV, Roche Nederland BV for the publication of this thesis is gratefully acknowledged. 
Anderen kennen is Wijsheid, Jezelf kennen is Verlichting

(Tao te tsing)

Aan mijn Ouders, Voor Jack 



\section{CONTENTS}

Chapter 1: The epidemiology of basal cell carcinoma.

Chapter 2: Treatment modalities for basal cell carcinoma; a light thrown upon surgical interventions, cryosurgery and photodynamic therapy.

Chapter 3: A systematic review of treatment madalities for primary basal cell carcinoma.

Chapter 4: Cosmetic results of cryosurgery versus conventional surgical excision for treatment of primary, uncomplicated basal cell carcinoma of the head and neck.

Chapter 5: Treatment of basal cell carcinoma by dermatologists in the Netherlands.

Chapter 6: Effective photodynamic therapy with 5-aminolevulinic acid for nodular basal cell carcinoma, using a preceding debulking technique.

Chapter 7: In vivo pharmacokinetics of PpLX accumulation following intracutaneous injection of 5-aminoleurlinic acid for photodynamic therapy; investigations of a doseresponse relationship.

Chapter 8: $\quad P p D X$ fluorescence kinetics and increased damage after intracutaneous injection of 5-aminolevulinic acia and repeated illumination.

Chapter 9: General discussion and Recommendations.

Samenvatting 
GENERAL INTRODUCTION 
Basal cell carcinoma $(\mathrm{BCC})$, as we know it today, already dates back to 2000 BC. Evidence of its existence was furnished by excavations in Egypt, where bony manifestations related to the nevoid basal cell carcinoma syndrome were found in mummified skeletons (Satinoff, 1969). Throughout all ages the tumour has been described with several terms, including for example noli-me-tangere (touch-me-not) (Marmelzat, 1964), ulcus rodens (Bennett, 1974) and Jacobs ulcer (Jacob, 1827). In the beginning of the 20th century, Krompecher was the first one who clearly distinguished this tumour from other epithelial tumours and gave it the name of "Basalzellen-Krebs" (Krompecher, 1903). From that moment on, the tumour became gradually known because of the rising incidence. $\mathrm{BCC}$ has been the most common skin cancer all over the world for many years. Incidence rates are still increasing (Marks, 1995), which is the result of an increase in sun exposure, in combination with the increase in the average age of the population. BCC rarely metastasizes, but the malignant character is determined especially by local invasion and tissue destruction, leading to serious disfigurement. Although mortality for $\mathrm{BCC}$ is low, the high prevalence and the high morbidity have made $\mathrm{BCC}$ to both a serious and costly health problem (Dunn,1965; Preston,1992). Furthermore, lesions may recur, even leading to more difficulties. Patients are also developing multiple tumours, giving major implications for treatment and followup.

For the treatment of $\mathrm{BCC}$, several surgical and non-surgical therapies are available. In the literature, recurrence rates after treatment of primary $\mathrm{BCC}$ for these different therapies vary between $0.7-10.1 \%$ for surgical excision, 3.3-7.7\% for curettage/electrodesiccation, 7.4-8.7\% for radiotherapy and $7.7 \%$ for cryosurgery (Rowe,1989; Nordin,1997; Lindgren,1997; Avril,1997). For Mohs' micrographic surgery, the recurrence rate is lowest, approximately $1 \%$ (Mohs,1986; Mohs,1988; Julian,1997). Actually, the effectiveness of a specific therapy should be defined on the results of evidence based research in cancer therapy: large, prospective (randomized, comparative) studies with long term follow-up ( $>5$ years), investigating the recurrence rates of a treatment modality (Bigby M, 1998). 
Surgical excision of isolated tumours, performed by experienced surgeons, will usually result in hardly visible scars, without impairing the functional neuromuscular aspects in the face. Because patients more and more develop multiple BCCs in the face at younger age, complete removal of the tumour is no longer the only important aspect of the treatment. In case of multiple facial tumours, at a certain point surgical intervention will become difficult because the amount of healthy skin left for closure of the defects after excision of the tumour will decrease, subsequently resulting in a serious risk for loss of functionality. For the future, there will be an increasing demand for non-surgical treatment modalities. Physical therapies like cryosurgery and radiotherapy cause destruction of both tumour tissue and a considerable amount of healthy surrounding tissue. Depending on the localization of the tumour, this will result in inferior cosmetic results, immediately or several years after treatment.

Photodynamic therapy (PDT) is a promising non-surgical treatment modality of increasing interest, because of its selective way of action on tumour tissue. PDT is an effective therapy for BCCs of the superficial subtype, but for the treatment of thicker, nodular BCCs the technique should be improved. PDT is a simple therapy to perform, but just this simplicity implies a certain risk to use this technique in the wrong way, for the wrong indications. 


\section{AMMS OF THIS THESIS}

In chapter 1 the epidemiologic aspects of $\mathrm{BCC}$ are reviewed to give an idea of of the "BCC problem". Subsequently, the principles and practical aspects of the treatment modilities surgical techniques, cryosurgery and photodynamic therapy are described in chapter 2.

The aim of the further studies in this thesis is dual. First, the final outcome of this thesis may contribute to the formulation of recommendations (or even the development of guidelines for the Netherlands) for diagnostic procedures, treatment and follow-up of the uncomplicated BCC. These recommendations will be based upon the results obtained from:

- A systematic review of the literature for 5-year recurrence rates after treatment of primary BCC for several current treatment modalities (chapter 3)

- A randomized, comparitive study for the cosmetic results after conventional surgical excision and cryosurgery for primary $B C C$ in the head/ neck area (chapter 4)

- An inquiry among the dermatologists in the Netherlands, to obtain information about their attitude towards making the diagnosis of $\mathrm{BCC}$, treating this tumour and performing tumour follow-up and preventional activities (chapter 5)

The second aim of this thesis is the investigation for improvement of the effectiveness of photodynamic therapy as a non-surgical, selective therapy for treatment of nodular growing BCCs. Both the penetration of the light and the penetration of the locally administered photosensitizer may be the limiting factors for effectiveness of PDT in thicker tumours. Two modifications of the technique for administration of the sensitizer are investigated with the intention to obtain a deeper localization of this sensitizer:

- A preceding partial debulking of the tumour mass on the final effect of PDT with a topically applied photosensitizer is investigated histopathologically in chapter 6 .

- In collaboration with the department of Pharmacology, Catharina Hospital, Eindhoven (A.W.de Blois, dr. R.J. Grouls and E.W.Ackermann), a solution for intra-tumoral injection of the photosensitizer was developed and is investigated in vivo for toxicity and dose-response relationship in chapter 7 . 
The effect of PDT may also depend on the illumination schemes.

- The influence of several combinations of illuminations on PDT-induced skin damage is investigated in chapter 8.

The different parts in this thesis are based on studies published in national and international journals or submitted for publication. Several parts of these studies have been re-written to make this book better readable as a whole, avoiding duplicate publications. For this reason also, the references have been listed separately at the end in the "bibliography". 
Chapter One

THE EPIDEMIOLOGY

OF

BASAL CELL CARCINOMA 
$\mathrm{BCC}$ is the most common cutaneous malignancy in Caucasians. This chapter deals with epidemiologic data concerning the registration and the incidence rates of $\mathrm{BCC}$ in the Netherlands, Northern/Western-Europe and the rest of the world. Subsequently, the exogenous and endogenous risk factors for developing BCCs are reviewed. Finally, the benefit of prevention and screenings campaigns will be discussed.

The data for this review were obtained by searching the several electronic databases (Medline, U.S. National Library of Medicine; Embase, Elsevier Science BV; Cancerlit, U.S. National Library of Medicine) for available information on these subjects.

\section{REGISTRATION OF NON-MELANOMA SKIN CANCER}

Accurate figures on the exact incidence of non-melanoma skin cancer (NMSC), and specific on BCC, are difficult to obtain, because most countries do not register them carefully. Besides the problems in building a cancer registration (Coebergh,1991; Schrijvers, 1994), the relatively favorable prognosis and the good therapeutic results seemed to make accurate registration less indicated in the past. On the other hand, many patients with $\mathrm{BCC}$ are treated in outpatient (private) clinics, without histopathologic verification of the clinical diagnosis. So, most of the incidence rates are just estimates, often based on surveys and samplings of selected areas of populations, which are not representative for the entire country. In general, there will be an underregistration of incidence rates, as stated in the cancer registrations in SW United Kingdom (Hughes, 1995), Scotland (Lucke,1997), Belgium (PierardFranchimont, 1999), the United States (Boring, 1994) and Australlia (Staples, 1998).

\section{INCIDENCE RATES OF BCC from a geographical view (Figure 1.1)}

All skin cancers together make up about a third of all the different types of cancer diagnosed in the world (Marks, 1995). NMSC predominantly occurs in the Caucasian population (Gloster, 1996). BCC represents $75 \%$ of all NMSC, and is therefore the most common malignant disease in Australia (Staples, 1998), the United States (Parker, 1996) and even throughout the world (Stern, 1999).

Based on incidence data presented by the Dutch Cancer Registration 
South-East, the estimated number of patients with new BCCs per year in the Netherlands (about 15 million inhabitants) was 18,000 in 1994, giving an incidence of 120 per 100,000 (Coebergh, 1995), with an expected increase by 2,700 new cases every year (Gezondheidsraad,1994). A survey conducted in 1996 among the dermatologists in the Netherlands showed that an estimated total number of approximately $30,000 \mathrm{BCCs}$ was diagnosed and treated annually (Thissen, 1998), suggesting that the increase in incidence was even more than expected. Compared with the period 1973-1977, the age-adjusted incidence rate for primary BCCs in the period 1988-1992 had increased by about $50 \%$ for males and almost doubled for females. Compared with other regions in Northern/Western Europe, the incidence rate for $\mathrm{BCC}$ in the South-East of the Netherlands was 25-50\% higher than in Denmark (higher latitude) (Osterlind, 1988), 15-30\% lower than in the Swiss canton of Vaud (higher altitude) (Levi,1988), and almost $60 \%$ lower than in South Wales (Celtic anchestry) (Lloyd Roberts, 1990). In Finland, the age-adjusted incidence rate was about 2.4 times as frequent from 1991 through 1995 (49 per 100,000 in men/45 per 100,000 in women) as from 1966 through 1970 (Hannuksela-Svahn,1999). Trends in the incidence of BCC in the Netherlands seem to fit into the pattern observed in other parts of Northern/Western Europe.

In Australia, where the incidence of $\mathrm{BCC}$ is highest, national random household surveys were conducted in 1985, 1990 and 1995 to estimate the NMSC incidence. In 1995, the age- and sex-standardized incidence rates of BCC were 788 per 100,000 , an increase of $19 \%$ since 1985 (Staples, 1998). Surveys among dermatologists in Geraldton even report incidence rates of 7 per 100 men and 3.3 per 100 women (English, 1997). The annual incidence in subtropical regions even approaches 2 per 100 among men and 1,5 per 100 among women (Green, 1996; Beuttner, 1998).

In 1995, an estimated 1.2 million of BCCs were diagnosed and treated in the United States (Miller,1994), where skin cancer is affecting one in every five persons (Rigel, 1996). The average annual incidence of BCC in the white population of the US ranged from 146 per 100,000 in Rochester, Minnesota (Chuang, 1990) in 1990, to 422 per 100,000 in Kauai, Hawaii (Reizner, 1993) in 1993. Incidence rates for BCC increased by more than $80 \%$ in both men and women between 1980 and 1994 (Karagas, 1999). For African-Americans on the other hand, the incidence rate for BCC is much lower (Halder, 1995) compared with Caucasians, but they are also increasing. 

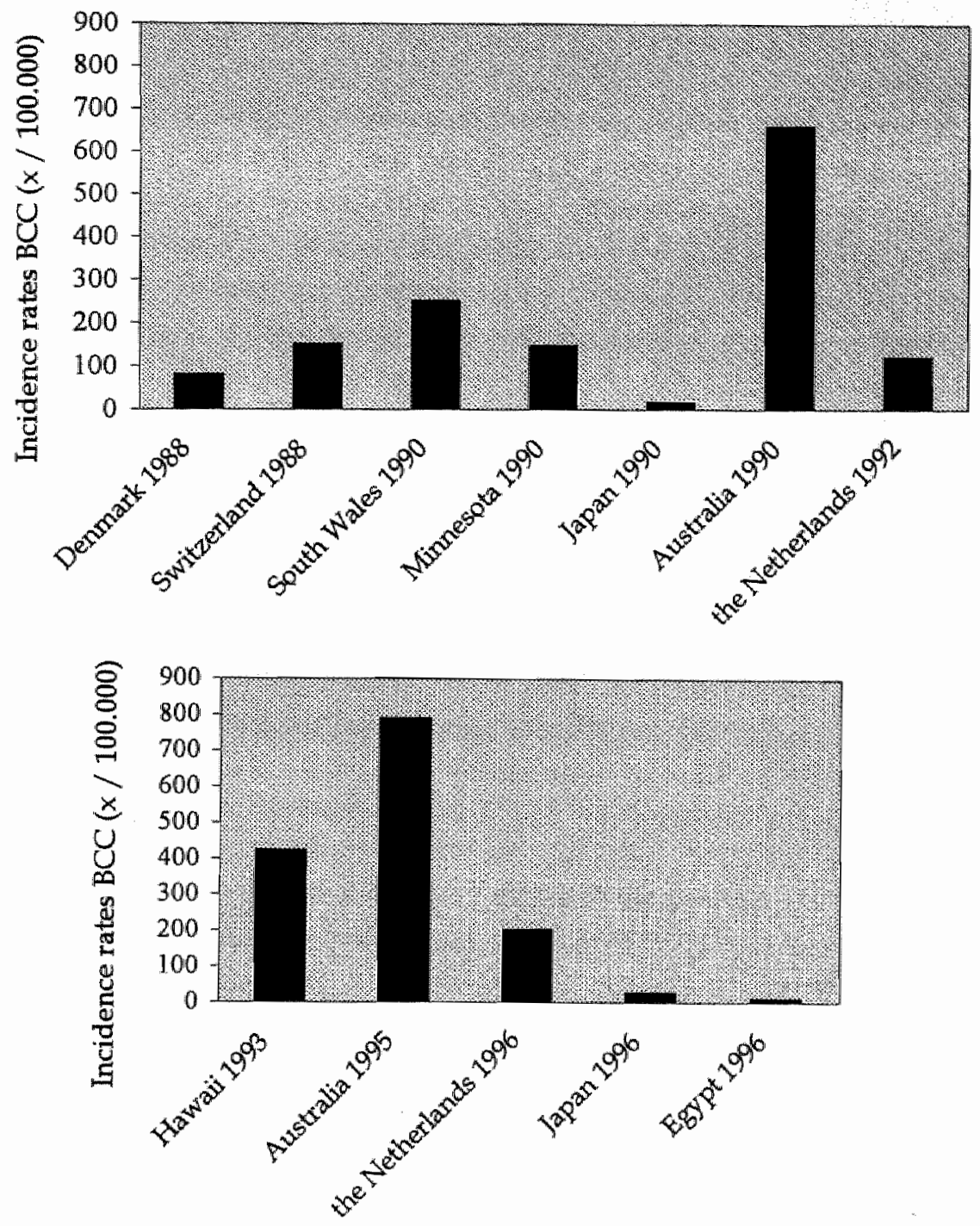

Figure 1.1

Incidence of BCC for the Local Population in Several Parts of the World for the Time periods 1988-1992 and 1993-1996.

An increase in incidence rate is demonstrated for Japan, Australia and the Netherlands. For all other countries, only the incidence rate of one time period is ayailable. 
Among the japanese people in Japan, the age-adjusted incidence rate was 16.5 per 100,000 in 1990 (Ichihashi, 1995) and 26.1 per 100,000 in 1996 (Nagano,1999). For the japanese population living in Kauai, Hawaii the annual incidence rate was 30 per 100,000 in 1987, suggesting that exposure to sunlight might be more important in the development of $\mathrm{BCC}$ than ethnic origin is (Chuang, 1995).

Although BCC is the most common malignancy in Caucasians, it rarely occurs in people with darker skin types. Among the black people in Nigeria, for example, BCC comprises only $2 \%$ of all skin cancers (Yakubu, 1995). The same situation can be observed in Indians where skin cancer accounts for 1-2\% of malignancies, with squamous cell carcinoma (SCC) representing 60-65\% of the skin tumours (Dhir, 1995). On the other hand, in Africa the incidence rates increase for persons with less pigmentation, even in geographical regions localized on higher latitudes. In Qatar (Egypt), for example, the average annual incidence per 100,000 was 9.9 for both sexes and at all ages (Mahmoud, 1996).

\section{MORTALITY AND MORBIDITY}

Despite the rising incidence of $\mathrm{NMSC}_{\text {, mortality rates are relatively low }}$ and have decreased over the last decade, possibly due to earlier and more effective treatment. BCC contributes much less tumour-related deaths than $\mathrm{SCC}$, accounting for $25 \%$ of the deaths (Dunn, 1965). In the Netherlands, mortality rates due to $\mathrm{BCC}$ are not available. In general, mortality seems to be greater in men than in women and also seems to be greater in white than in black people (Weinstock, 1993).

On the other hand, BCC may grow extremely aggressively. Because most BCCs are localized on the head and neck, this may result in significant morbidity with disfigurement, invasion of vital structures and mutilating local tissue destruction. Many authors described the phenomenon of so called "high risk" tumours. BCCs with any of the following characteristics: long duration, location in the H-zone (Figure 1.2) of the face and on parts of the nose, larger than $2 \mathrm{~cm}$, with agressive histologic subtypes (morpheaform, adenoid and micronodular), recurrent tumours or a history of radiation exposure, contribute to this group (Sahl,1995; Betti,1995; Randle,1996). Metastatic $\mathrm{BCC}$ is rare, varying between 0.0028 and $0.1 \%$ (von Domarus, 1984). Whenever this occurs, either lymphogenically or hematogenically to regional lymph nodes $(68 \%)$, the lung $(20 \%)$, the liver $(18 \%)$ or the skeleton $(17 \%)$, the 
prognosis is bad (mean survival period of 8 months). Metastasis is frequently associated with large-sized BCC (more than $5 \mathrm{~cm}$ in diameter) of any histologic subtype, or with irradical treatment in the past (Borel,1973; von Domarus, 1984; Lo, 1991; Randle, 1993; Schuller, 1997).

Figure 1.2

The H-zone, danger zone of the Face. This zone comprises the upper lip, the nose, the periorbital area and the lateral parts of the face, including the area around the ear.

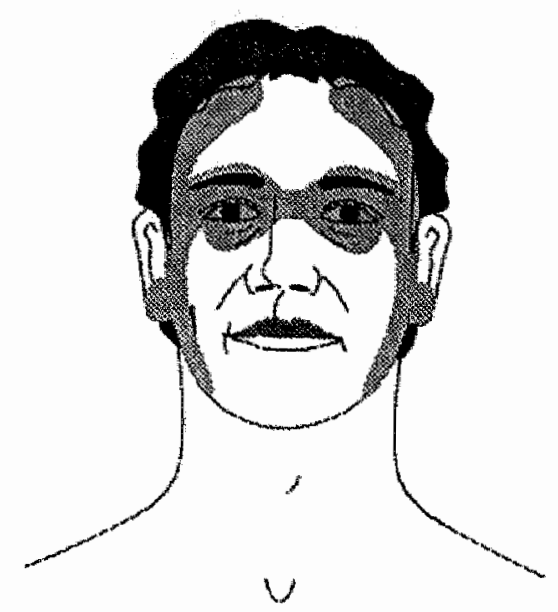

\section{RISK FACTORS: environmental factors and predisposing conditions}

The increased risk for development of a $\mathrm{BCC}$ is associated with several external and endogenous, patient dependent factors (Table 1.1), acting synergistically on the principal cause of $\mathrm{BCC}$ : intermittend, excessive exposure to UV-radiation, especially in children and young adults (Kricker, 1995a). Thus, most BCCs occur on parts of the skin excessively exposed to sunlight, like the head and neck (70\%), and in the last few years also on the trunk (20\%) (Bastiaens, 1998). The latter might be the result of more UV-exposure on the rest of the body during holidays and freetime spending. Further the incidence of BCC seems to increase with proximity to the equator (McKnight, 1979; Stone,1983; Ramani, 1993) where UV-dosage per unit light exposure is highest, and probably also with the total cumulative exposure to sunlight, as seen frequently in outdoor workers (Kricker,1995b) and in elderly persons (Kaldor, 1993). It has been suggested, that chronic UV-exposure will cause mutations 
Table 1.1

\section{Factors Associated With an Increased Risk of Developing Basal Cell Carclnoma.}

\begin{tabular}{|c|c|}
\hline 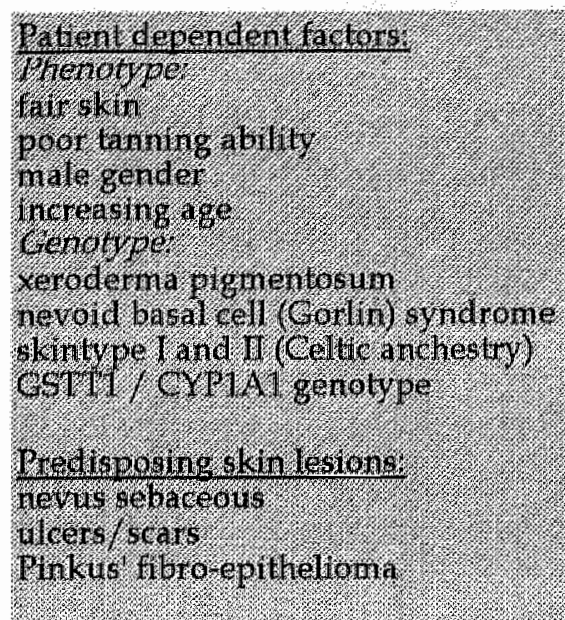 & 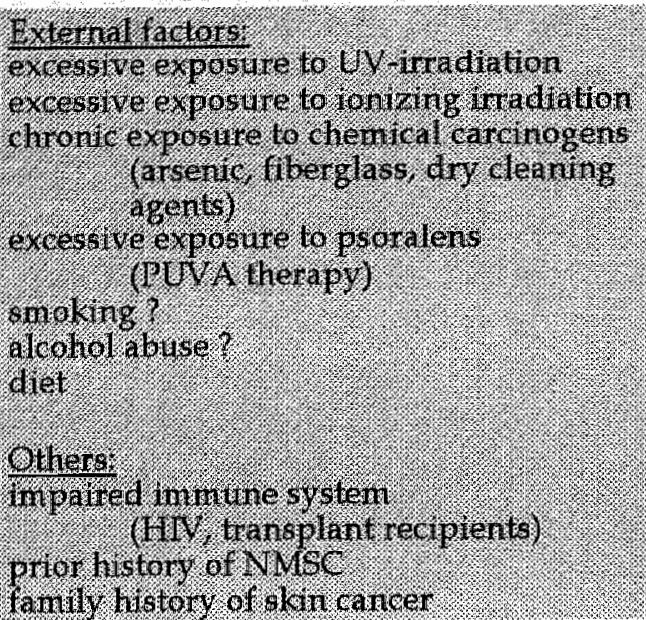 \\
\hline
\end{tabular}

in the p53 tumour suppressor gene. This gene normally plays an important role in the cellular response to DNA damage, either by causing a delay of the cell cycle providing additional time for the DNA repair, or by inducing programmed cell death (apoptosis) leading to the elimination of damaged cells. A failure in the apoptotic response after genomic damage could facilitate tumour development, as for BCC (Wikonkal, 1997; D'Errico, 1997). Additional factors to UV-exposure, which increase the risk for BCC are patient dependent phenotypical factors like fair skin (Green,1990; Lock-Andersen, 1999), poor tanning ability (Zanetti, 1996) and male sex (Chuang, 1990).

Other external factors associated with an increased incidence of $\mathrm{BCC}$, are exposure to high doses of ionizing radiation (treatment for skin disease/ cancer therapy, job-related or atomic bomb survivors) (Davis, 1989; Ron, 1998), chronic exposure to chemicals like arsenic (Shannon,1989), fiberglass dust and dry cleaning agents (Gallagher, 1996). Excessive exposure to psoralen during PUVA-therapy will also result in an increased risk for NMSC, mainly for SCC, but also for BCC (Lindelof, 1991).

The relation between some lifestyle characteristics and $\mathrm{BCC}$ has been investigated, resulting in a positive, but not statistically significant correlation for smoking and alcohol abuse. On the other hand, high doses of vita- 
mins $A, C$ and beta carotene and selenium may decrease the risk for developing BCC (Kune,1992; Sahl,1995).

Elevated risk for skin cancer has also been reported for persons who are immuno-compromised. Kidney and heart transplant recipients, taking immunosuppressive medication show a significantly increased risk for developing cutaneous malignant epithelial tumours, mainly of the SCC type, but also of the BCC type (Euvrard,1995; Hiesse,1997). An excess of BCC is also seen in patients with the acquired-immuno-deficiency-syndrome (Franceschi, 1998).

BCCs normally develop "de novo" and do not originate from precancerous skin lesions. However, there is one exception, concerning $0.5 \%$ of all BCCs arised from the premalignant fibro-epithelioma or Pinkus' tumour. On the other hand, there are a few predisposing skin lesions with an increased risk for developing a $\mathrm{BCC}$, like dermato-fibromas, the linear unilateral basal cell nevus and the nevus sebaceous (5-7\%) (Mehregan, 1965). BCC may also appear in scars and ulcers (Noodleman, 1986; Yang, 1996; Koga, 1997).

Except from the environmental factors, there are several genetic factors associated with an increased risk for BCC. Some years ago, evidence was found for the hypothesis that many patients develop skin cancers due to a genetically impaired repair of DNA, damaged by UV-radiation (Wei,1994; Grossman, 1995) as was demonstrated in patients with xeroderma pigmentosum and the nevoid basal cell carcinoma (Goltz-Gorlin) syndrome (Gorlin,1995; Kimonis, 1997). Other genodermatoses, associated with an increased risk for $\mathrm{BCC}$ are Bazex syndrome, Rombo syndrome and albinism (Michaelsson, 1981; Plosila, 1981). Recently, two families with multiple hereditary infundibulocystic basal cell carcinomas were described, suggesting the existence of another distinctive genodermatosis, different from multiple hereditary tricho-epitheliomas and Goltz-Gorlin syndrome (Luis, 1999). Although UV-exposure is critical, the development of multiple tumours in one person on sites less exposed to sunlight also suggests a genetic predisposition. This is confirmed by the finding of a significant interaction between typical loci encoding the detoxifying enzymes gluthatione S-transferase and cytochrome P450 (GSTT1 and CYP-1A1 genotypes) and multiple BCCs on the trunk (Lear,1997) .

\section{PREVENTION; public education and screening campaigns}

$\mathrm{BCC}$ has a high chance for cure if detected in an early phase of development, in which treatment can be performed in an outpatient setting with a minimum of discomfort, inconvenience and cost. High morbidity is 
frequently related to patient delay in seeking medical care. This delay is attributed mostly to lack of knowledge, rather than to fear and denial. In the Netherlands, as well as in many other countries therefore, primary prevention of skin cancer has focused mainly on convincing people that excessive UV-exposure, either natural or artificial (sunbeds), is unhealthy (Rhodes, 1995; Dobbinson, 1998). In the second place, people are informed how to prevent heavy sun-exposure; avoiding excessive exposure to sunlight between 10.00 am and $16.00 \mathrm{pm}$ ( $60 \%$ reduction of UVB radiation), using effective sunprotection creams (SPF) $>15$ for both UVA and UVB, every 2 to 3 hours; over $90 \%$ reduction of UVB), and wearing protective tightly woven clothing, sun glasses and hats. Primary prevention is especially important for infants and children, because excessive sun exposure during the early years of life appears to increase the risk for skin cancer, and thus for BCC (Marks, 1990; MMWR, 1997].

In the United States and in Australia, secondary prevention (early detection of the tumour) consisting of yearly national campaigns to promote skin awareness, self-examination and participation in free examinations to detect developing tumours, is sponsored by the dermatologic and cancer societies. The main problems of these screening programmes are the ongoing need for evaluating their effectiveness, and the barriers to obtain definitive diagnoses and treatments among screening-positive persons. Both compliance and follow-up will never be $100 \%$ and will depend on a patient's age (older patients seem to be more compliant than younger), a positive family history of skin cancer and additional cancer lesions (Jonna, 1998). In the Netherlands, screening campaigns so far have only been performed for cutaneous melanoma. The question remains whether these screening programmes are really valuable. The major objective of (volunteer) screening is: reducing mortality and morbidity, by finding disease and starting effective treatment at an earlier stage (Rampen, 1992). Although BCC is an important health problem, the benefit of screening for this indication is doubtful, because the problems which may result from these skin tumours are totally different from the problems arising from other cancers, like melanomas for example, and therefore they need their own approach.

For this moment, the effectiveness of the prevention efforts on the incidence of $\mathrm{BCC}$ can not be evaluated, because the time between UV-induced cell damage and the appearence of the tumour is longer than the period in which prevention campaigns have been performed. The first effect might be a 
decrease in the incidence rate among young adults, gradually moving to the older aged (Staples, 1998; Marks, 1999).

\section{CONCLUSIONS}

The incidence rates of $\mathrm{BCC}$ today are higher than they have ever been before, although these rates are not based on a thorough registration of NMSC. Taking the geographical situation into account; it seems that the incidence rate of $\mathrm{BCC}$ in the Netherlands is following the increasing trend in other European countries and in the rest of the world. The increase is mainly associated with increasing average age and several external and genetically predisposing conditions, all acting synergistically on the principal cause, excessive exposure to UV-radiation. Skin cancer prevention should be based on educational procedures (campaigns, instructions by physicians) aimed at avoiding excessive exposure to sunlight and promoting the use of protecting sun-screens and clothing, especially during childhood and adolescence. Patients also must be informed of the benefit of self-awareness. However, the benefit of (volunteer) sceening campaigns for BCC (and other NMSC) is doubtful in our eyes. 


\section{Chapter Two}

TREATMENT OF BASAL CELL CARCINOMA:

A Light thrown upon Surgical Intervention, Cryosurgery and Photodynamic Therapy

M.R.T.M. Thissen

H.A.M. Neumann

Submitted 
For the treatment of BCC several therapeutic modalities are available. Optimal treatment results, cure and cosmetic results can be achieved when the dermatologist is experienced in these different treatment modalities. Most uncomplicated BCCs are slowly growing tumours (Kirkup, 1999) of which almost 90 percent can be treated in general by standard office based surgical methods or physical treatments (Rowe, 1989) without developing recurrences during the first five years after therapy. The patient's age, general health, skin color together with the localization, size and histologic subtype of the tumour and history of previous treatments, if there were any, are important in determining the most appropriate treatment modality in an individual patient (Lang,1996; Marghoob,1997). In the second place, the cosmetic results, the costs, the after-care and possible complications, and the recurrence rates for each therapy must be evaluated for each patient individually.

Based on the 5-year recurrence rates derived from prospective studies in the literature (see Chapter 3; Thissen,1999), for the treatment of BCCs the choice should be made out of three standard therapies including primary surgical excision, Mohs' micrographic surgery and cryosurgery. All the other treatments should be considered as treatment modalities of second or third choice because of the higher recurrence rates or inferior long-term cosmetic results. Radiotherapy was very popular in the pre- and post-World War II years. The increase in incidence of $\mathrm{BCC}$, more patients with multiple $\mathrm{BCCs}$, and the first tumour at younger age in combination with knowledge of late complications (inferior cosmetic results, radiodermatitis and development of malignancies and the aggravating aspect for the patient changed the vision on radiotherapy among most dermatologists (Caccialanza, 1999). Today it is performed only in the elderly with BCCs outside the central part of the face and/or contra-indications for surgery. Curettage/electrodesiccation seems to be an inferior treatment, because it is a non-standardized technique and cure rates depend too much on the experience of the practitioner (Kopf, 1977).

Besides the conventional treatment modalities mentioned above, some other therapeutic options have been used. In the past, the effect of intralesional injections of interferon on BCCs has been evaluated, but the results were less favorable (Greenway, 1986; Buechner,1991). Topical treatment with 5fuorouracil or retinoids was shown to be effective in BCCs of the superficial 
subtype only (Stange, 1992; Brenner, 1993). Systemic retinoids are not effective for the cure of $\mathrm{BCCs}$, but they might diminish the risk for development of new tumours (Hughes, 1988). Also carbon dioxide lasers have been used for treatment of $\mathrm{BCC}$, but the results differ widely (Bandieramonte, 1997; Humphreys, 1998). At this moment, there are still new therapies under investigation, like the topical treatment with the cytokine- and interferon-inducer Imiquimod ${ }^{\mathrm{R}}$ (Beutner,1999) and topical application of substracts from the ajoen-plant which has cytotoxic effects on tumorigenic cells (Scharfenberg, 1990; Agarwal, 1996). Finally, the "old tool" photodynamic therapy (PDT) is revised for treatment of several cutaneous precancerous and malignant lesions and many investigators are trying to optimize this therapy to enlarge its effectiveness (Dougherty, 1998). However, it will still take many years before the cure rates of the investigational treatments will become available.

In this chapter the three standard treatment modalities for BCCs are desribed in detail: surgical excision, Mohs' micrographic surgery and cryosurgery. Photodynamic therapy, which seems to be a promising alternative, will be described in detail as one of the main subjects of this thesis. Initially some general remarks on safety margins and histopathological examination of diagnostic tumour biopsies and surgical excision specimens will be made.

\section{Safety margins}

Because most $\mathrm{BCCs}$ grow in continuity without producing satelite metastatic foci, it is only needed to treat the primary tumour mass to ensure its eradication. In order to do this, a margin of normal appearing skin around the visible border of the tumour is also treated (Breuninger, 1984); the wider the margin, the greater the possibility that the tumour is removed in toto. Safety margins, however, must be taken into account for any kind of treatment, not only for surgical excision. The principle of a safety margin of normal skin is based on the fact that we know that the tumour may extend less or more beyond its clinically visible borders, dependent on the histologic subtype (Breuninger, 1991). It is therefore important to assess the tumour type histopathologically and look for its diameter under optimal lighting, if necessary stretching and manipulating the surrounding skin to find the border.

For BCCs the safety margins have been calculated in relation to the confidence of entirely removing the tumour in $95 \%$ of the cases for both small, uncomplicated BCCs and larger "high risk" tumours (Breuninger,1984). The results are summarized in Table 2.1. In case of considering these mar- 
Table 2.1

\section{Relation Between the Clinical Tumour Diameter and Safety Margin for Surgical excision of Basal Cell Carcinoma (Neumann, 1996a).}

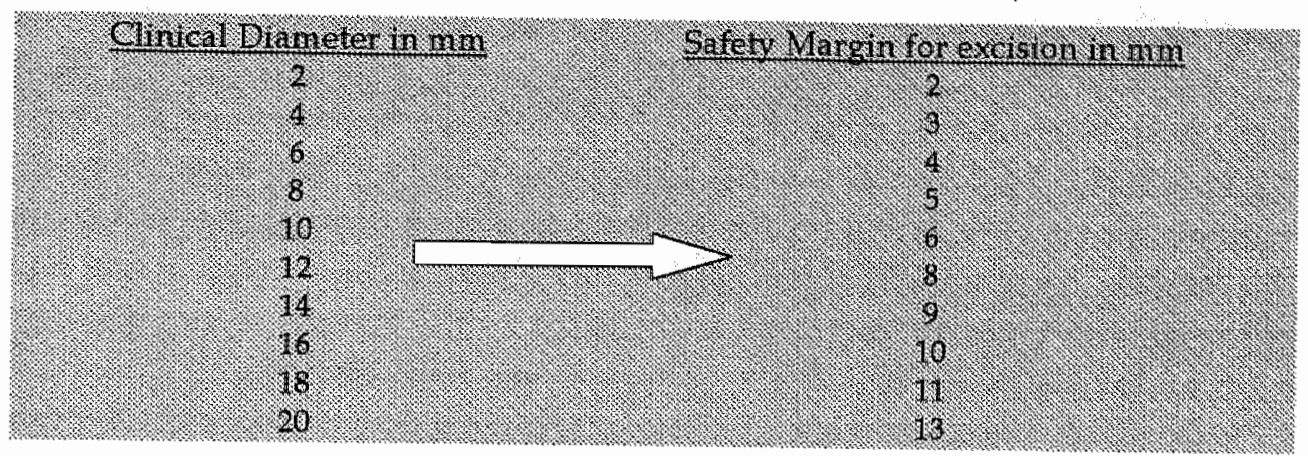

gins consistently, especially in the face this will result in unacceptably large defects. For this reason we recommend 3-mm margins for small $(<10 \mathrm{~mm})$ and 5-mm margins for larger $(10-20 \mathrm{~mm})$ nodular and superficial $\mathrm{BCCs}$ in the face and at least $5-\mathrm{mm}$ margins for nodular and superficial BCCs located elsewhere on the body (Neumann,1996a). Tumours with indistinct clinical borders (morpheic subtype), longstanding or recurrent tumours, and $\mathrm{BCCs}$ larger than $2 \mathrm{~cm}$ localized in the face are best treated by Mohs" micrographic surgery.

\section{Histopathological examination}

Both the diagnostic tumour biopsy specimen and the therapeutic surgically excised specimen should be fixed in formaline and submitted for histopathological examination. The tissue is routinely enclosed in paraffin and subsequently cut into vertical sections. Tumour cells in these sections become clearly visible after staining these sections routinely with haematoxylin-eosin (nuclues stains blue) (Elinitsas,1997). A time-saving and cheap, but less reliable alternative is staining with toluidine-blue. In difficult cases where it is doubtful whether the tumour is from epithelial origin or not, immunohistochemical staining with cytokeratin antibodies may give further information about the origin of the tumour. Also, if necessary, staining with Bcl-2 antibodies can differentiate between $\mathrm{BCC}$ and squamous cell carcinoma (Thissen,1997). In this way the histopathological growth pattern of a 
typical BCC can be obtained. The larger, surgically excised specimen should at least be marked with a suture for subsequent orientation, but even better for orientation is marking the bottom and all cutting-edges with different tissue dyes (Neumann, 1996b).

Although BCCs grow in a unifocal way and in continuity, many of them also grow with fine offshoots in several directions (Breuninger, 1992). These spurs can not be detected before treatment, not even by high-frequency Bscan (Breuninger, 1993). It will be clear that a complete 3-dimensional picture of the excision margins is required to evaluate whether the tumour really has been removed entirely. Therefore, with regard to the routine histopathological examination some critical remarks must be made. Because it is impossible for the pathologist to examine the entire specimen microscopically, the excised tissue is divided into a number of blocks. Subsequently the blocks are cut across their narrowest axis into several vertical sections. Using this "breadloaf" technique only $0,01 \%$ of the total cutting-edges can be examined (Figure 2.1) (Abide, 1984). So, one must realize that the positive predictive value of "margins contain tumour cells" is $100 \%$ true. But in cases where no tumour ceils are found in the section margins the conclusion that the tumour has been removed totally may not be drawn. Especially in "high risk" tumours different surgical and histopathological techniques are preferred like the "flounder" (Breuninger, 1994) and Mohs' micrographic surgery (Kopke, 1995). In both methods all lateral borders and the bottom of the specimen can be projected completely. Only the principles of cutting and flattening are different. Mohs' micrographic surgery is aimed at removing the tumour completely and saving as much healthy tissue as possible. By using the "flounder"technique the tumours including safety-margins of clinically healthy surrounding tissue (like in cases of standard surgical excision) are removed. Subsequently the central part of the excision specimen is removed, leaving a $2 \mathrm{~mm}$ thin layer of tissue at the borders and at the bottom. This part of the tissue can be projected in a three-dimensional way.

\section{CONVENTIONAL SURGICAL EXCISION}

\section{History}

The scalpel has been used for a long period of time to cut into a human body, both at the outside and the inside. Over the last decades, the surgical techniques have been refined by the development of qualitatively better surgical equipment, like different surgical blades, needle holders and materi- 


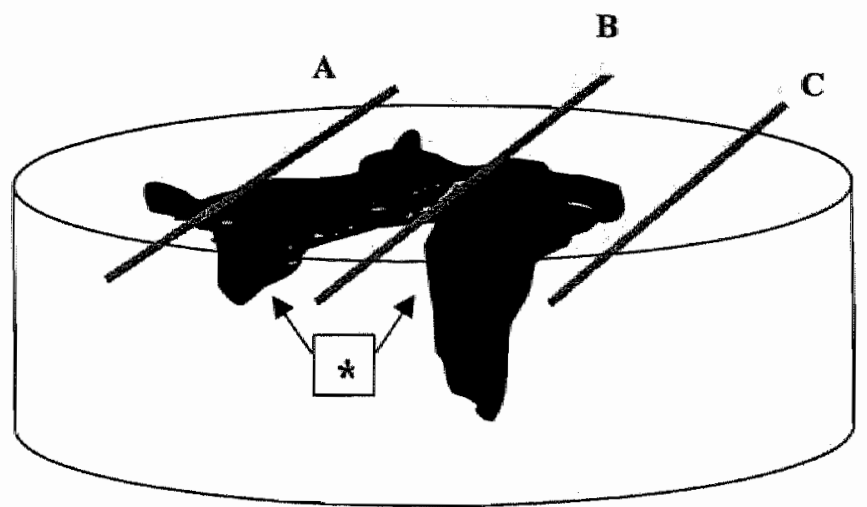

Figure 2.1

Schematic Reproduction of the Breadloaf Technique, demonstrating the Limitation of this Technique.

Routine histopathologic examination of the resection margins after surgical excision of $B C C$ usually will be performed using the breadloaf technique. At standardized distances, the excision specimen is cut into several vertical sections. In this way, tumour rests in the resection margins can be missed. In the sections $A, B$ and $C$, the cutting edges in the front are free of tumour cells, but in the non-examined parts (*) tumour cells are still present.

als for sutures. Medical practitioners also became more experienced in surgical techniques. These improvements, together with the fact that surgical excision is the only therapy that provides histological verification of radical treatment, have made surgical excision as the first-line therapy for treatment of basal cell carcinoma (Holt, 1994; Goldberg, 1997).

\section{Principles and practice}

In dermatology, surgical treatment can be performed under local anaesthesia by infiltrating the upper dermis with lidocaine or bupivacaine (Wildsmith,1984) with or without epinephrine (Backer,1980). In cases where the excision is planned in the skin area supplied by one great sensory nerve, a block of this nerve at its origin can also be performed. The excision should be done down into the subcutaneous fat, or the next uninvolved anatomical layer. The edges have to be undermined with blunt scissors to facilitate closure of the defect. Hemostasis should be performed carefully by electrocauterisation to prevent bleeding after surgery. Finally, the defect has to be closed either primarily by suturing the different (sub)cutaneous layers or by 
placing a splitskin or full thickness graft. Sutures in the face can be removed after 5-7 days, on the rest of the body after 10-14 days. If surgery is performed on the lower extremities, besides sutures, wound dressing with compressive bandages is recommended to prevent wound-dehiscence.

\section{MOHS' MICROGRAPHIC SURGERY}

\section{History}

The principles of Mohs' micrographic surgery date back to the 1930's. In this period, Dr. Frederick Mohs (assistant at the University of Wisconsin) performed experiments, injecting various irritants to tumours. He observed tumour necrosis after injecting a $20 \%$ zinc chloride solution, which had proved to posses anti-tumour properties (Caquoin,1913). On the other hand, microscopic examination showed a perfect preservation of the histologic appearance of this tumour, resembling a situation as if the tissue had been excised and fixated in formaldehyde. It was this kind of in-vivo fixation that layed the foundation of the development of the micrographic surgery according to Mohs, later called "Mohs' chemosurgery" (Mohs, 1978).

This technique, however, was just a means to achieve the final aim, complete histologic verification of tumour tissue. The disadvantage of this chemosurgery was the fact that only one single application followed by one single Mohs' cut could be performed, so it took several days for completion. The technique was also very painful for the patient and because the fixative was a foreign substance, an intense inflammatory reaction was evoked. In the 1960 's, the technique was modified, using local anesthesia and cutting fresh-tissue frozen sections (Tromovitch,1974). In this way the chemical fixative was no longer applied directly to the skin tumour and patients could be treated in one session. In 1985 the name of this technique was officially changed to "Mohs' Micrographic Surgery" (MMS): "micro" refers to "microscope", "graph" refers to a layer-by-layer illustration of the tumour configuration on a map that corresponds with the localization in the patient.

\section{Principles and practice}

Worldwide Mohs' micrographic surgery is the best technique to remove tumours combining maximal cure rate with minimal loss of healthy tissue. Also preservation of function and optimal cosmetic results are important factors (Mohs, 1978). The procedure begins with a precise drawing of the tumour on a Mohs'-map to ensure correct positioning during the operation, followed 
by careful assessment and marking of the clinical borders of the tumour. Subsequently, local anesthetic (lidocaine $1 \%$ with epinephrine, combined with a longlasting substance like bupivacaine $0.5 \%$ ) is injected by local infiltration, field blocks, and/or regional nerve blocks. After these preparations the tumour is debulked by curet or scalpel. Then the curetted wound including a small margin of epidermal layer is excised at an angle of $45^{\circ}$. The excised tissue is cut into small parts and the cutting edges are coloured to allow correct orientation of the removed tissue. Subsequently the excision specimens are frozen in the cryostat and the cut surfaces are carefully flattened during this freezing procedure. In this way it is possible to cut the whole resection margin (both deeper and epidermal layer) into horizontal sections (Figure 2.2). Accurate mapping of the excised numbered specimens is performed on the anatomical picture. By drawing marking-points both on the patient and in the picture a complete graphic reproduction of the excised tissue can be obtained (Picoto,1986; Motley, 1992; Miller, 1993). All the cutted sections are examined by both the pathologist and the Mohs" surgeon. Following the histopathological examination of the first cut, subsequential cuttings will be performed until all resection margins are free of tumour cells. After the tumour has been removed totally the decision can be made whether the final defect can be closed primary or by secundary healing.

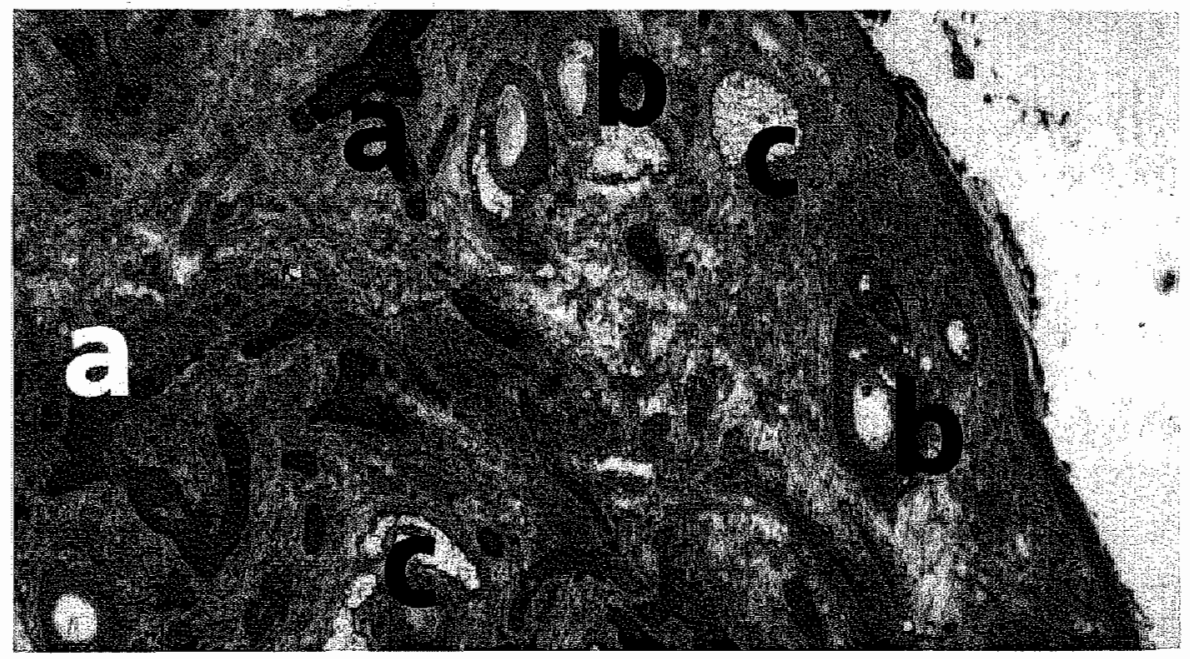

Figure 2.2

\section{Horizontal Section of BCC during Mohs' Micrographic Surgery}

Notice the strands of tumour (a) between the transverse cutting of the hairfollicles (b) and glands (c). In routine histologic examination following conventional excision, the appendages are cut in a longitudinal way. 
Mohs' micrographic surgery can be performed for almost all cutaneous malignant tumours, but it is especially indicated for the treatment of complicated $\mathrm{BCCs}$ localized in the face. Table 2.2 gives an overview of the BCCs that can be treated best by Mohs' micrographic surgery.

Table 2.2

\section{Indications for Mohs' Micrographic Surgery as First-Line Treatment of Basal Cell Carcinoma.}

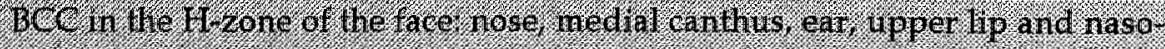
labial ford:

- Recinent BCC:

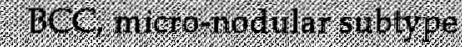

BCC morpheic subty :

In on pletely excised BCC.

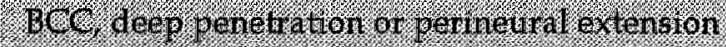

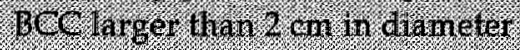

\section{CRYOSURGERY}

\section{History}

Cryosurgical techniques have been used since the second half of the nineteenth century. In 1851, James Arnott was the first to use the cryogenic temperatures of a cold saline solution to destroy tumours (Arnott, 1851). In later years other cryogenics like liquid oxygen, nitrogen, liquid air and carbon dioxide were developed. Liquid nitrogen was found to be the most potent cryogen (Rand,1968) and was initially applied by the swab method (Allington, 1950). In the 1960's, the neurosurgeon Cooper was the first to describe a closed system apparatus with cryoprobes utilizing liquid nitrogen (Cooper, 1963). By the development of these apparatus, the technique became more easy to standardize.

From that moment on, liquid nitrogen was adopted by dermatologists for the treatment of neoplastic lesions of the skin. In the following years, both Zacarian and Torre have made great effort to develop the dermatocryosurgical techniques resulting in the open-cone-spray method with neoprene cones as we still use it today (Torre, 1977; Zacarian, 1985). 


\section{Principles and practice}

Cryosurgery with liquid nitrogen can be an effective treatment modality for basal cell carcinomas if it is performed in the right way. The principles of cryobiology and the tissue reactions occurring after deep freezing must be taken into account (Zacarian,1985; Kuflik, 1990). The basic principle of cryosurgery is based on induction of selective necrosis by using cryogenic materials. Each freeze $\left(-5\right.$ to $\left.-50^{\circ} \mathrm{C}\right)$ and thaw cycle will be associated with a change in tissue texture or even a destruction of tissue. The most important injury factors may be listed as follows: (1) ice crystal formation both intracellularly and extracellularly, (2) disruption of cells by mechanical effects of ice crystal formation, (3) abnormal concentrations of electrolytes in the cell, causing $\mathrm{pH}$ changes, (4) cellular dehydration, causing cell shrinkage, (5) thermal shock with damage to cellular lipoproteins. Additional necrotizing effects will be caused by anoxia as a result of vascular damage.

The sensitivity for freezing is different for several cell types. Melanocytes are destroyed at higher temperatures $\left(-4\right.$ to $\left.-7^{\circ} \mathrm{C}\right)$ compared to keratinocytes $\left(-20^{\circ} \mathrm{C}\right)$. This explains the appearance of hypopigmentation after cryosurgery. Dermal connective tissue and fibroblasts can even withstand temperatures as low as $-30^{\circ} \mathrm{C}$. A temperature of $-50^{\circ} \mathrm{C}$ is necessary to obtain effective cryonecrosis in malignant tumours. Cartilage is totally unsusceptible for freezing, which makes cryosurgery an appropriate therapy for smaller BCCs on the eyelids (Fraunfelder, 1984) and parts of the ear (Zacarian, 1983).

The most severe injury is caused during rapid freezing procedures and slow thawing periods. In practice, two techniques of cryosurgery can be distinguished: the open-cone-spray technique and the closed-probe technique (Gage,1984). The first one provides faster freezing time and is thus more suitable for oncological application. In contrast to the swap method, cryosurgery using the special cryosurgery apparatus is a controlled technique. The length of the freezing period (and thus the final amount of tissue necrosis) depends both on the pressure used to get the liquid nitrogen out off the apparatus and on the diameter of the tip the nitrogen has to pass through. The technique can be standardized in three manners. The most difficult way requiring much experience, is by using thermocouples to determine the decline in temperature in the skin which is an indication for the cryogenic effect. Another way for control is to standardize the freezing and thawing times of the cryocycle (Zacarian,1985; Faber, 1986). A third, but less accurate method is the "measurement" in $\mathrm{mm}$ of the diameter of the freezing front, although 
there is no direct correlation between the diameter and the depth of the freezing front.

Cryosurgery is performed under local infiltration with lidocaine $1 \%$ with epinephrine. At first the lesion is thoroughly curetted with the intention to diminish the total tumour mass and also to explore the tumour borders (Spiller,1990). After curettage the remnants of the tumour are placed in an open neoprene cone taking into account a safety margin of $5 \mathrm{~mm}$ of normal skin. If the tumour exceeds $16 \mathrm{~mm}$ in diameter the field must be subdivided and each part must be treated separately. The best results will be obtained with a double freezing-thawing cycle (thawing time is at least three times the freezing time). A single cycle gives better cosmetic results, but will result in more recurrences. An additional third cycle will enlarge the effectiveness but will also lead to cosmetically unacceptable scars (Torre,1990). Freezing is performed best under the conditions listed in Table 2.3. Cryosurgery is relatively painless, except on localizations immediately above bony stuctures, like the pretibial area and on the back of the hands. The pain will diminish in several hours. Within 12 to 24 hours after cryosurgery a pronounced inflammatory reaction will appear, accompanied by a considerable edema.

Table 2.3

\section{Adjustment Cryosurgical Apparatus.}

Variabilut:

Ouflow pressure

(Tip ditaneter

THistance to sikin

Intermittenl / contiluous spray.

V Variation freezing / Hiawing crcle

- Movenient of the tip in the cone
Reconmendations for 4 diustinent

5. 8 psi (pound/ square meh)

$10.8 \mathrm{~min}$

tMaximu in 15 ain

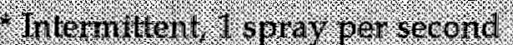

2. cyeles

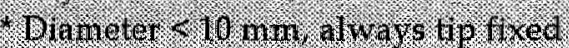

Table 2.4

\section{Advantages and Disadvantages of Cryosurgery.}

Advaniages:

Ouick

toutpatient treatinent:

trasy $1 \mathrm{~h}$ experienced hind:

* No adverso evenits in disorders of haen ostasis

Sures he ductus lace malis
Disadranitages

t I onglasting wound artet-eare

-No histologic verilteation afterwards

t IT popiginented stars

* Persistent tretaction in some lacations 
Table 2.5

Late Side-Effects of Cryosurgery.

t persistent hy popignentation.

4(Transieht) hyperpigmentation border

2 Atrophy / hyperthroply) central partscar (transient?)

- Millia along the border:

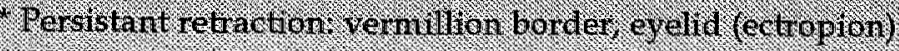

Alopecia

Table 2.6

Relative Contraindications for Cryosurgery.

Deperdient on Loction

Tlairy seals:

4 Exebrow

- Eyelfa boriter

* Vermilion border

TFacial danger zones (1)-zone)

T. Tow logs. feet
Ouler thimour related reasons

Diameter $>2 \mathrm{ch}$

- Recurrent BCC

TIXation to lone

Invasion in forniv conjunctiva

thvasion into subcutaneous tisstie

Afterwards necrosis will appear and healing of the wound may take several weeks.

Besides the time-saving and low-cost aspect of cryosurgery, there are also several disadvantages for this therapy (Table 2.4). Because melanocytes are very vulnarable to cryotherapy, the final result will be a permanent hypopigmented scar. Sometimes a (transient) hyperpigmented halo exists around the border of the scar. These discolorations make cryosurgery undesirable in darker skin types. Atrophy is seen frequently because the tumour usually has been completely destroying the normal skin. At some localizations retraction of the tissue may cause permanent functional disorders, like the ectropion after cryosurgery on the upper part of the cheek. Further late side effects are listed in Table 2.5. Infections, tendency to late bleeding or systemic reactions usually do not develop. On the other hand there is a minimal risk for damage to the lacrimal duct and superficially localized nerves, especially those running along the lateral sides of the fingers, the borders of the mandibula and the fossa ulnaris. Normally, damage of nerve fibres is transient and can be prevented by applying tumescent anesthesia. The relative contra-indications to cryosurgery are listed in Table 2.6. 


\section{PHOTODYNAMIC THERAPY}

\section{History}

The use of light as a therapy in medicine and surgery stretches back into antiquity. Phototherapy, also called heliotherapy, found its origin in ancient Greece, Egypt and India where it was used for treatment of vitiligo and psoriasis (Spikes, 1985). Photochemotherapy, or the use of an exogenous sensitizer to absorb light photons and than react for a therapeutic effect, has also a long history. Psoralens were already used in India in $1400 \mathrm{BC}$ (Fitzpatrick, 1959) and the well known PUVA-therapy (psoralen in combination with ultraviolet-A), as used for psoriasis nowadays, is based on the same principles. Thereafter both therapies disappeared for many centuries and it was at the beginning of the 20 th century that Raab and Finsen rediscovered them Raab, 1900; Finsen, 1901). Subsequently von Tappeiner took over Raab's research and published the first data of tumour regression using eosin as a photosensitizer (von Tappeiner, 1903). In 1904 he also reported that oxygen was absolutely required for photosensitization (von Tappeiner, 1904 and 1907) and he became the most important early pioneer of photodynamic therapy for treatment of malignancies. Meyer-Betz discovered the long lasting photosensitivity of the skin (erythema and oedema) after administering haematoporphyrin to himself followed by exposure to sunlight one day later (Meyer-Betz, 1913).

The actual development of PDT was initiated in the sixties by the work of Schwarz and Lipson (Lipson,1960; Schwarz,1955). A drug called haematoporphyrin derivative (HPD) was prepared by Schwarz during a study to explore the localizing properties of haematoporphyrin in neoplastic tissue. Lipson demonstrated that this substance could be used for diagnostic purposes (Lipson, 1961). After systemic administration HPD was localized in tumour tissue and could be detected by exciting HPD fluorescence with violet light. The main problem with HPD after systemic administration was the induced long lasting photosensitivity of the skin and the eyes for 6 to 8 weeks. It was in the late seventies that the development of new powerful laser lightsources lead to renewed interest in PDT. In 1975, Dougherty and co-workers showed complete eradication of mammary tumours in rats and mice after parenterally administered HPD activated by red light (Dougherty, 1975 and 1978). HPD was regarded as a first generation photosensitizer consisting of a mixture of porphyrins and their aggregates. The HPD drug Photofrin ${ }^{\text {TM }}$ has been registered officially in the Netherlands for treatment of bronchus carcinoma. Unfortunately, the specific localization of this drug is not limited to tumour 
tissue alone and the fluorescence yield is only weak. In the 1970's a worldwide experimental and clinical research on PDT started, searching for new photosensitizers with specific tumour localizing properties, absorption spectra of which the major peaks have wavelengths above $630 \mathrm{~nm}$ (the longer the wavelength, the deeper the penetration depth in the tissue) and less side effects (Roberts, 1989; v Leengoed, 1990; Szeimies, 1994 and 1995; Peng, 1997).

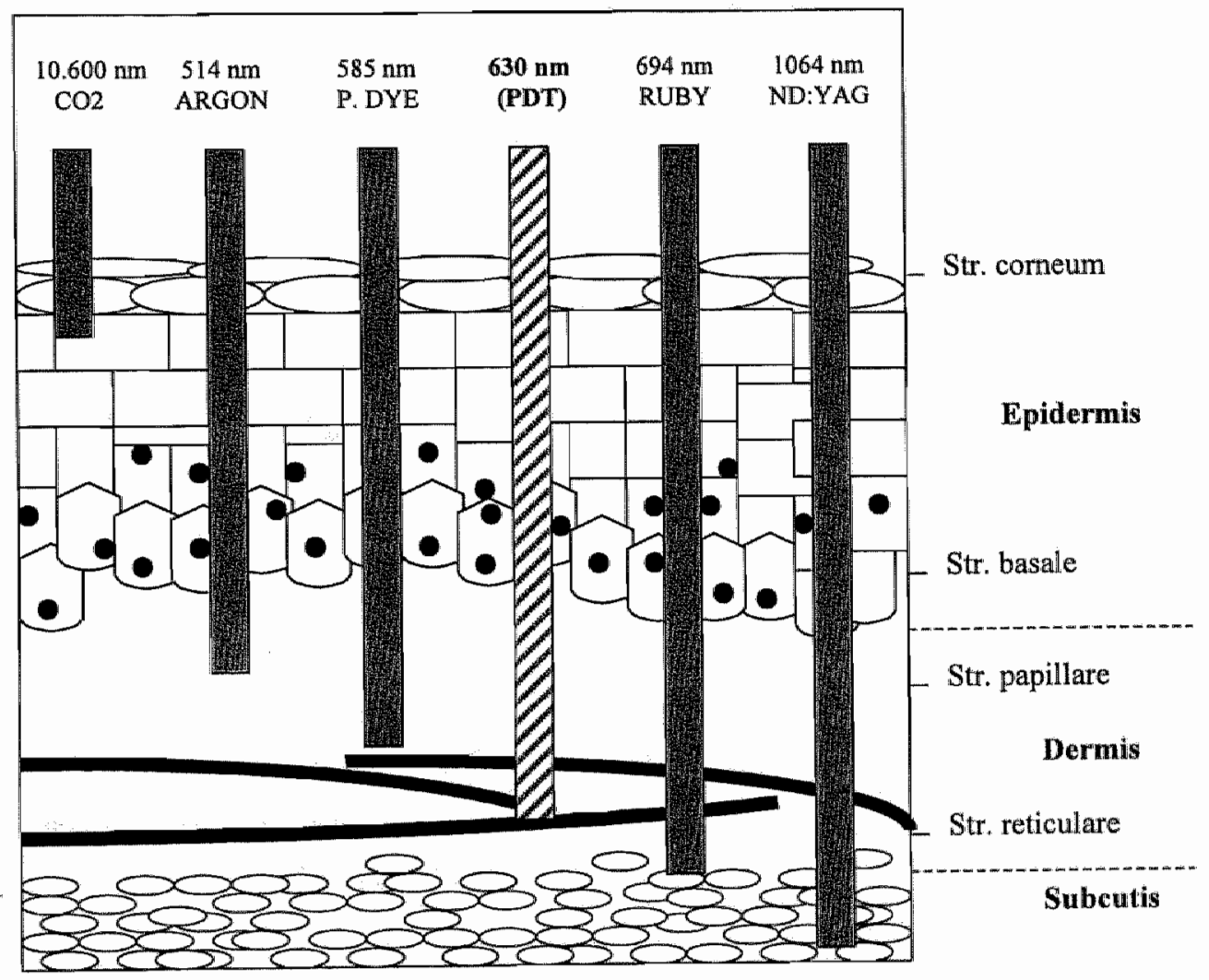

Figure 2.3

Schematic Representation of the Approximate Levels of Penetration into the Skin for Various Wavelengths.

Theoretically, light of $630 \mathrm{~nm}$, used for ALA-PDT will penetrate until the deep (reticular) dermis (redraun from Goldman MP. Cutaneous laser surgery; the art and science of selective photothermolysis. Mosby, St Louis, 1994). 


\section{Principles}

PDT is based on the dye-sensitized photo-oxidation of biological matter in the target tissue (Foote, 1990; Daniell,1991). One of the most important characteristics of a "good" sensitizer is the predeliction for localization and retention in malignant tissue. The mechanism of this predeliction is different for various sensitizers, but it is not completely understood (Peng, 1996; Fritsch, 1997; Langer, 1999). The dye (or sensitizer) is administered to the patient, either systemically or topicaliy, and after a certain time interval an optimum ratio between photosensitizer concentration in the tumour (high) and in the normal surrounding tissue (low) is reached. The tissue containing the sensitizer is subsequently exposed to light of a wavelength that corresponds with an absorption maximum of that sensitizer. The longer the wavelength, the deeper the light will penetrate into the tissue (Figure 2.3). Following the absorption of light, the sensitizer is involved in photochemical processes (Figure 2.4), leading to formation of cytotoxic oxidized products which will cause both direct and indirect (because of damage to vascular structures) cell death/necrosis or induction of apoptosis (Moan,1986/1990; Fingar,1992; Henderson, 1992; Roberts, 1994; Noodt,1996; Lavie,1999). PDT effects are evidently oxygen dependent which means that levels of tissue oxygenation play an important role in the final effectiveness of PD'T (Foster,1990). These photochemical processes are accompanied by fluorescence that can be measured on the surface of the skin. In this way, detection of fluorescence can be used as a diagnostic tool searching for tumour tissue.

In the past many fluorescent dyes have been investigated as photosensitizers for PDT. Systemic administration of porphyrins frequently resulted in longlasting hypersensitivity of the skin and eyes to light, depending on the type and the concentration used. Especially for use in dermatology, the development of a "third generation" photosensitizing substance, called 5aminolevulinic acid (5-ALA), was promising. 5-ALA is an exogenously administered precursor that stimulates normal and malignant tissue to produce its own endogenous photosensitizer. 5-ALA, normally present in all mammalian cells, is a precursor in the heme biosynthesis pathway and is converted into the final photosensitizer protoporphyrin IX (PpIX) (Figure 2.5). The biosynthesis of 5-ALA is regulated by a negative feedback control of ALAsynthetase by heme. Exogenous administration of 5-ALA bypasses this feedback control. In regenerating tissues and in various malignant tissues, the haem biosynthesis pathway could be disturbed because the porphobilinogen 
Fluorescence

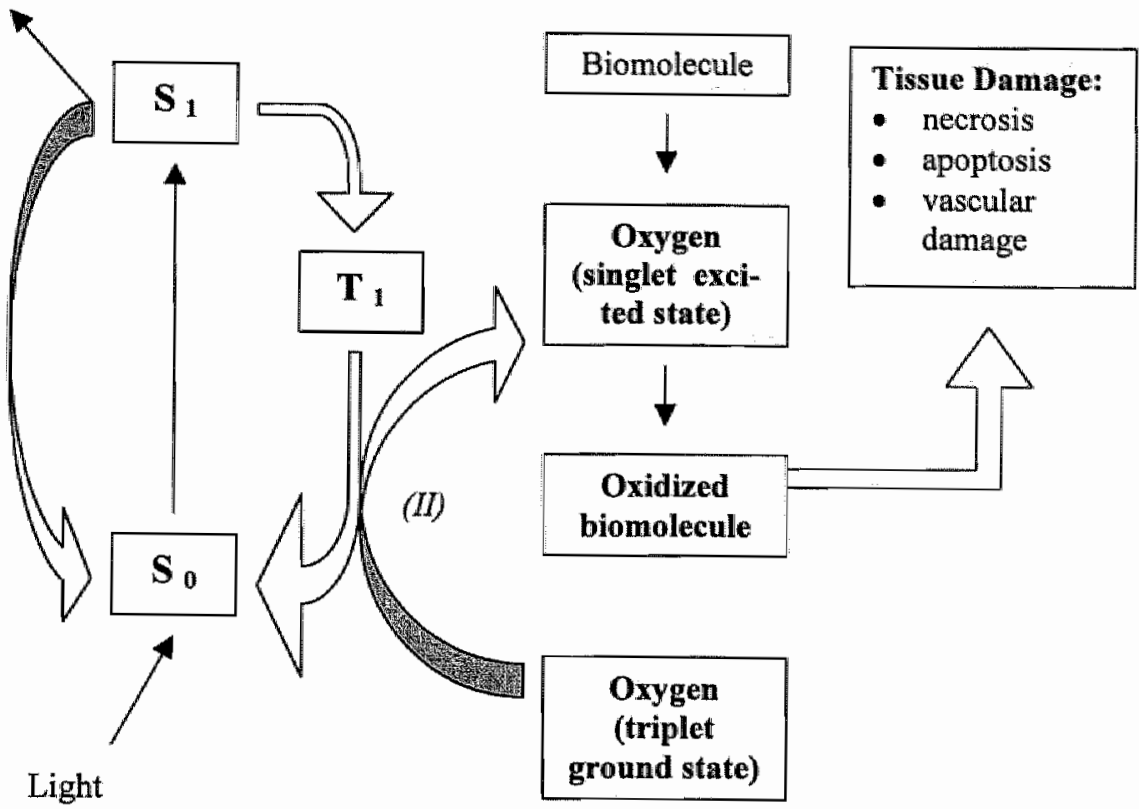

Figure 4.4

Simplified Scheme of Photosensitizer-Mediated Photochemical Processes Involved in Converting Photoenergy into Chemically Active Species in Photodynamic Therapy.

A photosensitizer molecule in the singlet ground state ( $\left.S_{0}\right)$ is excited to a higher energy state, e.g. to the singlet excited state $\left(S_{1}\right)$, by the absorption of a photon. Molecules at the short-lived $S_{1}$ state either decay back to the ground state (So) through fluorescence emission, or undergo intersystem crossing to the excited triplet state $\left(T_{1}\right)$. Molecules at the excited triplet state can undergo 2 types of reaction with surrounding molecules; an electron transfer process (type $I$ reaction; not in the figure) and an energy transfer process with an oxygen molecule (type II reaction), both proceeding simultaneously and in competition. The products of the type I reactions are free radicals, which can react with oxygen to give various oxidized products that initiate free radical chain reactions. In the type II reaction, molecules at the excited triplet state react with ground-state oxygen to produce excited singlet oxygen $\left({ }^{1} \mathrm{O}_{2}\right)$ and return the photosensitizer to the ground state (So). The ${ }^{1} \mathrm{O}_{2}$ species is highly active in biological systems, interacting with a number of proteins, lipids and other biological molecules. The ${ }^{1} \mathrm{O}_{2}$ species can also effect cell death, which is the most important factor in the PDT-induced tissue damage. 


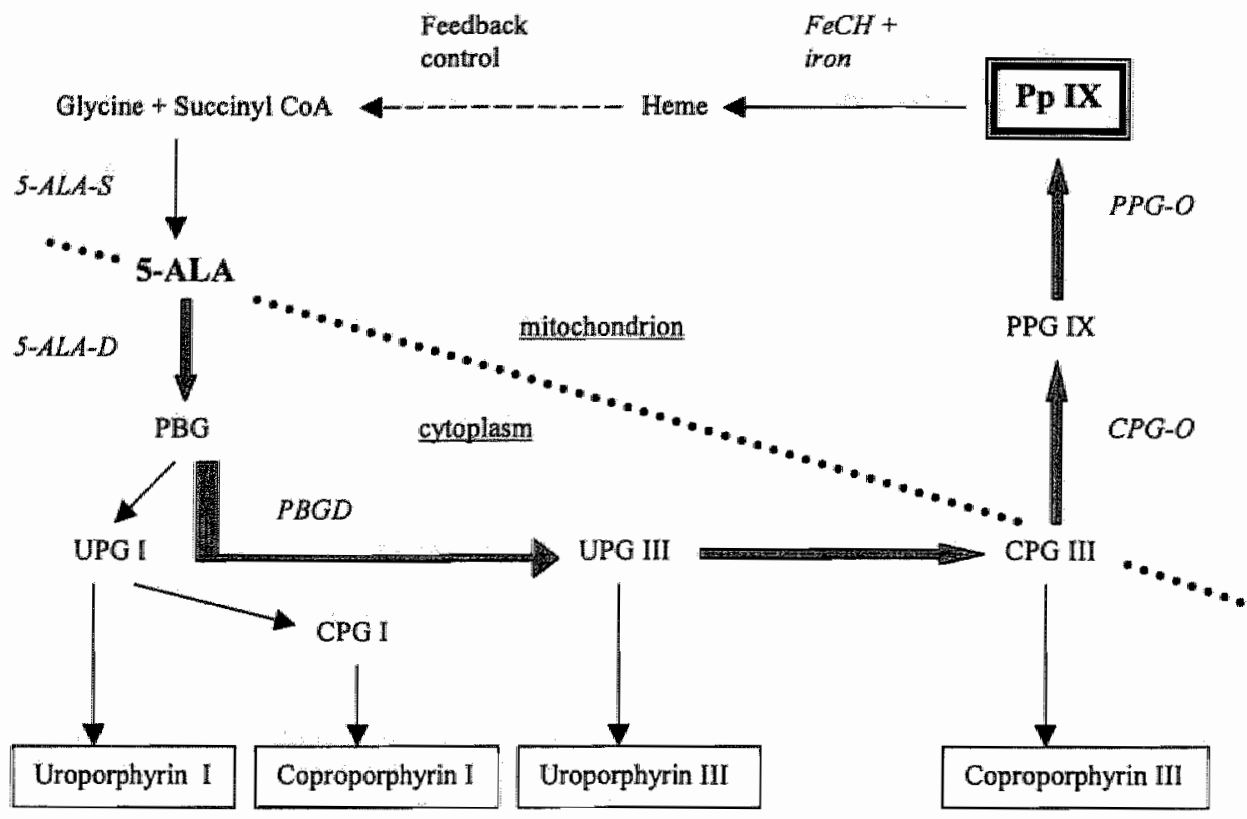

Figure 4.5

\section{Simplified Biosynthesis Pathway for Heme.}

The principal biosynthesis of aminolevulinic acid (ALA)-induced protoporphyrin $L X(P p L X)$ is indicated by open, grey arrows. The steps between conversion of 5-ALA via porphobilinogen (PBG) and uroporphyrinogen III (UPG III) into coproporphyrinogen III (CPG III), the second last precursor of protoporphyrin IX, take place in the cytoplasm of the cell. The final two conversions of CPG III into protoporphyrinogen. $I X$ (PPG LX) and successively PPG IX into PpIX take place in the cellular mitochondrial matrix. All fluorescent and photosensitizing components formed in this pathway are enclosed in rectangles, with $P p L X$, the most potent sensitizer, enclosed in the double-lined rectangle. The heme feedback control mechanism is indicated in the top with a dashed line.

(Abbreviations of important enzymes in the heme-pathway (italic in the figure): 5-ALA-S, 5-ALA synthase; 5-ALA-D, 5-ALA dehydrathase; PBGD, porphobilinogen deaminase; $C P G-O$, coproporphyrinogen oxidase; $P P G-O$, protoporphyrinogen oxidase; $\mathrm{FeCH}$, ferrochelatase). 
deaminase (PBGD) activity is often increased, whereas the ferrochelatase activity is decreased. As a result higher concentrations of PpIX will be present in neoplastic tissue for a longer time period compared to the situation in normal tissue. This phenomenon provides the basis for selective tissue damage (Schoenfeld, 1987 and 1988; v Hillegersberg, 1992; Fritsch, 1997).

Animal experiments have shown that, after intravenous administration of 5-ALA only certain types of tissue including the skin show PpIX fluorescence (Loh, 1993). 5-ALA is hydrophilic and is therefore, besides for systemic administration, well suited to be dissolved in a cream for topical administration, which makes 5-ALA suitable for PDT of cutaneous lesions. PpIX on the other hand is hydrophobic and lipophilic, has a high affinity for membranes and therefore, once synthesized cannot easily be removed from the cell (on the other hand, PpIX is metabolized). This means that the final PDT induced damage will be caused to these membranes (e.g. lysosomes, mitochondria and cytoplasm).

\section{Practice}

After application of 5-ALA in cream it takes 4 to 8 hours for the human cells to synthesize enough PpIX for effective PDT which means that illumination can only be performed with success after a time interval of at least four hours. PpIX is sufficiently present in the tumour cells for 12 to 16 hours which means that illumination after this period will be less effective. Superficial BCCs can be treated effectively with topical ALA-PDT. To improve the response to PDT of the thicker nodular $\mathrm{BCCs}$, a preceding superficial debulking of the tumour mass can be performed with the intention to obtain penetration of both the sensitizer and the light till the bottom of the tumour (Soler, 1999; Thissen, 2000).

PpIX can be excited by different wavelengths, corresponding with the absorption spectrum of this sensitizer. For therapeutic indications, light at a wavelength of $630 \mathrm{~nm}$ is used. The optimum illumination time, the total light dose and the illumination frequency are still under investigation (Fingar, 1987; van der Veen,1994; Pogue,1997; Robinson,1998). In the studies reporting on the results of PDT for BCCs generally single irradiations with total light doses between 50 and $150 \mathrm{~J} \mathrm{~cm}^{-2}$ and fluence rates between 50 and $100 \mathrm{~mW} \mathrm{~cm}^{-2}$ are used (Svanberg,1994; Wennberg, 1996). Immediately after PDT the treated area shows signs of a phototoxic reaction like oedema and erythema, finally resulting in necrosis of the tumour cells within 48 hours and formation of a 
scab. In the following days the skin will heal with excellent cosmetic results without atrophy, hypo- or hyperpigmentation. Protection against daylight, as required after systemic administration of Photofrin ${ }^{R}$, is not necessary.

Besides the therapeutic $630 \mathrm{~nm}$ wavelength, used for PDT, excitation can also be performed with $514,5 \mathrm{~nm}$. Light of this shorter wavelength will penetrate less deep than $630 \mathrm{~nm}$ light. Excitation, however, always leads to induction of the mechanism of PDT resulting in tissue damage. If fluorescence measurements are used for diagnostic purpose only, the induction of tissue damage is not needed and should even be prevented. In this case, excitation of PpIX with 514,5 nm can be used as a diagnostic tool for localizing tumour cells. Another, even more shorter, excitation wavelength for PpIX is $405 \mathrm{~nm}$. The main problem with this wavelength is the fact that several tissues, including the skin, show relatively high auto-fluorescence signals around $400 \mathrm{~nm}$, making the differentiation between this autofluorescence and PpIX fluorescence more difficult (van der Veen, 1999). 


\section{Chapter Three}

A SYSTEMATIC REVIEW OF TREATMENT MODALITIES FOR

\section{PRIMARY BASAL CELL CARCINOMAS}

M.R.T.M.Thissen

H.A.M. Neumann

L.J. Schouten 


\begin{abstract}
Objective: systematic review of the literature for studies reporting on the recurrence rates of $B C C$ after different therapies.

Design: all studies published in English, French, German, Dutch, Spanish or Italian between 1970 and 1997, that prospectively examine recurrence rates for at least 50 primary BCCs followed for at least 5 years after treatment with MMS, SE, CE, CS, RT, IM or PDT were reviewed.
\end{abstract}

Setting: the review was performed by the Department of dermatology in the Academic Hospital of Maastricht, the reference centre for dermato-oncology and MMS in the Netherlands.

Main Outcome Measure: comparing the recurrence rates after different therapies for BCCs, resulting in the development of guidelines for treatment of BCCs.

Results: 298 studies were found in several electronic databases. Only 18 studies met the requirements and could be used for analysis. MMS resulted in the lowest re. currence rates after 5 years, followed in increasing order by SE, CS and CE.

Conclusions: recurrence rates for different therapies could not be compared, because of lack of uniformity in the method of reporting, so evidence based guidelines could not be developed. We suggest that MMS should be mainiy used for larger, morpheic BCCs lokalized in danger zones. For smaller BCCs of the nodular and superficial type, surgical excision remains the treatment of first choice. Other treatment modalities can be used in cases where surgery is contra-indicated. Immunotherapy and photodynamic therapy are still investigative. 
Over the past 50 years, hundreds of articles about therapies for primary $\mathrm{BCC}$ have been published in literature. The most important aspects reported are the recurrence rates after conventional surgical excision (Goldberg, 1997), cryosurgery (Bullock, 1976; Zacarian, 1983), curettage/electrodesiccation (Edens, 1983; Adam, 1986), radiotherapy (Fitzpatrick, 1984; Caccialanza, 1999) and Mohs' micrographic surgery (Robinson, 1993); the treatment modalities most used. Unfortunately, in the literature the method of reporting the recurrence rates is not uniform. These rates mainly depend on the time for follow-up, varying from 6 months to more than 10 years (Rowe, 1989). Another difficult aspect is the heterogenity of the studies reporting on the results of several therapies, concerning typical characteristics of the tumour, like localization, size, histologic subtype, and the patient characteristics like age, sex and other medical problems (Leffel, 1991; Lang, 1996; McCormack, 1997; Marghoob, 1997) .

In this chapter, all prospective studies from 1970 to 1997 on treatment modalities for primary $\mathrm{BCC}$ are reviewed, to compare the effectiveness (recurrence rates) of the five most commonly used treatment modalities and two investigational treatment modalities, immunotherapy with interferon or 5-fluorouracil and photodynamic therapy. In addition, possible explanations for the variation and controversies among study results are given, with regard to study-design, statistical methods, and tumour and patient characteristics.

\section{METHODS}

\section{Treatment modalities}

Studies reporting recurrence rates after the treatment of primary $\mathrm{BCC}$ by Mohs' micrographic surgery (MMS), surgical excision (SE), cryosurgery (CS), curettage and electrodesiccation (CE), radiotherapy (RT), immunotherapy (IM) and photodynamic therapy (PDT) were reviewed. The validity of each study was examined and the data concerning recurrence rate were analyzed.

\section{Data search}

The Medline Standard computer database (U.S. National Library of Medicine), the Embase computer database (Elsevier Science BV), the Medline 
Advanced (Internet) database and the Cancerlit database (U.S. National Library of Medicine; also including unpublished articles and meeting abstracts) were searched for articles from 1970 to 1997. The following catch words (including analogues and derivatives) were used: 'basal cell carcinoma', 'basalioma' or 'epithelioma' in combination with '(surgical) excision', 'surgery', 'Mohs' (micrographic) surgery", 'cryotherapy', 'cryosurgery', 'electrodesiccation', 'curettage', 'radiation-therapy', 'radiotherapy', 'immunotherapy', 'interferon', '5-fluorouracil', 'photodynamic therapy', 'd/5-aminolevulinic acid' and "skin cancer". The 'Year books of dermatology" (1978 - 1996) were screened for studies manually. Finally, textbooks, reviews, editorials and existing guidelines, as well as the references of the studies found were checked for further information.

\section{Inclusion- and exclusion criteria}

Prospective studies reporting the recurrence rates after MMS, SE, CS, $\mathrm{CE}, \mathrm{RT}, \mathrm{IM}$ and PDT as treatment of all histologic subtypes - superficial, nodular, micronodular, adenoid and morpheic - of previousiy untreated basal cell carcinomas were included. The follow-up had to be for at least five years or more for each tumour treated. Studies published in English, French, German, Dutch, Italian and Spanish were selected.

The following criteria were used for excluding data (Table 3.1): studies reporting on cutaneous malignancies others than $\mathrm{BCC}$, as far as the results are not described separately for each cancer type; retrospective analysis; recurrent BCCs; follow-up shorter than 5 years; the results of less than 50 tumours and patients; the effectiveness of the therapy, as proved by excision and histopathologic examination several months after treatment; and cosmetic results only. Duplicate publications, reviews on certain therapies and reports on combinations of two or more therapies were also excluded.

\section{Study selection and Data extraction}

For each patient series included, the treatment modality, the number of $\mathrm{BCCs}$ treated, the recurrence rates and the duration of follow-up were recorded. The histologic subtype, the size and localization of the tumours, the number of tumours per patient and the number and reasons for dropouts were not analyzed. This provided an adequate number of studies to compare the different treatments. Studies were categorized in three groups, according to the number of tumours treated $(50<n<99,100<n<249$ and $n>250)$. 


\section{Data analysis}

If possible, the study size-weighted recurrence rates for all the groups of tumours were calculated by dividing the total number of recurrences by both total number of tumours treated (raw recurrence rate), and by total number of patients observed for at least 5 years (strict 5 year recurrence rate). The mean weighted recurrence rates for each treatment modality were also calculated 2 ways. Finally, life-table cumulative 5-year recurrence rates were either recorded from the articles or calculated in cases in which sufficient data for these calculations were available.

\section{RESULTS}

\section{Literature search}

In total, 298 studies, published between 1970 and 1997, were identified in the several databases. Of these, 153 were found in the Medline Standard database and an additional 70 in the Medline Advanced database (Internet), 28 in the Embase database and 47 in the Cancerlit database. A screening of reference lists, abstract books and yearbooks of dermatology revealed no additional studies. Fifty-one percent of all studies were found in the Medline Standard database, the database most frequently used all over the world. Only 18 of the 298 studies found were large, prospective studies of primary $\mathrm{BCC}$ with a follow-up longer than 5 years. Table 3.1 shows the criteria for exclusion for each treatment modality. Initially, 29 studies written in a language other than those mentioned previously, 25 duplicate publications and 13 reviews were excluded. Also excluded were 16 studies reporting on the histopathological verification of a treatment modality several months after therapy. Finally, 1 study reporting on the cosmetic results only and another 51 studies reporting on different criteria - comparison between 2 or more therapies $(n=16)$, etiologic studies $(n=9)$, a review of the treatment of $B C C$ in general $(n=7)$, a combination of 2 or more treatment modalities $(n=7)$, primarily wound care $(n=3)$, a meeting abstract without exact data $(n=2)$, follow-up after incomplete excision $(\mathrm{n}=2)$, a discussion of excision margins $(n=2)$, radiotherapy as adjuvant therapy $(n=2)$, and a case report $(n=1)$ - were excluded. After the main exclusion criteria (not confined to $\mathrm{BCCs}$, includes recurrent BCCs, retrospective studies, follow-up shorter than 5 years, and patient series $<50$ ) were applied to the 133 remaining studies, 18 patient series could be included. The main reasons for exclusion were a follow-up shorter than five years (59 out of $229 / 25,8 \%$ ), and that the studies were re- 
trospective ( 33 out of $229 / 14,4 \%$ ). In 22 patient series $(9,6 \%)$ no difference was made between the treatment of primary or recurrent BCCs and 16 series $(6,9 \%)$ reported on the results of the treatment of cutaneous tumours in general (BCC, squamous cell carcinomas and precancerous lesions). In another 15 series $(6,6 \%)$ the number of tumours treated was considered too small $(n<50)$.

The final total of 18 studies included reports on the treatment of 9930 primary BCCs (Table 3.2). Although some patients were observed for more than 5 years, the exact figures could not be calculated because several studies mentioned only the recurrence rates without the absolute number of patients BCCs observed for more than 5 years. Six patient series reported on 4212 tumours treated with $\mathrm{CE}$. Three reported on 1303 patients with BCCs treated with SE and another 3 reported on 2660 patients with BCCs treated with MMS. Four series reported on 798 patients with BCCs treated with CS and 1 series reported on 862 patients with BCCs treated with RT. Among the investigational treatment modalities, 1 series reporting on 95 patients with BCCs treated with immunotherapy was included. For PDT no patient series was included because the follow-up was too short in all studies.

The exclusion of a few studies will need some further explanation. The paper by Emmett, reporting on the results of SE on 2539 BCCs, was not included because the author described a surgical technique with wide resection margins $(<10 \mathrm{~mm})$ and frozen section analysis of the excised tissue (Emmett, 1990). If the free margins in the frozen paraffin sections were to small according to the histologic subtype of the $\mathrm{BCC}$, a re-excision was performed. This technique will absolutely decrease the risk on recurrence of a $\mathrm{BCC}$, and the results cannot be compared with those of surgical techniques reported on in other studies.

Also excluded from this review was the paper by Breuninger, reporting on the results of SE on $2016 \mathrm{BCCs}$ using the so called flounder technique (Breuninger,1989a). This implies a 3-dimensional histologic control of the excised tissue, evaluating some cross-sections, 2 additional edge sections and the undersurface (Breuninger, 1984a). The principles of this technique, and maybe the results, are comparable to Mohs' micrographic surgery with only one difference: MMS is aimed at removing all the cancerous tissue and saving as much healthy tissue as possible (Robinson,1993), whereas the flounder technique does not include the second aspect of saving healthy tissue. This might lead to less favorable cosmetic results. The flounder technique 
was developed and performed by Breuninger only, so the results of that study can not be compared with the others.

An impressive study by Dinehart et al. (1992), reporting on the difference in the presentation of $\mathrm{BCCs}$ and the results of MMS for $\mathrm{BCC}$ in younger patients compared with older patients, was not included because the follow-up was too short (median 4,2 and 4,4 years; range 5 months to 10,7 years).

Glatt et al. (1992) reported the results of SE in 236 primary and recurrent periocular BCCs, but these tumours were resected under conventional frozen section control, implying a lower recurrence rate than for "simple" SE. The study was excluded for that reason.

In the category RT, large studies like the one by Schneiter and Krebs (1982) and the one by Reymann and Kopf (1978) had to be excluded because the results were analyzed retrospectively. The studies by Lippert and Wiskemann (1978) and by Petrovich et al. (1987) failed to report the results of the primary $\mathrm{BCCs}$ separated from recurrent $\mathrm{BCCs}$ and were excluded.

\section{Effectiveness / Recurrence rates}

The results of the literature search are summarized in Table 3.3. Because most authors used different statistical methods for calculating the results of their therapies, it is impossible to give an overall mean recurrence rate for each treatment modality. The real recurrence rate will be somewhere between the estimated weighted recurrence rate after 5 years follow-up and the estimated weighted recurrence rate for all $\mathrm{BCCs}$ treated from the start of the study.

For MMS, the average weighted estimated recurrence rate lies between $0,8 \%$ $(0,5-1,3)$ and $1,1 \%(0,6-1,7)$. For CS, these values are $3,0 \%(0,0-3,3)$ and $4,3 \%(0,0-5,2)$, one study by Fraunfelder (BCCs on the eyelid, larger than 10 $\mathrm{mm})$ reporting on much higher recurrence rates $(11,4 \%$ and $20,4 \%)$ compared to others. For one single IM-study, the result lies between $12,6 \%$ and $21,4 \%$. For SE, CE and RT the average weighted estimated recurrence rates could not be calculated because of the lack of necessary data. For SE the average cumulative 5-year recurrence rate is $5,3 \%(3,2-8,0)$ and for one single RT study $7.4 \%$.

For CE neither an average weighted estimated recurrence rate nor an average cumulative 5-year recurrence rate could be calculated because of missing some important data; the weighted recurrence rates for all $\mathrm{BCCs}$ are varying between 3,8 and 7,7 percent, with a high value in a study performed 


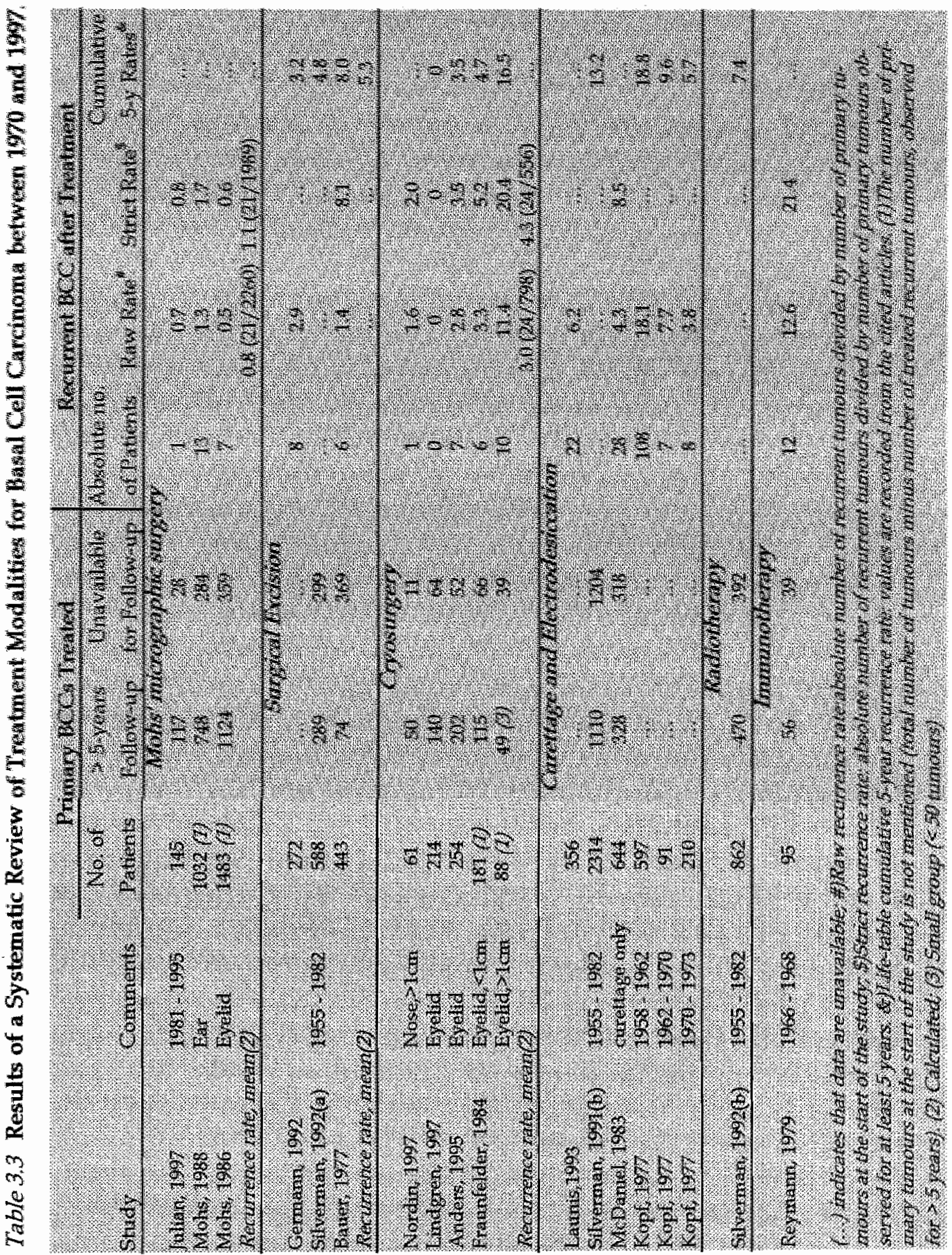


by Kopf $(18,1 \%)$ in the period 1958-1962. The cumulative 5-year recurrence rates for $\mathrm{CE}$ vary between $5,7 \%$ and $18,8 \%$.

\section{COMMENT}

Systematic reviews in clinical research are based on a few simple principles: systematically searching out, and, when possible, quantitatively combining the results of all studies that have addressed a similar research question (Egger, 1997). The literature on treatment modalities for primary (previously untreated) $\mathrm{BCC}$ and recurrence rates following therapy is massive. By systematically searching the available literature and trying to compare the recurrence rates for several therapies, it became clear that there is absolutely no uniform method of reporting the recurrence rates. From a statistical point of view, the recurrence rates are the results of calculating several types of survival curves. The most precise ones are the Kaplan-Meier survival curve (Parmar,1995) and the life-table survival curve according to Cutler and Ederer (1958), in which all available information about survival times is included. In this way "survival time" means the period in which the patient is free of recurrences. Unfortunately, only a few of the selected studies provided sufficient data to calculate the cumulative 5-year-recurrence rates according to the life-table method. Most of the (short-term) studies reported a recurrence rate based on total number of recurrences divided by the total number of initial tumours treated (raw recurrence rate). This method ignores the patients unavailable for follow-up and artificially lowers the recurrence rates reported. Thus, a recurrence rate of 5,0\% actually would be slightly higher if life-table analysis had been used. In contrast, the long-term studies reported a recurrence rate based on the total number of patients with recurrent $\mathrm{BCC}$ divided by the total number of tumours who were observed for at least 5 years (strict 5 year-recurrence rate). This method artificially raises recurrence rates, because it excludes patients with tumours who were observed for less than 5 years. The actual recurrence rate might be between these 2 values and best calculated by using the 5 -year life-table method. Therefore, an important initial conclusion of this review is the fact that the results of different treatment modalities for $\mathrm{BCC}$ cannot be compared based on the recurrence rates in the articles.

In theory, aggregation of data from multiple studies should enhance the precision and the accuracy of any pooled result. But combining data requires a leap of faith that the differences among studies are primarily due 
to chance. In fact, differences in treatment results are caused by other -often subtle-factors such as differences in populations, outcome measures, study design and study quality (Naylor, 1988). Thus, systematic reviews may generate misleading results by ignoring meaningful heterogenity among studies, entrenching the biases in individual studies, and introducing further biases through the process of finding studies and selecting results to be pooled (Egger, 1998).

Another goal of a systematic review is to possibly explain the variation in the results of equivalent therapies performed by different authors by looking at the factors that cause this heterogenity among the studies (Egger, 1997). For the studies of treatments of BCC the heterogeniety is large. From the literature, it is known that the risk for the recurrence of a treated $\mathrm{BCC}$ depends not only on the treatment modality, but also on the location (Kopf, 1979; Panje, 1979); on the size and histologic subtype of the tumour (Lang, 1986; Sexton, 1990) and to a lesser degree on patient-specific aspects such as age, immune status and sex (Bastiaens, 1998). Especially for BCCs of the micronodular, adenoid and morpheic subtypes and tumours localized in the $\mathrm{H}$-zone (danger zone) of the face, those larger than $2 \mathrm{~cm}$ are associated with a higher risk for recurrence. Those factors were initially left out of consideration, but after the included studies had been analyzed, it was obvious that they account for some of the high recurrence rates in several studies. Fraunfelder, for example, reported on the recurrence rate for BCCs on the eyelid, with those larger than $10 \mathrm{~mm}$ having nearly 4 times the recurrence rate of smaller BCCs in the same location (Fraunfelder, 1984). The 2 studies by Mohs were performed on BCCs located in a specific anatomic site, namely, on and around the external ear and on the eyelid (Mohs,1986 and 1988). Tumours located around the ear are known to have a higher risk for recurrence, so this might explain why the recurrence rate in this study (Mohs, 1988) was three times the recurrence rate in the first study (Mohs, 1986).

We assume that the choice for a specific treatment modality was also influenced by the features -location, size, and histologic type- of the specific tumour to be treated. The studies we analyzed did not randomize treatment as patients came in, because this was not practical or ethical. On the other hand, randomization is the only way to avoid tumour selection bias. This kind of selection bias can be seen especially in retrospective studies of treatments usually performed without previously drafted treatment protocols. For this reason, retrospectively analyzed studies were excluded in this review. 
Also important for the recurrence risk might be the period of treatment. The equipment needed for a particular treatment and the way in which some of the treatment modalities (especially $\mathrm{CS}$ and $\mathrm{CE}$ ) are performed today, might be more refined and precise compared with treatments performed earlier (Reymann, 1980). This could explain the high recurrence rates after $\mathrm{CE}$ both in the first group (treated between 1958 and 1962) of tumours reported on by Kopf et al. (1977) and in the study performed by Silvermann et al. (1991b), who described less favorable results in tumours treated in the earlier years (1955-1963). This explanation is only a hypothetical one, because other tumour specific factors could have attributed to these results. Nevertheless, to restrict the influence of the accuracy of the treatment in earlier decades, we analyzed only the studies published after the 1960s.

Ten years ago, Rowe et al. (1989) performed a meta-analysis on the recurrence rates for treatment modalities of primary BCCs. They also compared the recurrence rates after a follow-up shorter than 5 years with those of a follow-up longer than 5 years. Nearly two thirds of all recurrent tumours seemed to appear in the first 3 years after treatment, and $18 \%$ appeared between 5 and 10 years after treatment. According to this article, the reporting of recurrence rates for $\mathrm{BCC}$ should be standardized, using 5-year life-table analyses. We mostly agree with this statement; these 5 -year cumulative recurrence rates can best be compared in prospective studies, without a tumour selection bias. Nevertheless, studies reporting on treatment results after a follow-up of shorter than 5 years might give unrealistically low recurrence rates. For this reason, these studies were not included in our search.

Systematic review, in general, has made and continues to make major contributions to medical research, clinical decision making, and standards of research reportage; but it is no panacea (Naylor, 1997). When considering all the aspects mentioned earlier and shown in Table 3.3, MMS appears to be the treatment modality with the lowest recurrence rate, even for BCCs localized in anatomic sites at risk for recurrences. Several years ago, MMS was a time-consuming surgical method, but it can now be done efficiently. It was also thought to be an expensive treatment modality, but recently Cook and Zitelli (1998) demonstrated near equivalent cost in MMS versus standard SE with permanent section control and even lower cost in MMS versus SE with frozen section control. Treating all BCCs with MMS should certainly lead to overtreatment, especially in patients with smaller $(<2 \mathrm{~cm}) \mathrm{BCCs}$ of the nodular type located outside the H-zone of the face. These BCCs can be treated 
successfully with primary $\mathrm{SE}$.

According to the results of our analysis, the recurrence rates with CS and $\mathrm{CE}$ are higher than with surgical methods. No conclusions can be drawn from our results whether certain small tumours with less aggressive histologic subtypes localized on some areas of the body are treated better with CS or $\mathrm{CE}$. Further large, prospective comparative studies should be performed to analyze this. The recurrence rate for RT has been based on one solitary study (Silverman, 1992b), so it is difficult to draw a definite conclusion for this treatment modality. Because of the less favorable cosmetic results more than 5 years after therapy, this treatment modality should not be used as primary treatment in relatively young patients. Finally, the long-term cure after IM, so far based on the results of 1 study, is not promising (Reymann, 1979).

\section{CONCLUSIONS}

From this systematic review, it is not possible to propose general guidelines for the treatment of BCCs. Several characteristics of the tumour and the patient should be taken into account before deciding how to treat this specific tumour. If surgery is not contraindicated, SE remains the treatment modality of first choice for BCCs. For larger BCCs in the H-zone of the face, and those with more agressive growth patterns, MMS is recommended. Because the number of dermatologic surgeons, capable for performing MMS, is still low, special training in this surgical technique should be an important consideration for dermatologic surgeons. 


\section{Chapter Four}

\section{COSMETIC RESULTS OF CRYOSURGERY VERSUS SURGICAL EXCISION FOR}

PRIMARY UNCOMPLICATED BASAL CELL CARCINOMAS OF THE HEAD AND NECK

M.R.T.M. Thissen

F.H.M. Nieman

A.H.L.B. Ideler

P.J.M. Berretty

H.A.M. Neumann 


\begin{abstract}
Background: The incidence of multiple facial BCCs at younger age is increasing.

Objective: Cosmetic results after therapy become more important. Cosmetic results after cryosurgery and excision have never been compared prospectively in one study.

Methods: A randomized study was performed comparing cosmetic results one year

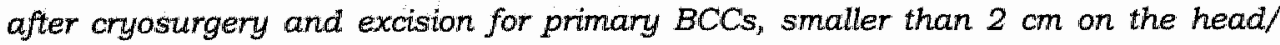
neck. Cosmetic results were assessed by independent observers (two dermatologists, plastic surgeon, nurse and beautician) and by the patients.
\end{abstract}

Results: $A$ total of 96 BCCs was treated either with surgical excision ( $n=48)$ or cryosurgery ( $n=48$ ). In general, clinical professionals evaluated the cosmetic results after surgery as significantly better than after cryosurgery, demonstrating even a relatively high grade of agreement within clinical professional pairs. The beautician had no preference for either therapy, while the patient had a significant preference for surgical excision. Lacalization and size of the tumour treated did not modify the general preference for surgical excision. The male dermatologist, however, had a tendency to evaluate the results in the cheek, periauricular, and neck area as inferior to the results in the rest of the face, regardless which therapy was given.

Conclusion: In general, cosmetic results after excision are better than after cryosurgery. Experience in dermato-surgery is necessary to offer the patient the best treatment, both in curative and in cosmetic aspect. 
Because more younger patients will develop multiple BCCs in the face in future, cure is no longer the only important aspect of treatment of $\mathrm{BCCs}$; the final cosmetic outcome of a certain therapy will become more important too. Surgical excision and cryosurgery are the two treatment modalities which are performed most frequently. However, the cosmetic results of these treatment modalities for primary BCC in general have never been compared prospectively in the same study. In this study, both the cosmesis and recurrences one year after cryosurgery and surgical excision of BCCs have been evaluated. However, determination of the recurrence rate was not the main goal of the study, because recurrences normally develop later and seldom in the first year after treatment (Rowe, 1989).

\section{PATIENTS AND METHODS}

\section{Study design and Patient selection}

The study was designed as a prospective, randomized, comparative study. Male and female patients between 18 and 80 years, with previously untreated, histopathologically proven BCCs of the nodular and superficial subtype were recruited from the outpatient department of dermatology of the Catharina Hospital in Eindhoven, the Netherlands between March 1996 and July 1998. Patients were selected referring to the following inclusion criteria: superficial or nodular BCCs, clinically smaller than $2 \mathrm{~cm}$ in diameter, localized anywhere in the head and neck area. Exclusion criteria were recurrent $\mathrm{BCCs}$, histologic subtypes different from the nodular or superficial subtype, tumours larger than $2 \mathrm{~cm}$ in diameter, patients with five or more $\mathrm{BCCs}$ and contra-indications to surgery or cryosurgery (e.g. cold intolerance). Patients with a life expectancy less than 1 year were also excluded.

Power analysis showed that at least 96 tumours were necessary to detect a $25 \%$ difference in the rate of good versus fair/bad cosmetic results (between $50 \%$ and $75 \%$ ).

\section{Treatment methods}

Patients were informed about the conditions of the study and after informed consent had been given, they were included at random for either cryosurgery or surgical excision. In case of multiple primary $\mathrm{BCCs}$, the lar- 
gest eligible tumour was assigned to the trial. The others were treated with the same technique.

Before treatment, the tumours were documented by taking photographs (SA-300N auto-focus reflex camera / zoomlens, Sigma, Japan; Kodak ISO 200-DX film) at two standardized distances (detail and overview), using standardized illumination. All treatments were performed at one single outpatient visit.

\section{Cryosurgery}

A thorough curettage, using a sharp Volkmann's curette, was performed under local anesthesia of lidocaine $1 \%$ with epinephrine. Initially, a large curette (no.3) was used to debulk the tumour mass. Finally, a small curette (no.1) was used to remove the remaining part of the $\mathrm{BCC}$ around the borders (Zacarian,1985). A swab dipped in $65 \%$ ferri-ferro-chloride solution was used to achieve haemostasis.

A liquid nitrogen spray unit (Frigitronics CS-76, Shelton Conn., USA) was used to freeze the tissue under an operating pressure of 8 psi. Freezing was carried out in two freezing periods, each lasting 20 seconds. The halothaw time between these cycles was 60 seconds (Torre,1990). All tumours were treated with the open cone spray technique, using a neoprene cone with a wall thickness of $2 \mathrm{~mm}$. The cone was modeled to a proper shape to enclose the tumour and a free margin of $5 \mathrm{~mm}$ around the tumour, and was pressed firmly against the underlying (bony) structure. If the tumour was too wide to be enclosed by the cone, freezing was performed in fractions.

After freezing, the treated area was covered by petrolatum dressings until the entire defect showed re-epithelialization.

\section{Conventional surgical excision}

Anesthesia was performed by local injection of lidocaine $1 \%$ without epinephrine. The tumour including a safety margin of $3 \mathrm{~mm}$ from the visible border was removed (Breuninger, 1984; Neumann,1996). Closure of the defect after undermining of the edges, was either primary by suturing in different layers, or by transposition or transplantation techniques according to the localization and size of the final defect. As usual, routine histologic examination of haematoxylin-eosin stained sections of the lateral and deep margins was performed in the first week to determine whether the removal of the tumour was complete or not, taking into account the shortcomings of this so 
called "breadloaf-technique" (Abide, 1984). In case of irradical treatment, one or more re-excisions were performed until all margins were free of tumour cells. Stitches were removed between 6 and 8 days after treatment, according to the localization.

\section{Follow-up}

Follow-up visits took place between day 6 and 8 (removal of the stitches, inspection of the cryo-defect), at day 30 and subsequently 3,6 and 9 months and finally 1 year after treatment. All patients were examined by the investigator for recurrent tumours.

At the last visit, photographs at two standardized distances were taken again from each tumour treated.

\section{Assessment cosmetic results}

The cosmetic results one year after treatment were independently assessed from the photographs taken at the last follow-up visit by five professionals who were not involved in the trial and who were blinded to the treatment. This panel consisted of a male dermatologist $(\mathrm{D}(\mathrm{m}))$, a female dermatologist/Mohs' surgeon $(\mathrm{D}(\mathrm{f})$ ), a plastic surgeon (PS), a dermatologic nurse (DN) and a beautician (B). Cosmesis was mainly evaluated by a three point scale (good, fair, bad).

In addition the patient was asked to rate the cosmetic result in points from 1 (very bad) to 10 (excellent), and also in terms of good, fair or bad.

\section{Statistics}

Data analysis was performed by crosstabulating the scale trichotomy with type of therapy (cryosurgery vs conventional surgical excision) and calculating the loglikelihood chi-square with 2 df for each (clinical) professional and the patient. In addition, a logistic regression analysis was performed on categorized evaluations (good vs fair/bad and bad vs good/fair) using type of therapy, location of treatment, size of the tumour in $\mathrm{mm}$ and the extent of sun-induced skin damage in the area operated upon as independent factors or variables. Forward selection and backward elimination techniques were used to determine the best fitting models for each evaluation. Agreement between evaluations of cosmesis among observers was measured by the kappa statistic. Odds ratios and partial odds ratios with $95 \%$ confidence intervals are given for each evaluator. Independent groups Student T-test was perfor- 
med for patient points measuring cosmetic results (Altman,1991). All data analysis was done using SPSS-pc, version 9.0.

\section{RESULTS}

In the period March 1996 until July 1998, a total number of 103 tumours was treated in this study. During the 12 months after treatment 3 patients did not appear for follow-up visits and 1 patient died (no relation with the tumour treated in this study). Another 3 patients developed a recurrent BCC (all were of the cryosurgery group) within the first year after treatment. The results presented here are the outcomes of the cosmetic results of 96 treated BCCs that had been followed for exactly one year.

\section{Patient characteristics}

The mean age of all patients treated was 68 years ( $\mathrm{s.d} .=13$ ); females were younger than males, although not statistically significant $(p=0.58)$. Five percent of the patients were younger than 50 years and the youngest patient was 35 years. In the cryosurgery group, the skin of 9 patients showed obvious signs of sun-damage, like changes in pigmentation and telangiectasia; in the surgery group this was seen in 17 patients (Table 4.1).

Table 4.1

\section{Tumour Characteristics of the Different Treatment Groups.}

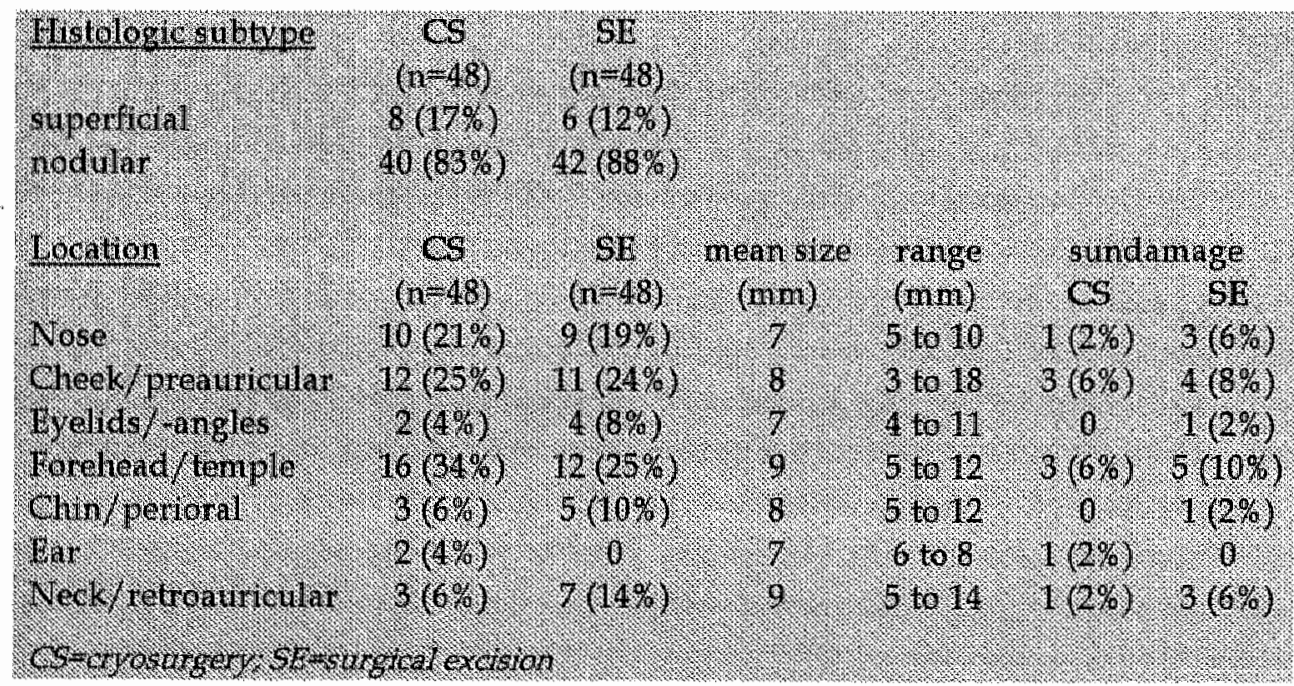




\section{Tumour characteristics}

Table 4.1 summarizes the characteristics of the BCCs treated in this study with respect to localization, histologic subtype and size for each of the two treatment groups.

\section{Cryosurgery}

In the cryosurgery group also 48 tumours were treated with liquid nitrogen, performing a standardized double freeze-thaw cycle. In nearly $90 \%$ (42 of the 48 tumours) of the cases patients complained of moderate to severe swelling of the treated area, followed by longlasting leakage of exsudate from the defect. Secondary wound infection, treated with systemic antibiotics (clarithromycin $500 \mathrm{mg} /$ day for 10 days) was seen in 3 cases $(6 \%$ ).

\section{Conventional surgical excision}

A total of 48 tumours was treated in the surgery group. Histopathologic examination of the resection borders showed complete tumour removal after the first excision in 43 tumours. For each of the 5 incompletely resected tumours ( 3 showed tumour remnants in the lateral borders and 2 at the bottom of the excision specimen), one re-excision of the scar and an extra margin of $3 \mathrm{~mm}$ performed within four weeks after the first excision, was enough to obtain complete removal of the tumour in all cases. Wound closure was performed by direct sutures in 36 cases $(75 \%)$ and transposition flaps in 11 cases $(23 \%)$. One $(2 \%)$ defect was closed by a full thickness graft. Two patients $(4 \%)$ developed secondary wound infections in the first and second week after surgery, for which systemic antibiotics (clarithromycin $500 \mathrm{mg} /$ day for 10 days) was started.

Serious complications like ectropion or damage to the lacrimal duct, were observed neither after cryosurgery, nor after surgical excision

\section{Failure rate}

Three tumours treated by cryosurgery showed recurrence within one year after treatment. All recurrent BCCs were treated subsequently by surgical excision with histopathologic examination of the resection margins. In the surgery group, no recurrences developed during the first year follow-up. 


\section{Chapter Four}

\section{Cosmetic results}

Table 4.2 shows the evaluation of cosmesis after cryosurgery and conventional surgical excision by professionals and the patient. In general, the clinical professionals evaluate the results after surgical excision as significantly better than the results after cryosurgery. The beautician has no preference for either therapy $\left(x^{2}=2.1\right.$ by $\left.2 d f_{;} p=0.35\right)$, while the patient does have a statistically significant preference for surgical excision $\left(x_{l}^{2}=6.4\right.$ by $2 d f ; p=0.011$. Clinical professionals are more critical on cosmetic results after therapy than the beautician and much more than the patient.

Table 4.2

Evaluation of Cosmetic Results aftex Cryosurgery ( $n=48$ ) and Surgical Exctsion ( $n=48$ ) by Professionals and Patients.

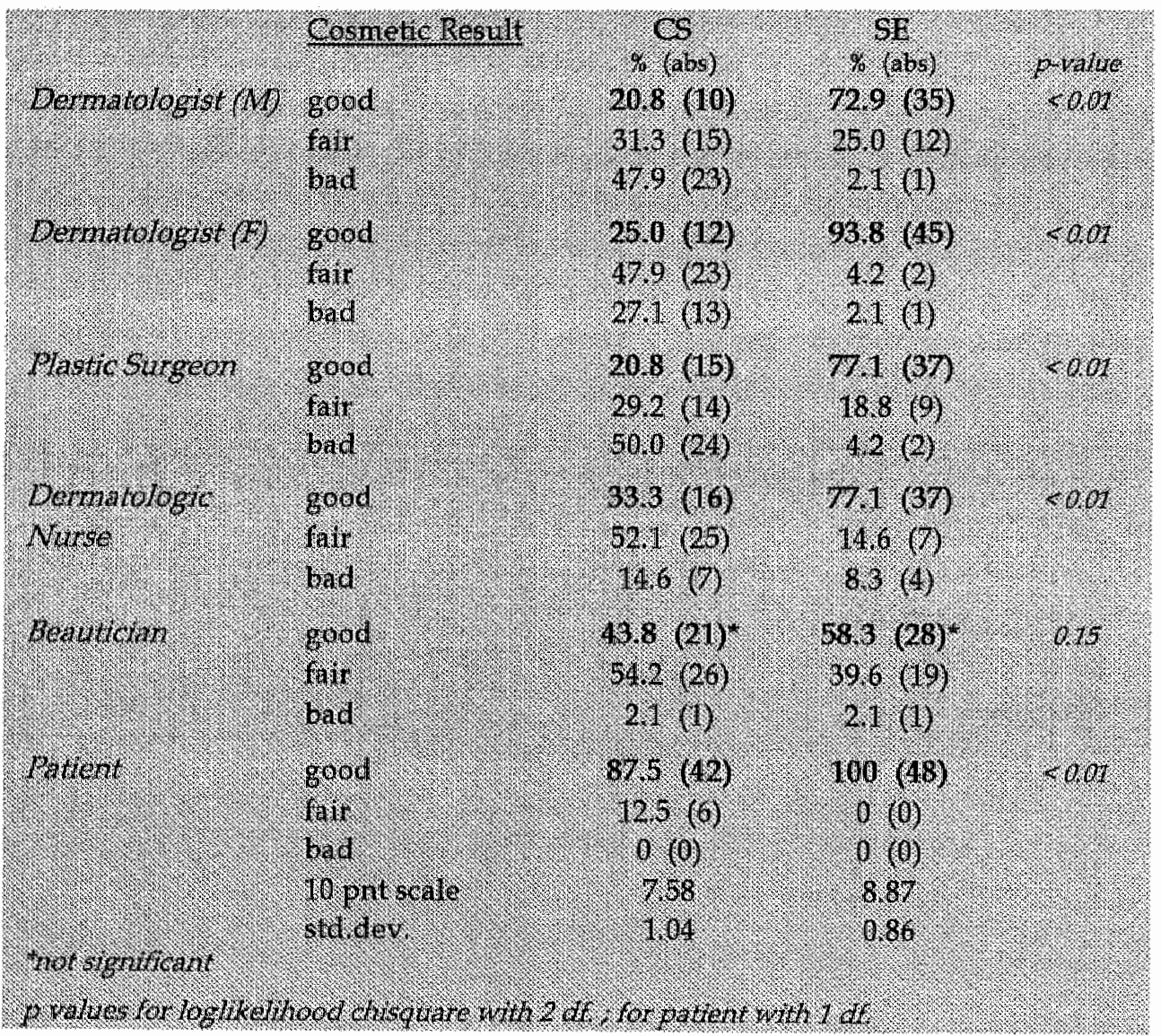


Table 4.3

\section{Agreement in Evaluation of Cosmetic Results between Professionals and Patients.}

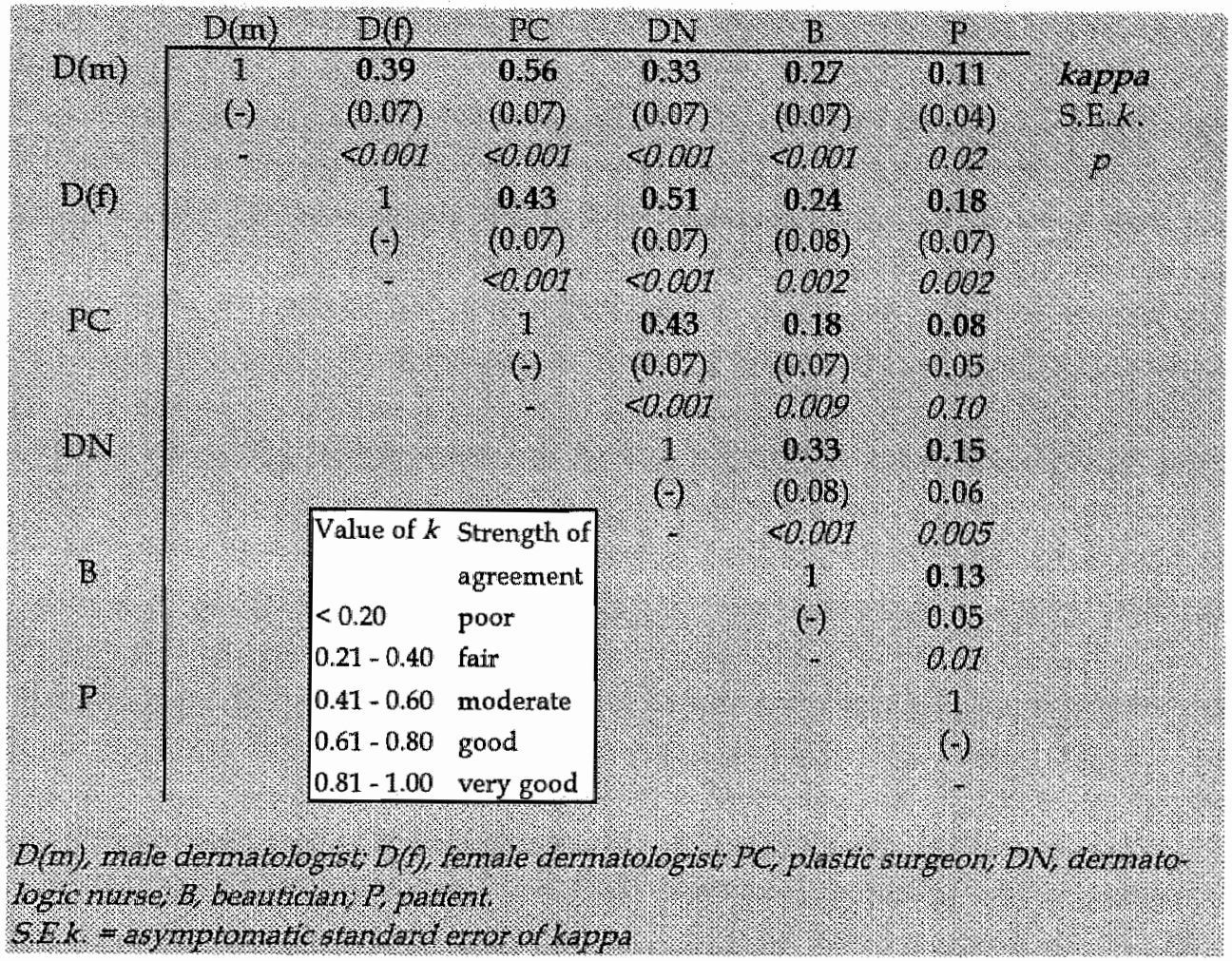

Table 4.3 shows the agreement in the evaluation of cosmesis, measured by kappa, between pairs of professionals and between professionals and the patient. Agreement within the clinical professional pairs ranges between 0.33 and 0.56 , while between clinical professionals and beautician it varies from 0.18 to 0.33 . Agreement is lowest between all professionals and the patient. $(0.08$ to 0.18$)$.

Next to therapy type, which was blinded for all professionals, we investigated, if and how much other (conditional) factors might modify the general preference for the results after surgical excision. Factors believed to have influence on the cosmetic result evaluation are: location of the surgical scar, length/size of the scar in $\mathrm{mm}$ and the extent of sun-induced damage in the area operated upon. The original codes in the evaluation $(1=$ good, $2=f a i r$, 
3 =bad) were recoded into "good" versus "fair-bad", and into "bad" versus "good-fair". Logistic regression analysis was run for each professional and for the patient separately, using forward selection and backward elimination techniques. For all clinical professionals and for the patient, the "mode of treatment" was again the only statistically significant factor, if the evaluation "good" versus "fair-bad" was used as a dependent variable. But in addition to this, the male dermatologist $(D(m))$ was also influenced in his judgement by the location of the surgical excision: both factors (therapy type and location) appear to strenghten in analysis, if the other is introduced into the evaluation of the cosmetic results as categorized above. No interactive effect could be found of both factors on the evaluation (change in $x^{2}=6.91$ by 4 df; $p=$ $0.141)$.

Table 4.4 shows the odds ratios for each professional separately, regarding "therapy" (and location), demonstrating a remarkable non-significance of "therapy" (odds ratio $<2$ ) in case of the evaluation of cosmesis by the beautician.

Table 4.4

Logistic Regression Results for Professionals: odds ratios showing the Preference of Cosmetic Results by Surgical Excision over Cryosurgery.

\begin{tabular}{|c|c|c|c|c|c|}
\hline & $18(\ln )$ & Der & les & DN & B. \\
\hline Mherapy" & & & & & \\
\hline Odartario & 18.2 & 50 & 12.5 & 6.7. & 1.8 \\
\hline privaring & 10.01 & 80.01 & +0.01 & +106 & 0.15 \\
\hline $96 \% 8$ & & 11.100 & $(17=33$. & $27-167$ & $9.8-4$ \\
\hline $\mathrm{asal}^{\prime}$ & & & & & \\
\hline Odds raifo & 0.23 & & & & \\
\hline prvarine & 007 & & & & \\
\hline $95 \%$ & $0.08 \cdot 071$ & & & & \\
\hline (Tharary s: & 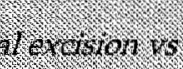 & (osvitger) & $\operatorname{ligh}(>1)$ & Tato st & oprete \\
\hline ence $60 \mathrm{r}$ ores & vis -110 & Hortar & or a r preres & 1or ces & \\
\hline 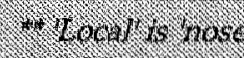 & endron & drest & edr. pro & thon & (ne) \\
\hline 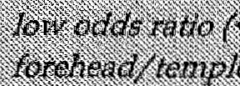 & rirt & & & & \\
\hline
\end{tabular}




\section{COMMENT}

This study demonstrates that in general, cosmetic results after surgical excision for primary BCCs of the superficial and nodular subtype localized in the head/neck region are significantly better than after cryosurgery. Although several studies reported evaluations of cosmetic results after different treatment modalities for $\mathrm{BCC}$ as an additional study object, overall cosmetic results after surgical excision and cryosurgery had not been compared prospectively in one study before for this indication. Previously, this was done by Beard, but only for both primary and recurrent BCCs of the eyelids (Beard,1975). All treatments in our study were performed by experienced medical professionals, practising in an average outpatient dermatologic clinic. The cosmetic results were assessed by an independent panel. The members of this panel were not involved in the treatment procedures, giving the opportunity to obtain unbiased results.

The cosmetic results in this study were assessed in general for both groups of patients treated in this study. Patient dependent factors like the skintype and age have not been included in this analysis. The influence of the amount of sun-exposure resulting in a certain grade of sun damage, as mentioned in Table 4.1, was not taken into account. The reason for this was the fact that it was not the primary goal of this study to evaluate the impact of different factors on the final cosmetic effect. We only had the intention to let the cosmetic results after surgery and cryosurgery be evaluated in an average population, in an average dermatologic setting. After treatment of the tumour and the surrounding skin by different physical and invasive treatment modalities the time needed for recovery of the treated area depends on the specific therapy. Both surgical and cryosurgical scars will reach their final appearance during the first year after treatment. The definitive cosmetic results after radiotherapy on the other hand can only be achieved after several years (Avril,1997). We have decided to evaluate the cosmetic results one year after treatment, because we thought this interval is the maximum time period that would be acceptable for the patient to see how the final cosmetic outcome of a typical treatment will be.

By using a 3-point score scale, we kept the level of variations in the evaluation of the cosmesis to a minimum. Despite the relatively high levels of agreement among the clinical professionals, the evaluation of the results after surgical excision by the male dermatologist was somehow different from the others, because he was the only observer whose judgement was influ- 
enced by the location of the tumour. The ratio behind his evaluation can be interpreted as follows. Because cryosurgery is somewhat less (but not statistically significant) applied to cheek/preauricular or neck/retroauricular locations than surgical excision, and cryosurgery results are more likely to be evaluated as "fair-bad" by the male dermatologist than surgical excision results, his final evaluation of cheek/preauricular or neck/retroauricular operations -compared to those on other locations- will be clearly and significantly more negative than the male dermatologist claims at first glance. Otherwise it can be stated that, because cheek/preauricular or neck/retroauricular locations are somewhat more (but not statistically significant) operated upon by surgical excision than by cryosurgery, and cheek/preauricular or neck/retroauricular operations are usually (but just not significantly) judged as "fair-bad" by the male dermatologist, his initial clearly positive evaluation of the results after surgical excision must be corrected to an even more clearly stated preference of this technique over cryosurgery.

Compared with the scores of the panel, the evaluation of the cosmetic results by the patients was quite different. According to their evaluations, surgical excision (with $100 \%$ good results) also lead to better cosmetic results than cryosurgery, although the difference was very small. However, they judged only $12 \%$ of the results after cryosurgery as "fair" and even $0 \%$ as "bad" as they were asked for the cosmetic results by the person who had treated them. An explanation for this might be the fear of the patient for disturbance of the patient-doctor relationship by judging the results as less favorable. However, this seems less plausible, because also their anonimous judgement of the scale of satisfaction after cryosurgery was better than the judgement of the panel. Another explanation might be the fact that the final cosmetic outcome does not impress the patient as much as the fact that he has been cured of his cancer, so the good score more likely expresses the latter aspect.

The rate of "good" cosmetic results after surgery, as demonstrated in this study, is consistent with previously published data concerning this subject (Robins, 1985; Marchac, 1988; Hohmann, 1992, Silverman,1992; Bonvallot, 1993), ranging from $77 \%$ to $93 \%$. For the rates of "good" cosmetic results after cryosurgery on the other hand, the situation is quite different. Good to excellent results have been reported for tumours on the eyelids (Biro, 1982; Tuppurainen,1995; Lindgren,1997), or even generally for skin cancer localized anywhere on the body (Kuflik, 1991). Less favorable results have been reported for 
lesions on the nose (Biro,1982; Nordin "1997). The reason for this discrepancy could be the fact that in our study the results are directly compared to each other, which was obviously in favor for surgical excision.

\section{CONCLUSION}

Incidence rates for $\mathrm{BCC}$ are still rising all over the world. Besides that, more and more patients are developing multiple tumours at a younger age, most of which are localized in the face. Cure, however, remains the main goal of cancer therapy, but for the treatment of BCCs it will become more important for dermatologic professionals to pay attention to the final cosmetic appearance after therapy. Surgical excision should be the therapy of first choice for treatment of primary BCCs. Despite the time-saving and low-cost aspect of cryosurgery, this therapy should be preserved for patients in whom contraindications to surgery exist, or for small superficial and nodular BCCs localized on the eyelid/inner eye angle and helix.

For these reasons, "the dermatologist of the future" should be broadly experienced in the performance of surgical excisions to keep up high standards in the state of the art and provide each patient with the best treatment for his $\mathrm{BCC}$, both in terms of cure and from a cosmetic point of view.

\section{Acknowledgement}

I would like to thank the following professionals for their participation in this study: Dr. RC Beljaards, dermatologist; Drs. JU Ostertag, dermatologist/Mohs' surgeon; Drs. A Damen, plastic surgeon; Mrs. I Habets, dermatologic nurse; Mrs. L Hamstra, beautician; Mr. G v Dael, medical photographer. 


\title{
Chapter Five
}

\section{TREATMENT OF BASAL CELL CARCINOMA}

BY

\section{DERMATOLOGISTS IN THE NETHERLANDS}

\author{
M.R.T.M. Thissen \\ H.A.M. Neumann \\ P.J.M. Berretty \\ A.H.L.B. Ideler
}

Ned Tijdschr Voor Geneeskd 1998;142:1563-7.

WCS News 1999;15:35-8. 


\begin{abstract}
Objective: To determine the policy in the treatment of basal cell carcinoma of dermatologists practising in the Netherlands.

Design: Written inquiry.

Setting: Catharina Hospital, Eindhoven, the Netherlands.

Method: All 293 dermatologists practising in the Netherlands recieved a questionnaire in May 1996 containing 15 questions about diagnosis and treatment of basal cell carcinoma.
\end{abstract}

Results: Eighteen forms dropped out because of termination of the practice or joint completion in group practices. The response was $76 \%(208 / 275)$. The diagnosis was made usually on the basis of histological examination $171 \%$ of the respondents; $84 \%$ in recurrent tumours). Excision was the preferential treatment for all subtypes of basal cell carcinoma; second choices were cryosurgery or curettage/ electradesiccation. Roentgen contact therapy has been practically abandoned. New methods such as photodynamic therapy and topical immunotherapy are being used only sponadically on an experimental basis. Most dermatologists regarded recurrent tumours as a bigger problem than primary tumours. They attempt to reduce the percentage of recurrences by giving advice about risk factors (sunlight).

Conclusion. Too little use is being made of diagnostic biopsy to enable an optimal choice of therapy of basal cell carcinomas, especially in cases of recurrent tumours. 
Among the dermatologists in the Netherlands, an inquiry has been performed to obtain an overview of the way in which they diagnose and treat BCCs. Another aim of this inquiry was to become informed about the number of new BCCs diagnosed yearly in our country.

\section{METHODS}

A questionnaire (next page) was sent to all dermatologists, who are practising in the Netherlands, and they were asked to fill in and return the form anonymously as soon as possible. Their adresses were obtained from the Dutch Society for Dermatology and Venereology. The questionnaire consisted of fifteen questions with regard to diagnostic procedures and therapy for $\mathrm{BCC}$, and advice to prevent further development of $\mathrm{BCCs}$. The questions could be answered either with "yes" or "no" (followed by specification if asked for) or by giving percentages. For the questions about the treatment of $\mathrm{BCC}$, a distinction was made between the different histologic subtypes and primary or recurrent $\mathrm{BCCs}$.

\section{RESULTS}

In May 1996, a total number of 293 questionnaires have been sent to the dermatologists. Of these, 18 forms dropped out, 2 because of termination of practice, and another 16 because one single form represented the results of the total department of dermatology. After three months, 208 copies had been returned, which implies a response of $76 \%$.

From the questionnaire it became clear that an estimated number of 90 to $100 \mathrm{BCCs}$ is diagnosed in one year by a dermatologist in the Netherlands. The annual incidence in the Netherlands, calculated from these results, approximately amounts to a total number of 29.000 BCCs. Most tumours are primary, solitary BCCs. Recurrent $\mathrm{BCC}$ is diagnosed in $10 \%$ of the cases, and in $5 \%$ of the patients multiple BCCs are diagnosed. Seventynine percent of the dermatologists considers a recurrent tumour more problematic than a primary tumour (larger, deeper localized). To confirm the diagnosis of $\mathrm{BCC}, 71 \%$ of the dermatologists always takes a biopsy for histopathologic examination from each solitary eruption suspected of a primary BCC. In cases suspected of recurrent BCC, a biopsy is taken by $84 \%$ of the 


\section{Questionnaire \\ "Treatment of Basal Cell Carcinomas by Dermatologists in the Netherlands"}

\section{Diagnostic procedures}

* Do you always take a biopsy for histopathologic examination from each lesion in case of a solitary eruption suspected of primary $\mathrm{BCC}_{\text {, }}$ multiple eruptions suspected of primary BCC, and eruptions suspected of recurrent BCC? (if "no", specify).

* Indicate the number of patients, and the amount of primary and recurrent BCCs you have diagnosed last year.

\section{Therapeutic procedures}

* Indicate your preferential treatment ${ }^{*}$ and your second- and third-line treatment", separately for both primary and recurrent $\mathrm{BCC}$ of the superficial, the nodular and the morpheic/micronodular histologic subtype surgical excision, Mohs' micrographic surgery, cryosurgery, curettage/electrodesiccation, radiotherapy, immunotherapy, chemotherapy, photodynamic therapy)

* Do you prefer any other treatment modality, not mentioned in the previous question, in specific situations? (if "yes", illustrate)

* Is your preference for a therapy influenced by one or more of the following factors: histologic subtype, location or clinical demarcation/size of the BCC; multiple BCCs in one patient; general health or age of the patient? (if "yes", specify)

* Do you prefer to combine two or more treatment modalities in a specific situation? (if "yes", illustrate)

* Do you prescribe retinoids or 5-fluorouracil after treatment of a BCC to diminish the risk for recurrences?

* Indicate the percentages of both primary and recurrent BCCs of the superficial, nodular and morpheic/micronodular subtype you treat by yourself.

* Do you refer patients for treatment of BCC to other specialists? Specify the indications for referral (e.g. size/localization/subtype of the BCC, age/general health) and the department referred to (e.g. Mohs' surgeon, radiotherapy, plastic surgeon).

* What will be your policy in cases where histologic examination after surgical excision demonstrates non-radical removal of the tumour : re-excision, adjuvant therapy (e.g radiotherapy) or "wait and see" ? (see also next question)

Which of the following factors will influence your decision to omit further treatment in case of non-radical removal: histologic subtype/localization of the tumour, primary or recurrent $B C C$, re-examination of the resection margins by yourself/localization of the tumour rests, general health/age of the patient? (if "yes", please specify)

* Do you consider a recurrent BCC as a bigger problem than a primary BCC? (if "yes", specify)

\section{Follow-up and Prevention}

* For how many years do you follow a patient with $1 \mathrm{BCC}$ no follow-up, 1 year, 3 years, 5 years, 7 years, life-time?

* For how many years do you follow a patient with multiple BCCs: no follow-up, 1 year, 3 years, 5 years, 7 years, life-time?

Do you provide your pattient with information/advice with regard to risk factors (e.g. avoiding excessive sun exposure, use of sunscreens) for development of BCCs: always, only younger patients ( $<50$ years), seldom, never? 
Table 5.1

\section{Preferential Treatment $(\%)$ and Other Therapies $(\%)$ used as the First- Line Treatment for Primary and Recurrent Basal Cell Carcinomas by Dermatologists in the Netherlands.}

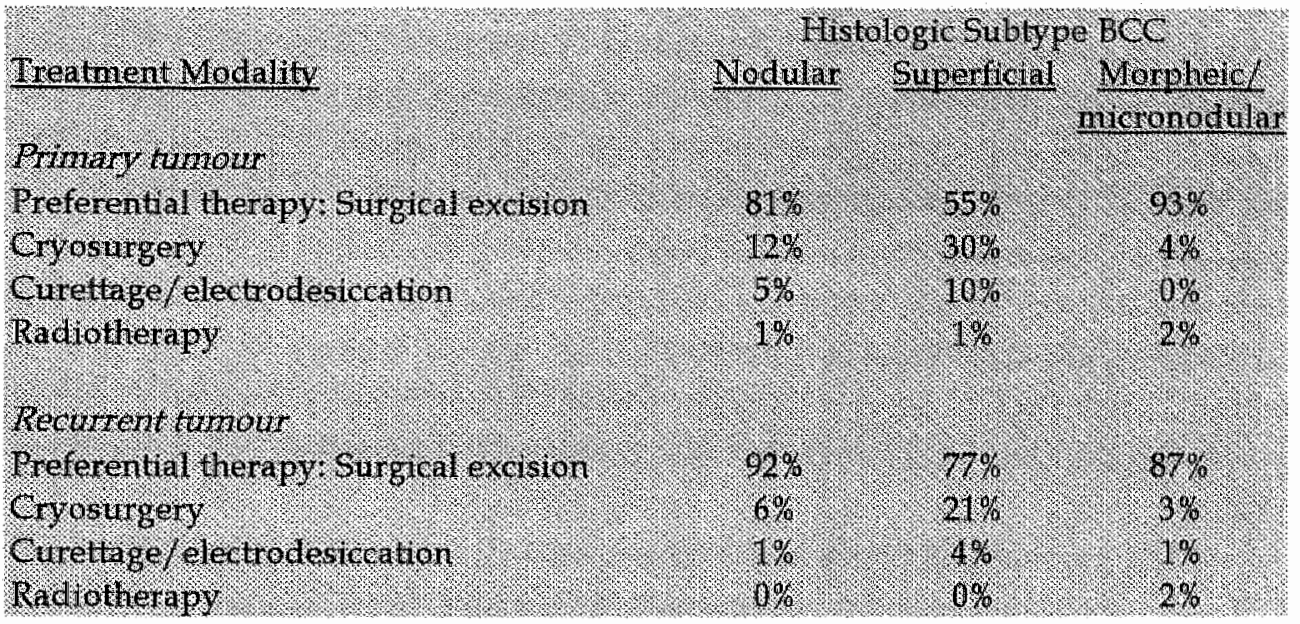

dermatologists. In patients presenting with more than one lesion suspected of $\mathrm{BCC}, 57 \%$ takes biopsies from all these lesions.

For nearly every dermatologist the localization (96\%) and the clinical demarcation of the tumour $(91 \%)$, the age and the general health of the patient $(91 \%)$ are important factors that influence the decision for a specific therapy. Eighty-one percent of the BCCs is treated by dermatologists themselves. The remaining number is referred for radiotherapy (elderly persons, contra-indications to surgical intervention) or plastic surgery (larger BCCs, localized around the eyes and on the nose).

Surgical excision is the preferential treatment for all histologic subtypes of both primary and recurrent BCCs (nodular $81 \%$, superficial $55 \%$, and morpheic/micronodular $93 \%$; Table 5.1). Re-excision is performed by $73 \%$ of the dermatologists, if histopathologic examination shows tumour cells in the resection margins. In these cases, an expectative policy is pursued by $23 \%$, especially if the tumour remnants are localized in the lateral margins. Sporadically (4\%) adjuvant radiotherapy is given. In cases where surgical excision was incomplete (not radical), a higher age of the patient (70 $\%$ ) and a localization of the BCC on the cheek, the forehead or the chin, are 
important factors for the decision to omit additional therapy. Furthermore, 9 $\%$ of the dermatologists never performs a surgical excision for superficially growing BCCs. Mohs' micrographic surgery was mentioned as the preferential treatment for larger, morpheic/micronodular BCCs by a few of the dermatologists. Cryosurgery is the first alternative therapy, especially for the superficial subtype. Curettage/electrodesiccation and radiotherapy are mentioned as the second alternatives for all subtypes. An overview of the percentages in which cryosurgery, curettage/electrodesiccation and radiotherapy are used as first-wine treatment in primary and recurrent BCCs is shown in Table 5.1. Immunotherapy and photodynamic therapy are only used in experimental sessions. In cases of multiple BCCs in one patient, $57 \%$ of the dermatologists uses topical chemotherapy (5-fluorouracil) or systemic therapy with methotrexate or retinoids as. Topical 5 -fluorouracil is used by $32 \%$ of the dermatologists as adjuvant therapy to diminish the risk for recurrences.

Tumour follow-up to check for recurrent or new BCCs is performed by $24 \%$ for a minimal period of three years, and by $68 \%$ for five years. Patients with multiple BCCs are followed for a period of five to seven years by $58 \%$, and life-time by $39 \%$.

Advice with regard to avoiding the riskfactors for $\mathrm{BCC}$, like (excessive) exposure to sunlight, is given by $80 \%$ of the dermatologists. Fourteen percent gives this information only to patients younger than 50 years of age.

\section{COMMENT}

The results of the inquiry give an overall view of the way in which diagnostic procedures and treatment of BCCs is performed by the dermatologists in the Netherlands. Because of the high response rate, the results of this inquiry can be assumed to be representative for the average dermatological practice. It seems that the recommendations of the Dutch Society for Dermatology and Venereology concerning the treatment of BCCs, as written in the "Technical guidelines of the Advisory Board for Oncology and Surgical Dermatology", are followed for the greater part.

Initially, a representative, full-thickness biopsy from every eruption suspected for $\mathrm{BCC}$ should be taken for histopathologic examination (Neumann, 1996a). The biopsy should be incised deeper or repeated in cases where the clinical diagnosis cannot be confirmed histopathologically. Surgical excision in toto without preceding biopsy should be limited to patients who pre- 
sent with multiple lesions suspected for BCC. Unfortunately, in the Netherlands $\mathrm{BCC}$ is diagnosed by $29 \%$ of the dermatologists on clinical examination only. Without taking a previous biopsy, in certain patients the most appropriate therapy might not be used, because only on the basis of the biopsy information can be obtained about the growth pattern of the tumour In small BCCs where the biopsy site heals without leaving clinical evidence of residual tumour, further therapy is recommended however, because there is a serious risk for recurrence of the tumour in future (Holmkvist, 1999).

Several factors like location, size and histologic subtype of the tumour, age and general health of the patient, and the experience of the dermatologists who is treating the patient, play an important role in the final decision which therapy should be the best for a typical tumour. Today, with the actual treatment modalities, $90 \%$ of the BCCs can be cured primarily. This number corresponds with the percentage of recurrent $\mathrm{BCCs}$ diagnosed by the dermatologist in the Netherlands.

The inquiry shows that surgical excision is the preferential treatment for all subtypes of $\mathrm{BCC}$. The resection margins can be examined afterwards for completeness of the tumour removal (Wolf, 1987). On the other hand, parts of the tumour (especially BCCs growing with fine off-springs or with perineural extension) might be missed, if routine histopathologic examination of the excised specimen is performed. For this reason, a fully three-dimensional picture of the resection margins, as obtained during Mohs' micrographic surgery, should be available to determine whether the tumour has completely been removed (Neumann,1996b). For practice, it will not be necessary to examine each $\mathrm{BCC}$ histopathologically in a three dimensional way. Accurate histopathologic examination, however, is required for morpheic, micronodular, and adenoid growing BCCs. Mohs' micrographic surgery is an excellent treatment for $\mathrm{BCCs}$ on difficult locations and tumours with clinical invisible borders, combining optimal cure with removing as little healthy tissue as possible (Tromovitch, 1978; Krekels, 1996a). Recurrence rates after Mohs' micrographic surgery are 1-2 \% for primary tumours (Mohs, 1978; Robins, 1984) and 7 $\%$ for recurrent tumours (Rowe,1989). In the Netherlands, at this moment Mohs' micrographic surgery is performed in a few centres only. This might be the explanation for the fact that Mohs' micrographic surgery is mentioned as preferential treatment only by a few of the dermatologists. Extension of the number of Mohs' centres is desired with regard to the increasing incidence rate of (clinically invisible and more agressive) BCCs. Today, more derma- 
tologists are educated in this technique (Krekels, 1996b).

Although only surgical techniques followed by adequate histologic examination can lead to the assumption that the tumour has been completely removed, the physical therapies like cryosurgery and curettage/electrodesiccation, lacking this control, are used frequently by the dermatologists in the Netherlands. These treatment modalities should be reserved for the small (superficially growing) $\mathrm{BCCs}$ outside the $\mathrm{H}$-zone, in patients with contraindications to surgery. Cryosurgery is even a preferential treatment for BCCs on the eye-lid and the helix (Gunnarson, 1990).

For the treatment of large $\mathrm{BCCs}$, patients are referred frequently for plastic surgery or radiotherapy. During the last years, radiotherapy has become less popular as a treatment for BCC, because of the inferior long term cosmetic results and the difficulty of surgery for recurrent tumours. Today, radiotherapy is mainly used for treatment of $\mathrm{BCC}$ in elderly with contraindications to other therapies. The effectiveness and safety of the new treatment modalities, topical interferon and photodynamic therapy, should be investigated first in large, prospective clinical trials with long term follow-up.

\section{CONCLUSIONS}

This inquiry revealed that the annual number of BCCs diagnosed in the Netherlands seems to be high and still increasing, in conformity with the estimate of the Dutch Health Committee. A large percentage of the BCCs is treated by dermatologists themselves. The importance of adequate diagnostic procedures (reliable histopathologic examination) and correct treatment of the BCC (radical treatment) must be emphasized. The percentage of diagnostic biopsies is low, certainly for recurrent BCCs. Inadequate treatment might result in the development of larger recurrent $\mathrm{BCCs}$, for which more serious intervention will be necessary.

Finally, adequate tumour follow-up is important for each patient with one or more BCCs; recurrent and new BCCs can be detected in an early phase of development, which makes them easier to treat. Guidelines for this follow-up have also been proposed by the Dutch Society for Dermatology and Venereology (NVDV-Guidelines Technical Commission).

\section{Acknowledgement}

This study could have not been performed without the help of the dermatologists in the Netherlands. I would like to thank all of them for the efforts they made. 


\section{Chapter Six}

\section{EFFECTIVE PHOTODYNAMIC THERAPY WITH 5-AMINOLEVULINIC ACID FOR NODULAR BASAL CELL CARCINOMAS USING A PRECEDING DEBULKING TECHNIQUE}

M.R.T.M. Thissen

C.A. Schroeter

H.A.M. Neumann

Br J Dermatol 2000;142:338-9. 


\section{Abstract}

Background: The incidence of $B C C$ is still increasing and there is a demand for easy, effective, and selective, non-invasive treatment.

Objective: To evaluate the effectiveness and safety of topical photodynamic therapy (PDT) with 5-aminolevulinic acid (5-ALA) as a photosensitizer and the Versalight as the lightsource, in combination with a preceding debulking technique, for the treatment of noduiar $B C C$.

Methods: Twenty-three patients with 24 nodular BCCs were treated once with 5-ALA - PDT (acclusive application of $20 \%$ 5-ALA emulsion for 6 hours followed by irradiation with $630 \mathrm{~nm}, 100 \mathrm{~mW} \mathrm{~cm}, 120 \mathrm{~J} \mathrm{~cm}^{-2}$ ) three weeks after a preceding debulking of the BCC. Three months after PDT all lesions were surgically excised and histopathologically evaluated for tumour remnants.

Results. Twenty-two (92\%) of the 24 nodular BCCs showed complete response on clinical and histopathological examination. Serious side effects were not observed. Cosmetic results were excellent in all treated patients.

Conclusion: PDT for partially abraded nodular BCC with topically applied 5-ALA and the Versalight ${ }^{R}$ as lightsource might be an effective and safe therapy, with excellent cosmetic resuits and no serious side effects, in cases where non-surgical treatment of $B C C$ is indicated. 
Photodynamic therapy (PDT) using 5-aminolevulinic-acid (ALA) induced protoporphyrin IX (PpIX) as a photosensitizer is used as "a treatment under investigation" for a variety of malignant and non-malignant disorders (Malik, 1987; Loh, 1992; Jones, 1992; Szeimies, 1996; Fritsch, 1998). Since Kennedy first described the treatment of superficial skin carcinomas using ALA-PDT, other centres have reported the effectiveness of this type of treatment (Kennedy, 1990; Wolf, 1990; Wilson, 1992; Cairnduff, 1994; Svanberg, 1994; Wennberg, 1996). A complete initial clearance of more than $90 \%$ has been reported for treatment of superficial lesions like superficially growing basal cell carcinoma (BCC), Bowen's disease and solar keratosis with topically applied ALA-PDT. However, for thicker lesions such as nodular BCCs a much lower complete response of $50 \%$ could been obtained with topical ALA-PDT (CalzavaraPinton, 1995). An explanation for this lower efficacy might be insufficient penetration into the deeper layers of the tumour of either topically applied ALA or the light.

The purpose of this study was to investigate the influence of a preceding partial debulking of the tumour mass, with the intention to obtain a deeper penetration of both the sensitizer and the light, on the final effectiveness of PDT for nodular BCCs, evaluated by surgical excision and histopathologic examination of the treated tumours three months after PDT. The side effects and the cosmetic results of PDT were also documented.

\section{PATIENTS AND METHODS}

\section{Patient Selection}

Male and female patients, between 18 and 80 years of age, with prima$\mathrm{ry}$, histopathologically proven $\mathrm{BCCs}$ of the nodular growing type, were recruited for entry into a prospective open pilot study from the outpatient department of dermatology, the Catharina Hospital in Eindhoven, the Netherlands. Patients were selected according to the following inclusion criteria: 1$3 \mathrm{BCCs}$, clinically smaller than $2 \mathrm{~cm}$ in diameter, localized anywhere except on the eyelid.

\section{Materials}

A freshly prepared cream ( $w / o$ ) (Neribas, Schering, the Netherlands), 
containing 20\% 5-ALA (hydrochloride, Merck, the Netherlands) as the active ingredient, was used. For illumination, the VersaLight ${ }^{R}$ (ESC Medical Systems, Yokneam, Israel), an intense continuous wave, polychromatic, incoherent light delivery system with adjustable power, was used. The specific wavelength could be selected by using cut-off filters.

\section{Treatment protocol}

Three weeks before PDT (at the same time the punch biopsy for histopathologic confirmation of the diagnosis was taken, using local anesthetics), a partial debulking of the tumour mass was performed, using a sharp disposable curette. For the final treatment, the 5-ALA cream was applied in the gap of a large transformable, synthetic ring that was stuck to the skin, surrounding a margin of at least $0.5 \mathrm{~cm}$ of the normal skin around the tumour. In this way, the light sensitive area could be kept as small as necessary. Subsequently, the tumours were kept under light reflecting, occlusive dressings. After 6 hours, the illumination was performed, using the red light emissionspectrum (585 - $720 \mathrm{~nm}$ ) including the $630-636 \mathrm{~nm}$ optimum for 5ALA (Svanberg, 1994). A total light dose of $120 \mathrm{~J} \mathrm{~cm}^{-2}$ (spotsize $3 \mathrm{~cm}$, light intensity of $100 \mathrm{~mW} \mathrm{~cm} \mathrm{~cm}^{-2}$ ) was delivered to each tumour in a continuous way. Patients were reviewed on day 1, 7, 28 and 84 after treatment. At each visit, standardized photographs were taken for documentation of the healing process and the cosmesis.

Three months after PDT, the treated skin area was totally removed by surgical excision followed by histopathologic evaluation for tumour remnants, using the "breadloaf"-technique (examination of multiple vertical cuttings). Before the excision was performed, the cosmetic results of PDT were assessed by using a subjective scoring system by both the investigator and the patients: $1=$ bad (atrophy, teleangiectasia, and severe hypopigmentation); 2 moderate (obvious hypopigmentation); $3=$ good (slight hypopigmentation); and $4=$ excellent (no skin changes).

As control groups served three BCCs treated by debulking and application of 5-ALA only (no illumination), three BCCs treated by debulking and illumination only (no 5-ALA), three BCCs treated by PDT without preceding debulking, and another three BCCs treated only by debulking (no PDT).

\section{RESULTS}

Twenty-three patients with 24 primary, small $(<1 \mathrm{~cm})$ histologically pro- 
ven nodular BCCs localized on the face, neck and trunk were included in the study. During the illumination, two patients mentioned a moderate burning sensation. Erythema and edema were seen on and $1 \mathrm{~cm}$ around the tumours immediately after the illumination, resulting in hyperpigmentation and formation of crusts during the first week after PDT in all cases, followed by uncomplicated healing. No other serious side effects have been reported. On clinical examination three months after PDT, 2 of the treated BCCs were suspected of tumour remnants. Routine histopathologic examination of all treated BCCs showed tumour rests in exactly the same $2 \mathrm{BCCs}$. The cosmetic results after PDT were assessed as good in 1 and excellent in 21 of the 22 cured BCCs. Scar formation was not observed (Figure 6.1).

All $\mathrm{BCCs}$ in the control groups remained clinically visible after three months, and showed tumour cells on histopathological examination.

\section{COMMENT}

There are several therapies for the treatment of BCC (Motley 1995). In general, surgical excision is the therapy of first choice, but in cases where surgical intervention is contraindicated, a non-invasive treatment is preferred. Radiotherapy and cryosurgery, for example, are non-surgical (i.e. non-sharp) treatment modalities, leading to destruction of both tumour cells and surrounding normal tissue, resulting in cosmetically disturbing discolorations and scars. For this reason, there is a demand for a more selective, non-invasive treatment like PDT (Fritsch, 1997). Until now, BCCs have already been treated with PDT only in small, not standardized clinical studies with short follow-up. Shortterm complete clinical response rates, reported in these studies, vary between $90 \%$ and $100 \%$ for BCCs of the superficial subtype, and $10-64 \%$ for BCCs of the nodular subtype (Wolf, 1993; Svanberg, 1994). PDT is still "a treatment under investigation"; there is a lack of large, prospective clinical studies, based on standardized treatment protocols and adequate follow-up. So far, the clinical response rates of PDT can not be compared with the cure rates of other treatment modalities.

In this study, the effectiveness of PDT for nodular BCC was verified three months after PDT, by surgical excision and histopathologic examination (breadloaf-technique) of the treated tumours. This was done to overcome the long waiting time for the standard 5-year disease free survival at this moment. The exact site for excision could be recognized with the help of the detailed photographs taken before PDT. Even if this breadloaf-technique is 

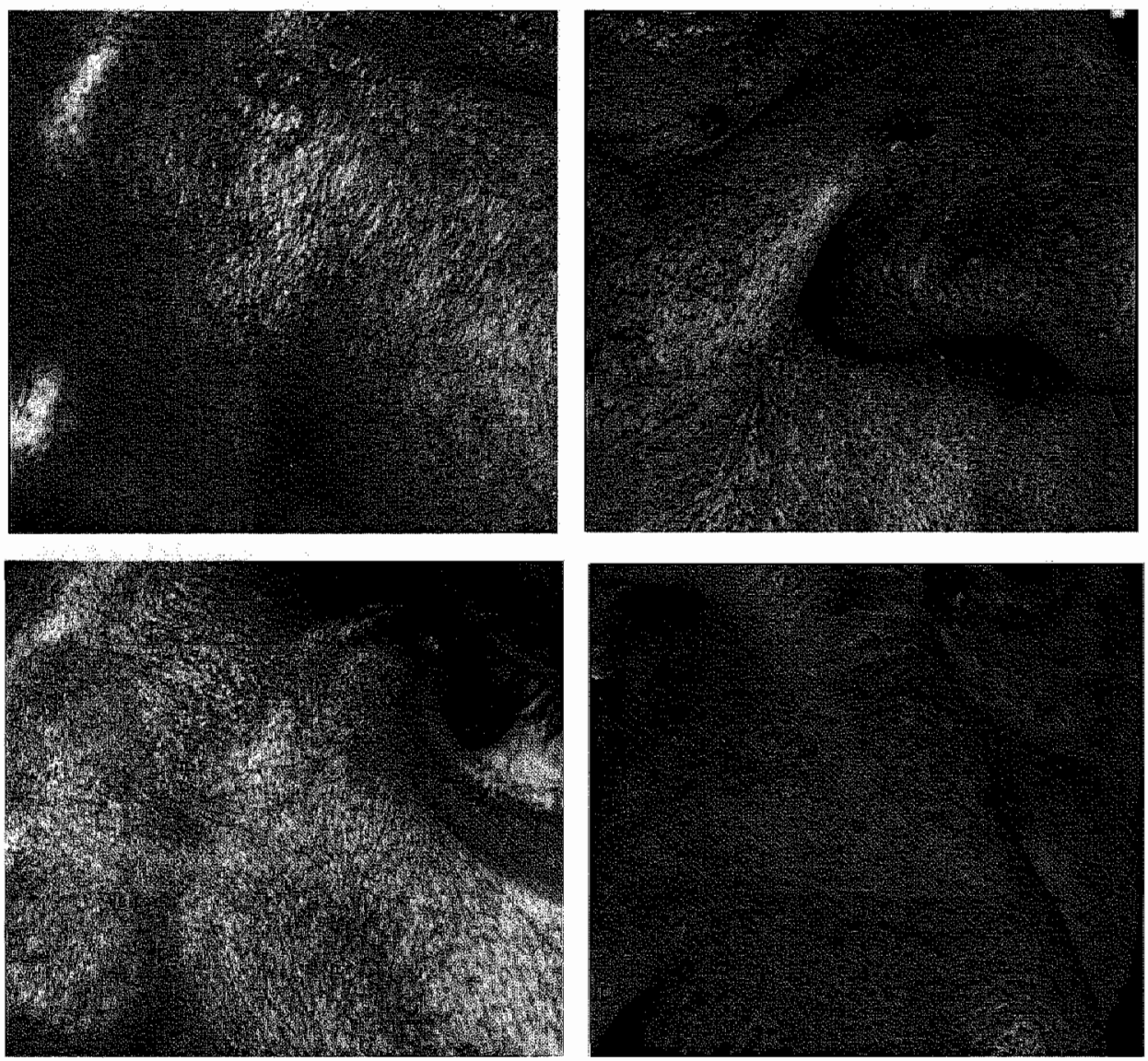

Figure 6.1

Primary Tumours before (up) and Clinical Response/Cosmetic Results (below) after topical 5-ALA/PDT, using a preceding debulking technique for nodular BCCs in the medial eye-angle (left) and on the lateral part of the nose (right). 
performed carefully, examining vertical sections every $0.1 \mathrm{~mm}$, it still remains possible, however, that residual nests could be missed (Abide, 1984). Further follow-up of the patients treated in this study will be necessary, for detection of long term recurrent $\mathrm{BCC}$.

The fact that either the photosensitizer or the light (or both) can not penetrate deep enough into the thicker tumour volume, could be the reasons why the nodular type shows less favorable response to PDT (Peng,1995; Morton, 1998). The penetration depth of the light depends for the greater part on the wavelength of the light beam. Wavelengths longer than $585 \mathrm{~nm}$ can theoretically penetrate the deeper layer of the dermis (Figure 2.3) (Goldman, 1994). The partial debulking of the tumour before PDT, which was not as deep and careful as the curettage in the combined curettage/electrodesiccation treatment, was performed to reduce the thickness of the tumour. Recently, Soler et al. (1999) reported the same complete clinical response rate of $92 \%$ at one year follow-up after PDT and preceding debulking in a larger series of patients with nodular BCCs. The combination of this partial debulking and the standardized 5-ALA application (only the desired treatment area is exposed to the sensitizer and the light), together with light reflecting occlusive dressings, thus avoiding degradation of the sensitizer by natural daylight (photobleaching), and the use of the standardized lightsource, could be the explanation for the good results in this study.
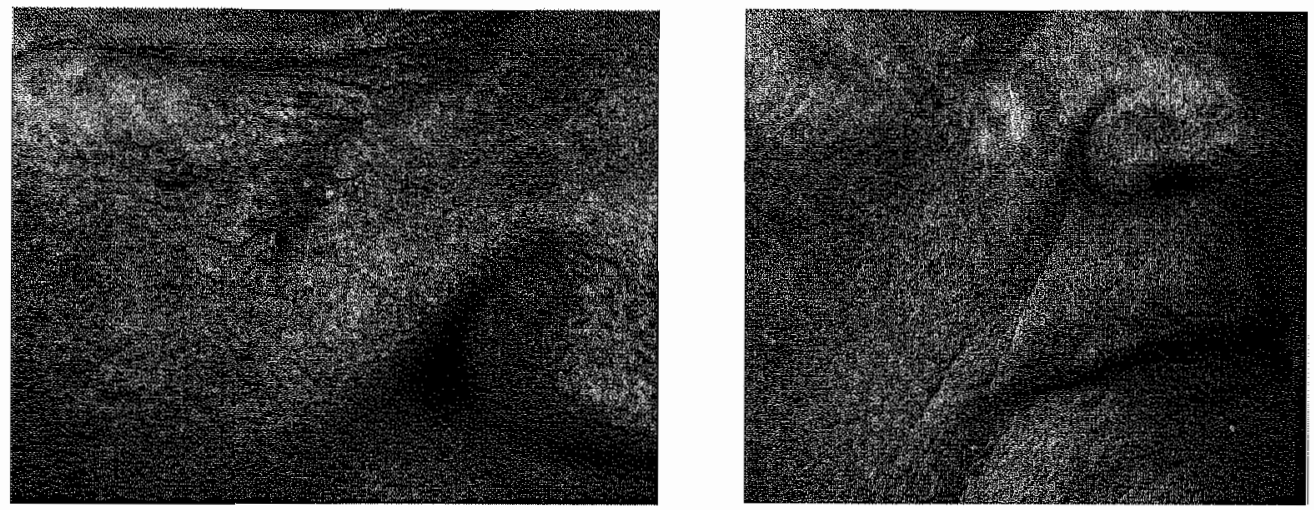

Figure 6.2

Primary Tumour before (left) and Cosmetic results after cryosurgery (right) for a nodular BCC on the cheek. 
Chapter Six

\section{CONCLUSIONS}

Taking into account the limited statistical power of $24 \mathrm{BCCs}$ treated, this study demonstrates that PDT with topically applied 5-ALA and the intensive VersaLight ${ }^{R}$ lightsource, and preceding partial debulking, can be an effective and safe method to treat nodular $\mathrm{BCCs}$, in cases where a nonsurgical, selective therapy for this malignant skin tumour is indicated. The complete clinical response rates in this study might be comparable with those of other established, non-surgical (non-sharp) therapies like cryosurgery, curettage/electrodesiccation, and radiotherapy. Cosmetic results on the other hand are better (Figure 6.1 and 6.2) and serious side effects were not observed. 


\section{Chapter Seven}

IN VIVO PHARMACOKINETICS OF PpIX ACCUMULATION FOLLOWING INTRACUTANEOUS INJECTION OF 5-AMINOLEVULINIC ACID FOR PHOTODYNAMIC THERAPY: investigation of dose-response relationship

$$
\text { A.W. de Blois }
$$

M.R.T.M. Thissen

D.J. Robinson

H.S. de Bruijn

R.J.E. Grouls

R.P. Dutrieux

H.A.M. Neumann 


\section{Abstract}

Background: Photodynamic therapy (PDT) with topically applied 5-aminolevulinic acid (5-ALA) is used successfully for superficial skin lesions. The results for nodular lesions are less favorable.

Objective: ALA-PDT is a high potential therapy for the increasing problem of skin cancer. The disadvantage of the topical administration route of ALA might be the insufficient penetration into deeper, nodular lesions. Therefore, intracutaneous administration of $A L A$, as an altemative administration route, was investigated to optimize this therapy.

Methods: The kinetics of the ALA-induced photosensitizer PpLX fluorescence after intracutaneous injection of 5-ALA, in different concentrations and different volumes, in normal porcine skin was measured, and compared to the $P p D X$ fluorescence kine tics after topical application of 20\% 5-ALA in cream, in order to investigate a dose/ response relationship. Both the visual and the histopatholagical PDT-induced skin damage after intracutaneous administration of the different ALA doses were investigated and compared with the damage after topical application of ALA.

Results: This study demonstrates, that intracutaneous injections of $3 \mathrm{mg}$ ALA or more, lead to a faster occurning and significantly higher $P p D X$-induced fluorescence levels, than those measured after topical application of ALA. The level of fluorescence after intracutaneous injections up to $10 \mathrm{mg}$ ALA, was shown to be dose dependent. The PpIX-induced fluorescence after topical application of $20 \% 5$-ALA cream was comparable with the fluorescence levels of a $2 \%$ injection of ALA. After injection of 2 mg ALA or more, the PDT-induced skin damage following irradiation with $60 \mathrm{~J} \mathrm{\textrm {cm } ^ { - 2 } /}$ $100 \mathrm{~mW} \mathrm{~cm}^{2}$, was significantly greater than after topical application of $20 \%$ 5-ALA. An injected dose of $10 \mathrm{mg}$ ALA $(0.5 \mathrm{ml}$ of a $2 \%$ solution/ resulted in significantly more severe and deeper localized damage than all other injected doses.

Conclusion: The results of this study justify further investigation of PDT with intracutaneous injection of ALA in human nodular BCC. 
In recent years 5-aminolevulinic acid (5-ALA) induced protoporphyrin IX (PpIX) has been used as a photosensitizing agent in photodynamic therapy (PDT). Effectiveness of this treatment has been demonstrated in a variety of malignant and non-malignant conditions, particularly in dermatology (Peng, 1997). Several investigators have examined the suitability of PDT with 5-ALA for the treatment of basal cell carcinomas (BCC). However, in nodular tumours, which are located deeper in the skin, complete clinical response rates have been much lower (Svanberg, 1994; Calzavara-Pinton,1995; Wolf, 1993). Insufficient penetration of 5-ALA into the tumour has been suggested as a possible explanation for this poor response. Although several methods have been used to enhance 5-ALA penetration, such as the addition of disodiumedetate and dimethylsulfoxide or prolonging the application time to between 12 and 24 hours, the variable uptake of 5-ALA caused disappointing PDT results (Peng,1995; Orenstein,1995; Szeimies,1994). Administration of 5-ALA via other routes might result in increased concentrations in tumours, especially in those localized deeper in the skin. Oral and intravenous administration of 5 ALA in humans, leading to accumulation of PpIX in several organs including the skin, has been described (Peng,1995; Loh, 1993). This, however, caused generalized side-effects, ranging from nausea and vomiting to more severe complications, like marked vasodilation combined with hypotension (Rick, 1997; Herman, 1998). Besides this, systemic administration of ALA will result in generalized hypersensitivity to (day)light of both the skin and the eyes, which is a serious drawback for this administration route. Local, intracutaneous injection of 5-ALA might increase the concentrations of PpIX in the tumour without these unwanted side-effects. A case report was published by FinkPuches et al. (1997), showing the result of PDT using intrallesional injection of 5-ALA and visible light in a patient with a large BCC on the back. Although in this case the response was promising, with complete disappearance of the tumour and no recurrence during a follow-up of 54 months, there has been no thorough investigation of this administration route. Casas et al. (1999) compared topical and intratumoural administration of 5-ALA in a subcutaneously implanted mammary adenocarcinoma in mice. They reported higher concentrations of PpLX after intratumoural injection, as well as delayed tumour growth following PDT.

This study investigates the kinetics of PpIX-induced fluorescence and 
the local PDT-induced tissue damage after intracutaneous administration of 5-ALA, compared to topical application, in order to examine the suitability of the intracutaneous injection as an alternative route for topical administration. Because intracutaneous administration of 5-ALA has not been used extensively in humans yet, the experiments were performed on the normal skin of pigs. Porcine skin resembles human skin best, both on histologic and physiologic aspects, although the dermal part of the porcine skin is thicker (Meyer, 1978 and 1996).

For intracutaneous injection of 5-ALA, it is necessary to prepare a stable and non-irritating solution. Aqueous solutions of 5-ALA are known to be chemically unstable, depending on the $\mathrm{pH}$ and the concentration of the solution (Novo,1992; Elfsson, 1998). In order to find a balance between stability, which demands a $\mathrm{pH}$ as low as possible, and the desirability to inject a nonirritating solution, which requires a pH between 4 and 9 , solutions with a $\mathrm{pH}$ of 5 were chosen to use in this study. Previously it was shown, that solutions of up to $2 \%$ with a pH of 5.0 are stable for at least 100 days (de Blois, submitted). So far, it is unknown which quantity of 5-ALA should be administered to obtain detectable PpIX fluorescence and moreover to obtain PDT-induced tissue damage. Therefore, different concentrations as well as different volumes were injected, allowing a possibility to investigate concentration- or dose dependency of PpIX fluorescence kinetics. After topical administration, usually a time interval of 4 to 8 hours between administration of 5-ALA and illumination is used [Cairnduff, 1994; Svanberg, 1994]. Because the penetration of ALA through the stratum corneum is bypassed in the injection route, an enhanced initial rate of increase of PpIX-induced fluorescence might be expected. The PDT-induced damage was assessed both histologically and visually, using a visual skin damage score. A number of spots have also been treated with 5-ALA injections without subsequent illumination, to investigate the toxicity of the injected solution without PDT.

\section{MATERIALS AND METHODS}

\section{Animal model}

All experiments were performed on the normal skin of 3 six-weeks-old female dutch pigs weighing $20 \mathrm{~kg}$ (Maastricht University, the Netherlands). After fasting overnight and premedication had been given, using azaperon 1 $\mathrm{ml} \mathrm{kg}^{-1}$ i.m. (StresnilR, Janssen-Cilag, the Netherlands), the animals were anesthetised with $2 \%$ halothane (Fluothane ${ }^{R}$, Zeneca, United Kingdom) in 
40-60\% $\mathrm{N}_{2} \mathrm{O}$ and oxygen. The hair on the back was removed by shaving. Between 28 and 60 circular test areas of $2 \mathrm{~cm}$ diameter were marked on the dorsal skin of each animal. To prevent diffusion of 5-ALA between adjacent test areas, the spots were positioned $5 \mathrm{~cm}$ apart. During the whole experiment, the pigs were placed on a temperature controlled blanket and remained anesthetised.

\section{Photosensitizing agent}

On six test areas designated for topical treatment, 5-ALA (hydrochloride, Merck, the Netherlands) was applied in the form of a freshly prepared $20 \%$ 5-ALA cream (w/o) (Schering AG, Germany). On control sites, only the vehicle was applied. To standardize the treated area and prevent spreading, the cream was applied within the gap of a synthetic ring that was stuck to the skin. Test areas designated for intracutaneous treatment, were injected at the centre of the site with an aqueous solution of 5-ALA in concentrations of $0.5,1.0$ or $2.0 \%$, each in volumes of $0.1,0.3$ or $0.5 \mathrm{ml}$, using a small needle $(0.5 \times 16 \mathrm{~mm}, 25 \mathrm{Gx} 5 / 8$ ", Sherwood Medical, Ireland) (Figure 7.1). Injections for each concentration/volume combination were performed sixfold on six different spots. The choice for these concentrations and volumes was based on a previous experiment, investigating the PpIX fluorescence following injections of $0.2 \mathrm{ml}$ of 0.0001 to $5 \%$ 5-ALA in a limited number of spots, leading to detectable PpIX fluorescence after injection of a solution containing $0.1 \% 5$-ALA or more. A volume of $0.5 \mathrm{ml}$ was shown to be the maximal volume that could be injected intracutaneously in this animal model. Solutions of 5-ALA were prepared by dissolving the appropriate amount of 5-ALA in water for injection, and adding $\mathrm{NaOH}$ to obtain a $\mathrm{pH}$ value of 5.0 . Starting from a demonstrated isotonicity of a $2 \%$ 5-ALA solution, at lower concentrations of 5-ALA sodium chloride was added in an appropriate amount to obtain an isotonic solution. All other solutions were prepared within 24 hours prior to use. Control sites were injected with $0.5 \mathrm{ml}$ of the solvent. Immediately after administration of 5-ALA, each test area was covered with aluminium foil and an ordinary dressing to protect the test areas from ambient light.

\section{Fluorescence kinetics}

Fluorescence was induced with low intensity 405 and $514 \mathrm{~nm}$ (bandwith $5 \mathrm{~nm}$ FWHM) using the output from a Xenon arc lamp and a monochro- 


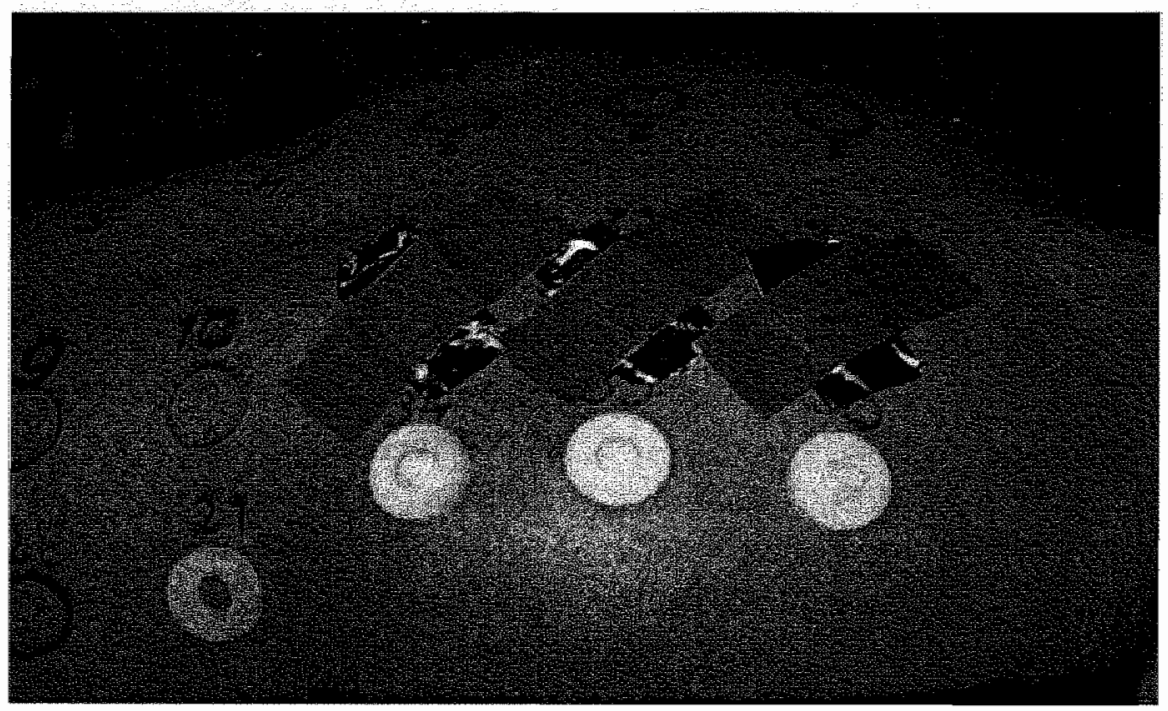

Figure 7.1 (a)

Topical application of 5-ALA cream.

A standardized amount of cream was applied by filling the entire gap of a synthetic ring that was stuck to the skin.

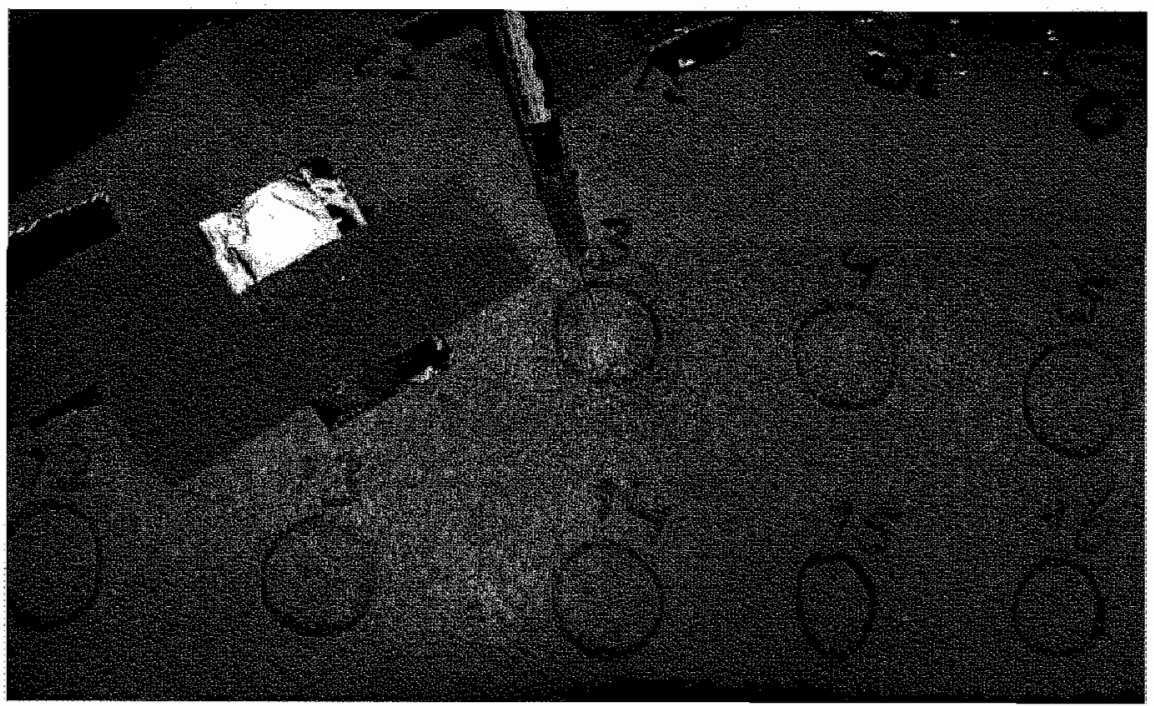

Figure 7.1 (b)

Intracutaneous injection of 5-ALA.

A maximum volume of $0.5 \mathrm{ml}$ could be injected intracutaneously, before the injected solution started to leak throughout the pores. 
mator system (models 60100 and 77250 respectively; Oriel Instruments Corporation, Stratford, CT, USA). Excitation light was delivered to the surface of the skin via a central 600 micrometer-core optical fibre. An outer ring of optical fibres was used to collect the emitted light, which was filtered loG 570 , long-pass) to block the scattered excitation radiation, and delivered to the entrance slit of a spectrograph and CCD camera (Oriel Instruments Corporation, Stratford, CT, USA). The delivery and detector fibres are arranged perpendicular to the skin surface in a hand-held probe, that analyses a circular area of appoximately $3 \mathrm{~mm}$, resulting in a localized irradiance of 250 microW $\mathrm{cm}^{-2}$. Each spectrum presented here was collected over a period of 5 seconds, resulting in a light fluence of $1.25 \mathrm{~mJ} \mathrm{~cm}{ }^{-2}$ delivered to the area of analysis during every measurement.

Prior to administration of 5-ALA, an emission spectrum of the autofluorescence from the centre of each test site was acquired under 405 and $514 \mathrm{~nm}$ excitation. Subsequent spectra were recorded in the same way from the centre of each test site at 3,5, 6.5 and 8 hours after intracutaneous injection of 5-ALA. After topical application of ALA, in each spot the cream was wiped off before measurement of PpIX fluorescence. To prevent disturbance of the normal 5-ALA uptake pattern in the skin by application of new cream layers, each measurement at $3,5,6.5$ and 8 hours was performed on a new spot. The spectra display the characteristic porphyrin signature. The emission at $636 \mathrm{~nm}$ was used to quantify the fluorescence from PpIX.

\section{PDT light delivery}

All test areas treated with PDT were irradiated exactly 3.5 hours after the administration of 5-ALA with broadband red light $(600-730 \mathrm{~nm})$ from a 1200 Watt filtered metal halogen lamp (Waldmann Medical Techniques, Munich, Germany). A diaphragm was used to restrict the irradiation beam to the defined $2 \mathrm{~cm}$ of the test area. The irradiance at the tissue surface in each test area was adjusted by varying the distance between the light source and the surface of the skin. Temperature measurements of the treated skin were made prior to, and immediately following each irradiation, using a thermocouple thermometer (Exacon, Roskilde, Denmark), placed at the centre of the illuminated area.

\section{Visual and histopathologic skin damage}

Visible PDT-induced skin damage to each test area was assessed with 
respect to erythema, induration and oedema on a five-point scale at 48 and 96 hours after irradiation $(0=$ no symptoms; $1=$ mild reaction inside the test area; $2=$ moderate reaction in the whole test area; $3=$ severe reaction outside the test area; and $4=e x t r e m e$ reaction with crust/necrosis outside the test area). The scores from each treatment site were used to determine a median skin score for each group. Visual skin damage was also assessed 7 days after PDT, in order to evaluate the healing process. At 48 and 96 hours after the end of the irradiation, full thickness punch-biopsies of $4 \mathrm{~mm}$ in diameter were taken from each test area, fixed in formaldehyde and embedded in paraffin. Sections of 5 micrometer thickness were examined microscopically for presence of necrosis, inflammatory cells and vascular changes. The maximum depth of PDT induced damage was determined in relation to the cutaneous cell layers.

\section{Statistics}

Measured fluorescence values were statistically compared using an analysis of variance (ANOVA) combined with a Student-Newman-Keuls test. The skin damage after PDT was statistically evaluated by a non-parametric Mann-Whitney $U$ test. For all tests, a $p$ value less than 0.05 was considered to be statistically significant.

\section{RESULTS}

\section{Fluorescence kinetics}

There was no significant difference between the kinetics of PpIX fluorescence intensity using 405 and $514 \mathrm{~nm}$ excitation light. All results presented have been acquired with $405 \mathrm{~nm}$ excitation light. Figure 7.2 shows the measured fluorescence excited at $405 \mathrm{~nm}$ at different concentrations of ALA for a constant injected volume. An increase in fluorescence intensity is observed when injecting higher concentrations of 5-ALA. A similar increase in fluorescence intensity is found following injection of larger volumes, while keeping the concentration of ALA constant, indicating a dose-dependent mechanism. This can be seen most clearly when injecting $0.5 \mathrm{ml}$ of different concentrations or injecting $2 \%$ in different volumes, where the absolute difference in dose is greatest. These findings are confirmed when the measured fluorescence is plotted directly against the injected dose (Figure 7.3). Until a dose of $6 \mathrm{mg}$ is reached, fluorescence values are increasing at a given time. A higher dose of $10 \mathrm{mg}$ does not lead to higher fluorescence values. For doses 

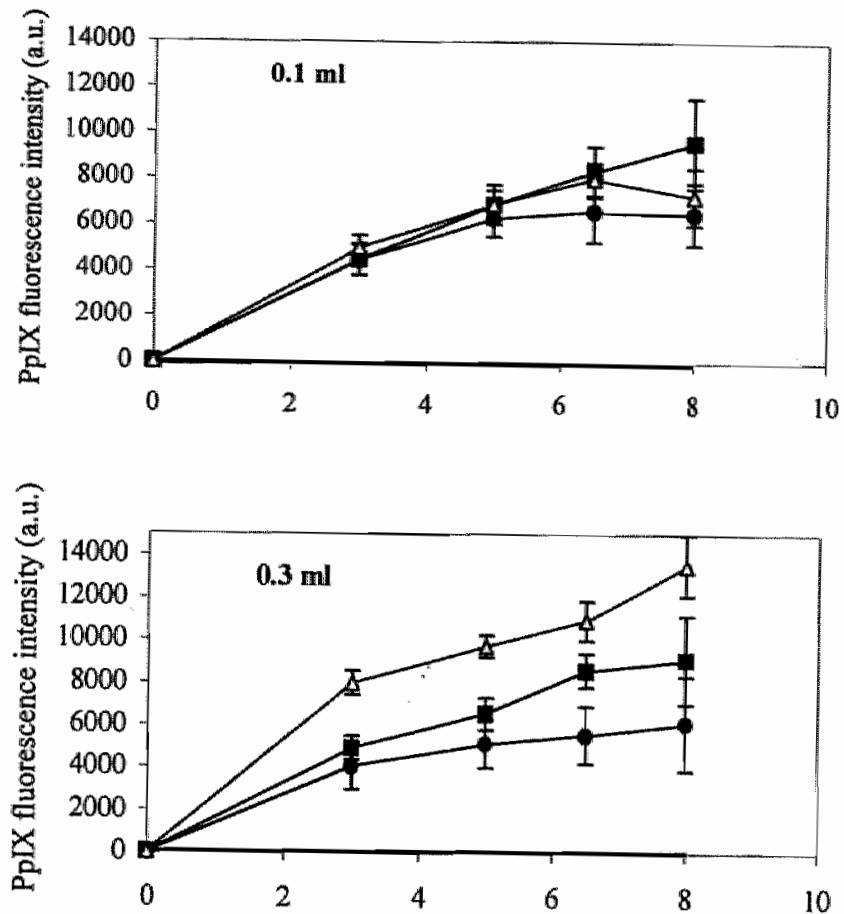

Concentrations

Alded.

- $\quad 0.5 \%$

a $1.0 \%$

$\triangle \quad 2.0 \%$

solvent

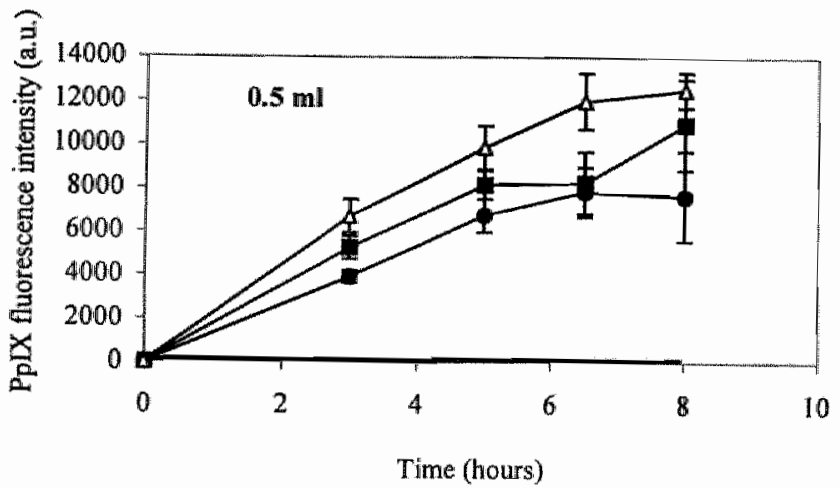

Figure 7.2

PpIX fluorescence at $405 \mathrm{~nm}$ after injections of $0.1,0.3$ and $0.5 \mathrm{ml}$ of ALA in concentrations of $0.5 \%, 1.0 \%$ and $2 \%(n=6$, mean $+1-S E M)$.

Higher fluorescence levels were observed when injecting higher volumes, as well as higher concentrations, indicating a dose dependent mechanism. 


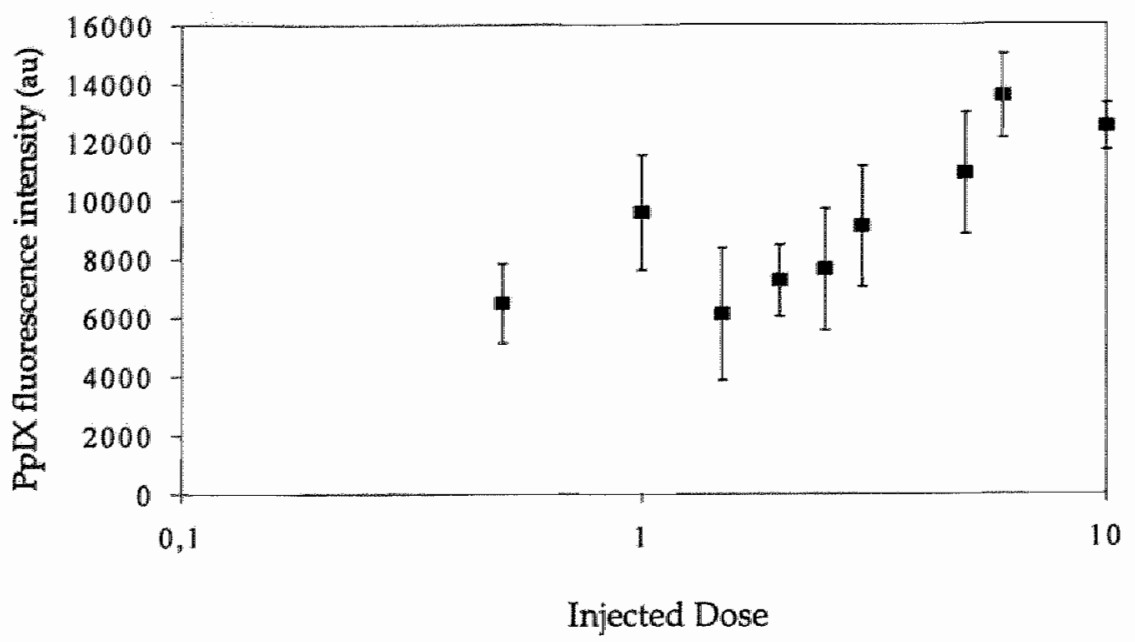

Figure 7.3

Injected Dose

Dose-fluorescence curve at 8 hours after intracutaneous injection of 5-ALA in doses of 0.5 to $10 \mathbf{~ m g ~ ( ~} \mathrm{n}=6$, mean $+/$ - SEM).

For injected doses between 1.5 and $6 \mathrm{mg} A L A$, a linear relationship ( $\left.r^{2=0} 0.9449\right)$ was shown. A higher dose of $10 \mathrm{mg}$ doesn't lead to further increase of PpIX fluorescence.

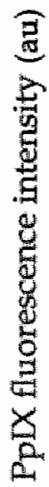

.

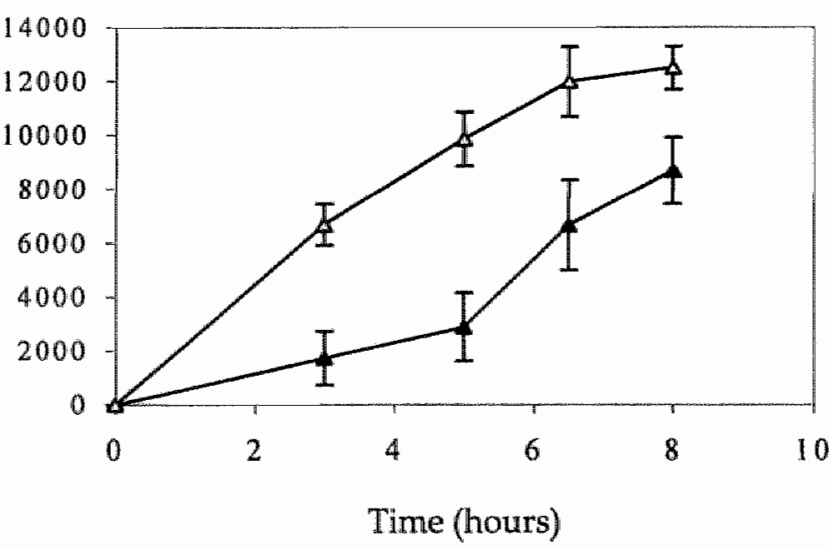

- Topical

$20 \%$ ALA

Intracutenous

$\triangle 0.5 \mathrm{ml} / 2 \%$ ALA

\section{Figure 7.4}

The PpIX fluorescence kinetics at $405 \mathrm{~nm}$ after topical and intracutaneous administration of 5-ALA ( $\mathrm{n}=6$, mean $+/$ - SEM). After injection of $A L A$, the PpIX fluorescence is increasing faster and reaches significantly higher values than after topical application. 
of $6 \mathrm{mg}$ and $10 \mathrm{mg}$, a statistically significant higher fluorescence level than for any other dose, was found at 6.5 and 8 hours after administration. Between these two doses there was no mutual significant difference.

All spots treated topically with $20 \% 5$-ALA cream showed fluorescence too, although the accumulation of PpIX developed much slower. In Figure 7.4 the fluorescence curve of topically applied 5-ALA is shown, together with the fluorescence curve obtained after injection of $0.5 \mathrm{ml}$ of a $2 \%$ solution of 5 ALA (dose $10 \mathrm{mg}$ ). Fluorescence values after injecting $10 \mathrm{mg}$ were significantly higher than after application of the cream for all time points measured. The fluorescence measured in the topically treated spots at 3 and 5 hours after application, was significantly lower than in any injected spot. After 6.5 and 8 hours, the fluorescence values following topical application of 5-ALA were comparable to injected doses of 2.5 and $3 \mathrm{mg}$ respectively. Although between 6 and 8 hours after injection fluorescence values stabilized at a certain level for most doses, an obvious decrease of fluorescence was not observed over the maximum observation time course ( 8 hours) of the experiment for any injected dose. Also after topical application of 5-ALA, no decrease of PpIX fluorescence was observed within the first 8 hours after application.

\section{PDT effect}

Injection of 5-ALA, in a concentration of 0.5 to $2 \%$, without irradiation did not cause any visible erythema or induration at 48 and 96 hours after administration. The visual PDT-induced skin damage was significantly greater with intracutaneous than with topical administration of 5-ALA. Figure 7.5 shows the median of six observations at 48 and 96 hours after PDT. All spots treated with injected or topical ALA showed a significantly higher damage score at 48 and 96 hours than the control spots treated with solvent or cream (without ALA). The test sites treated with topical 5-ALA cream showed a slight brownish discoloration of the skin with mild erythema inside the test site at 48 hours after irradiation, which persisted at 96 hours. After injection of 5-ALA, more severe damage was seen, which increased with higher injected doses. Injected doses of $2 \mathrm{mg}$ ALA or more showed a significantly higher damage score than topical ALA. At the highest injected dose of $10 \mathrm{mg}$, the area of visible damage (erythema, oedema and induration, as well as crust formation) even exceeded the test area. The damage score at this dose was significantly higher than at the all other injected doses or topical application 


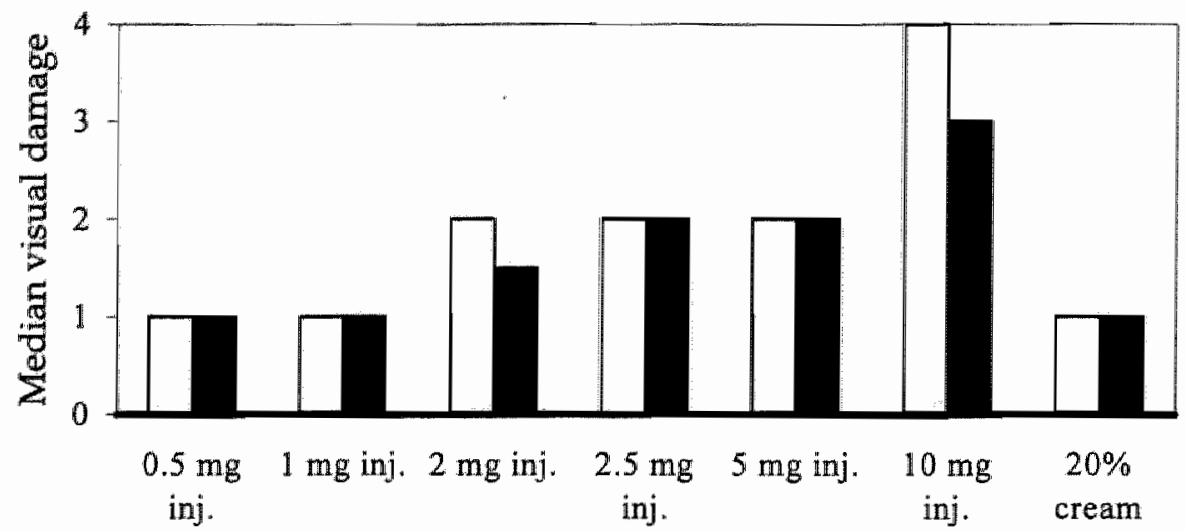

Administered ALA

Figure 7.5

PDT-induced visual damage score at ( $\square$ ) 48 and ( 0 ) 96 hours after intracutaneous injection of 0.5 to $10 \mathrm{mg}$ ALA or topical application of $20 \%$ ALA cream, followed by irradiation $\left(60 \mathrm{Jcm}^{-2} / 100 \mathrm{mWcm}^{-2}\right)$ after 3.5 hours.

A correlation between the injected dose of ALA and PDT-induced damage is shown. Injected doses of $2 \mathrm{mg}$ or more lead to significantly greater damage than topically applied ALA. Furthermore, a dose of $10 \mathrm{mg}$ injected ALA results in significantly greater damage than all other administered doses.

of ALA. In all cases, the visible damage at 96 hours was the same or less. After 7 days, all test areas had completely healed without atrophy or scars.

These visual observations are supported by histopathologic examination of full thickness punch biopsies at 96 hours after PDT (Table 7.1). After topical application of 5 ALA, focal necrosis was detected at the upper epidermal layer. In the other skin layers no damage was observed. Injection of 5ALA, followed by irradiation, resulted in damage localized deeper in the skin, increasing with higher 5-ALA doses. Doses under $5 \mathrm{mg}$ mainly led to necrosis of the epidermis and superficial dermis, and to inflammatory infiltrates around small blood vessels. Injection of 5 and $10 \mathrm{mg}$ resulted in signs of vasculitis and thrombosis of the larger dermal blood vessels. Some biopsies showed inflammatory cells in the subcutaneous layer and destruction of the hair follicles (Figure 7.6 . 
Table 7.1

Histological Changes observed 96 hours after PDT* with Intracutaneous and Topical Administration of Different Doses 5-ALA.

\section{ALA Dose}

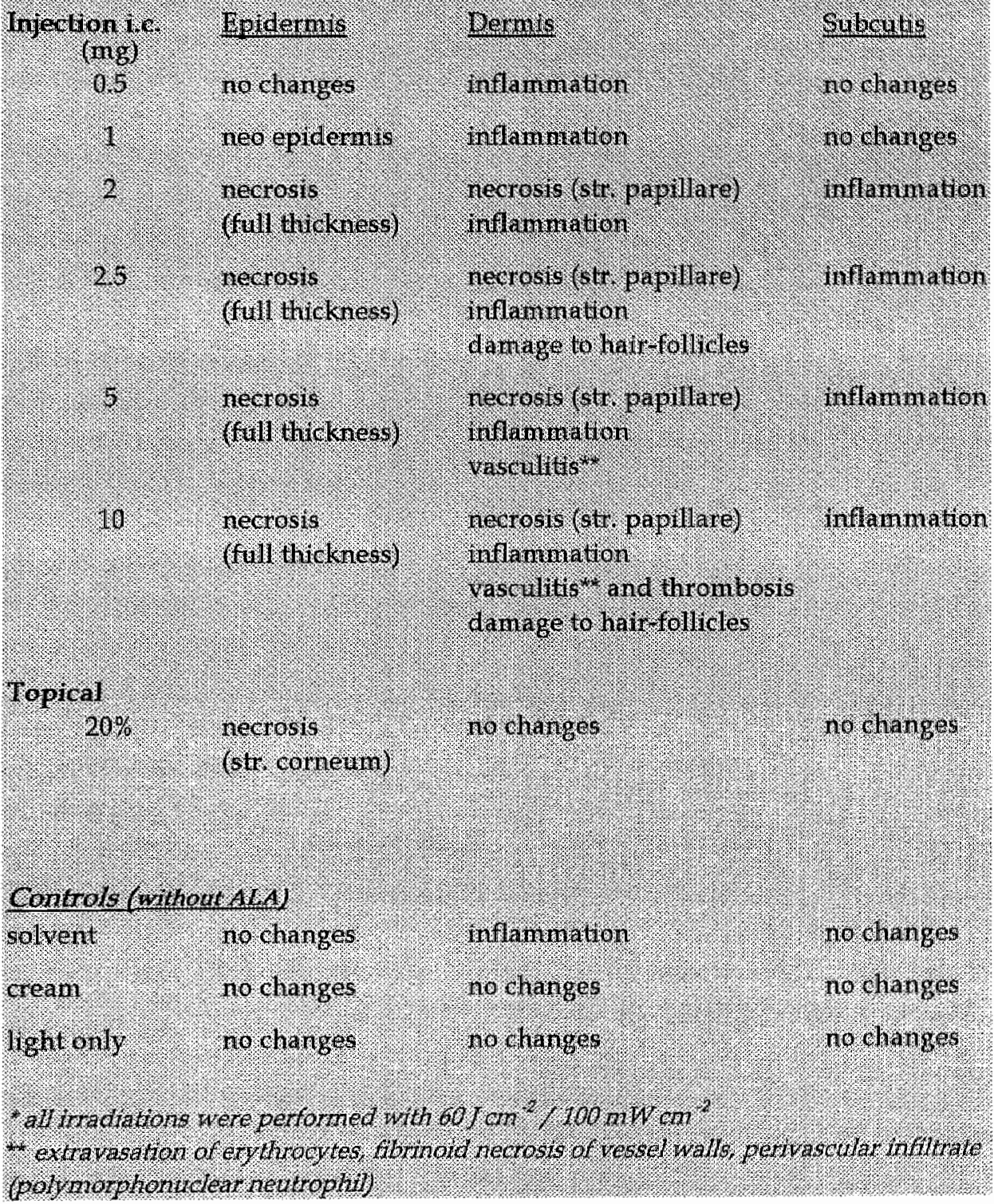



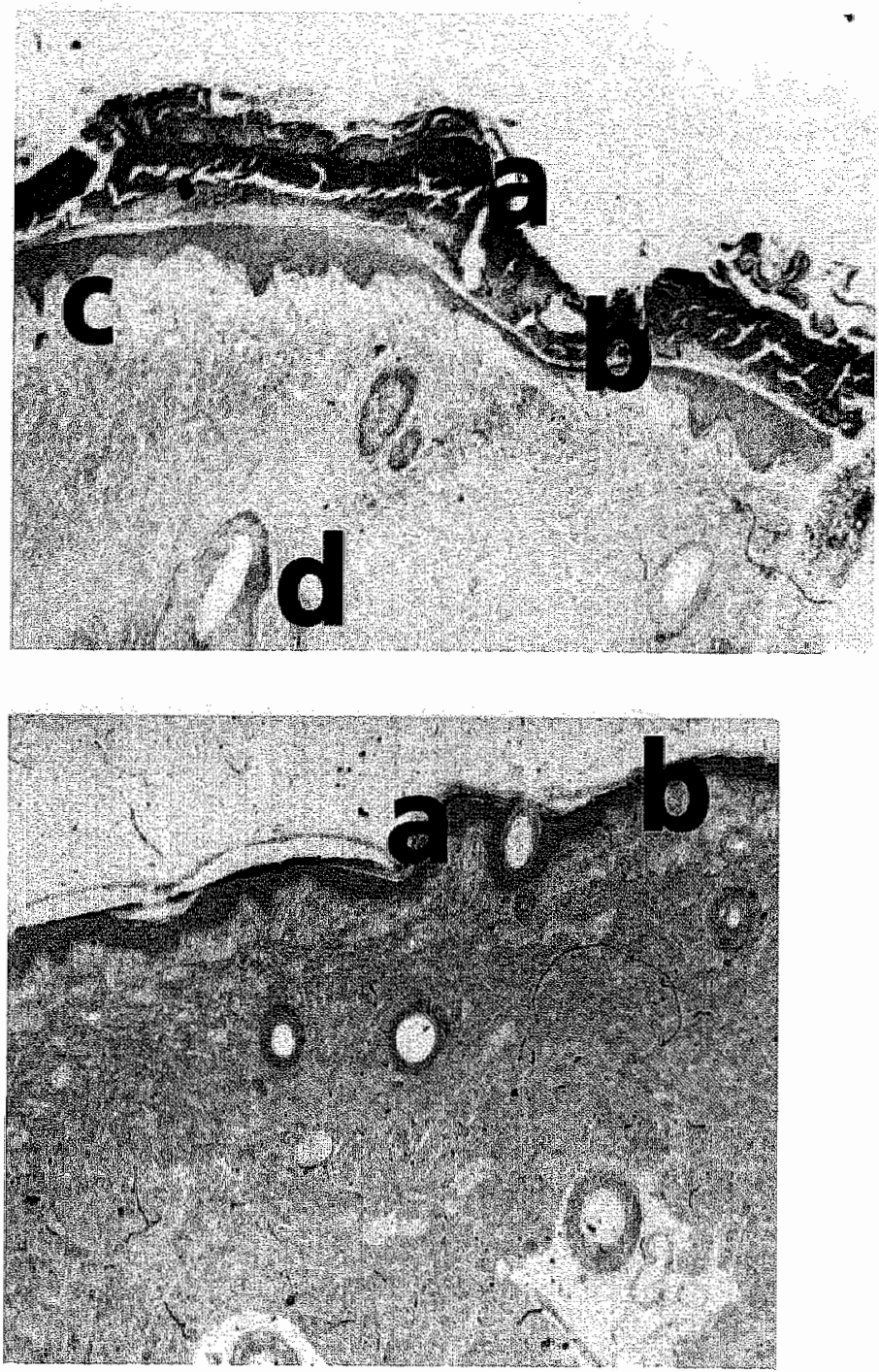

Figure 7.6

Histopathologic Changes at 96 hours after PDT $\left(60 \mathrm{Jcm}^{-2} / 100 \mathrm{mWcm}^{-2}\right)$ for Intracutaneously Injected solution of $2 \%$ 5-ALA (up) and Topically Applied 20 \% 5-ALA cream (below).

Damage after injection is more pronounced and deeper localized. The necrotic part of the epidermis/dermis (a) already has been shed and neo-epidermis (b) is formed. After injection/PDT, the upper dermis shows maderate edema (c) and inflammatory infittrate (d) in the deeper layer, whereas the dermis after topical cream/PDT shows no reaction. 


\section{COMMENT}

\section{Fluorescence kinetics}

Over the past decade, many studies have been published concerning the 5-ALA induced PpIX fluorescence kinetics after a variety of administration routes. Furthermore, in vitro experiments with cell lines have been performed to investigate the mechanism of PpIX generation (Iinuma,1994; Bech, 1997). In these experiments, cells incubated in solutions containing 5-ALA in a concentration range from $0.001 \%$ to $0.01 \%$ showed fluorescence. As the accessability of cells for ALA during in vitro incubation is much better than in vivo, the necessity of using higher concentrations for intracutaneous injection of ALA, to be able to measure any fluorescence, is plausible. From this study it becomes clear that a concentration of 0.5 to $2 \%$ provides the possibility to inject an amount of ALA that leads to detectable fluorescence values high enough to cause more severe PDT-induced damage than can be achieved after topical administration of ALA. Furthermore, it was demonstrated that injection of higher doses than $6 \mathrm{mg}(0.3 \mathrm{ml}$ of a $2 \%$ solution) does not lead to higher surface fluorescence values. Published data that include intracutaneous injection, used a concentration as high as $10 \%$ 5-ALA in $\mathrm{NaCl} 0.9 \%$, apparently without adjustment of the $\mathrm{pH}$ (Fink-Puches, 1997; Casas, 1999). A different reason to choose a maximum concentration of $2 \%$, apart from the mentioned lack of effectiveness gain when injecting higher concentrations, is that this concentration in water is iso-osmotic, and therefore causes minimal irritation and pain during injection. The $\mathrm{pH}$ value of an unbuffered solution of $10 \%$ 5-ALA is about 2. Apart from the pain this must cause upon injection, which has been reported earlier for intravenous 5-ALA injections (Loh, 1993), this low $\mathrm{pH}$ can delay PpIX formation and is therefore not advisable (Bech, 1997).

One of the theoretical advantages of injecting 5-ALA is the bypassing of the penetration step through the stratum corneum and thereby shortening the time interval between administration of ALA and illumination. In practice, these time intervals after topical administration of 5-ALA vary between 4 and 8 hours (Svanberg, 1994; Cairnduff 1994). This study shows that fluorescence values after injection of 5-ALA are increasing faster than after topical application (Figure 7.4), demonstrating that the injection is more effective in PpIX generation in all cases than the topically applied cream. Following injection of $0.5 \mathrm{ml}$ of a $2 \%$ solution, 3 hours later fluorescence values are reached comparable to those values found at 6.5 hours after topical ap- 


\section{Chapter Seven}

plication of 5-ALA. Although a few hours waiting time still have to be incorporated in treatment schemes, this means a substantial time gain when these data prove to be applicable to patients. In this way, intracutaneous injection of 5-ALA will facilitate the applicability of PDT. Theoretically, it will be difficult to shorten this time-interval even more, because this time will be necessary to convert 5-ALA into PpIX via enzymatic reactions, which seem to be the rate-limiting factors.

The dose dependence of PpIX accumulation, as demonstrated in this study, has also been observed by other investigators. Casas et al. (1999) have shown higher PpIX accumulation after increasing intratumorally injected doses, with a maximum at $30 \mathrm{mg}$ injected 5-ALA per $\mathrm{cm}^{3}$. Dose dependency has also been demonstrated by Rhodes et al. (1997), who showed a linear relationship between PpIX fluorescence and 5-ALA doses, corresponding to charges of 3-120 mC applied via iontophoresis. Our data show a linear relationship for 5-ALA doses between 1.5 and $6 \mathrm{mg}$ at 8 hours after injection $\left(\mathrm{r}^{2}=0.9449\right.$; Figure 7.3). Dose dependency of PpIX fluorescence is anticipated, as a higher injected dose leads to increased availability of 5-ALA for PpIX synthesis. After injection of doses of $0.5 \mathrm{mg}$ and $1.0 \mathrm{mg}$, however, measured fluorescence values were higher than expected on the basis of the data at higher injected doses. The reason for this event is unknown. The two lowest injected doses refer to the lowest used injection volumes of $0.1 \mathrm{ml}$. Perhaps there was less spreading of the injected solution in these test spots, leading to relatively higher amounts of 5-ALA available for conversion into $\mathrm{PpIX}$ in a smaller area, and consequently resulting in a higher fluorescence.

The highest dose of $10 \mathrm{mg}$ did not cause further accumulation of PplX, which possibly indicates that at this point saturation of enzymes involved in the synthesis of PpIX from ALA has occurred, and a balance between synthesis and disappearance of PpIX is reached. Saturation of PpIX synthesis has also been demonstrated in cell experiments (Bech,1997).

In a number of curves, the fluorescence seems to level out at 8 hours after 5-ALA administration, although a maximum was not found in any of the curves, probably due to prematurely ending of the observation period. In studies investigating fluorescence kinetics after 5-ALA administration, a maximum of fluorescence was reached between 4 and 8 hours after topical application (Peng, 1997; Klinteberg, 1999), while after oral and intravenous administration in humans maxima of fluorescence between 3 and 10 hours were reported (Rick, 1997; Loh, 1993). Furthermore, a correlation between adminis- 
tration of higher doses and appearance of higher, more sustained, and later occuring maxima of fluorescence, was demonstrated after iontophoresis (Rhodes, 1997). In our study, there seem to be maxima of at least 8 hours following injection of most doses. For some of the lower doses however $10.5 \mathrm{mg}$, $2 \mathrm{mg}$ and $2.5 \mathrm{mg}$ ), a maximum is possibly reached at 6.5 hours. This is consistent with the studies mentioned above, and indicates that after injection ALA is kept intracutaneously long enough to cause prolonged fluorescence.

\section{Irradiation effect}

PDT-induced skin damage after injection of $2 \mathrm{mg}$ 5-ALA or more is significantly greater than that found after topical application of $20 \% 5$-ALA cream. Although after topical application a fluorescence intensity comparable with that after an injection of $3 \mathrm{mg}$ was found after 8 hours, the visual PDT effect at 48 hours was equivalent to a $0.5 \mathrm{mg}$ injection and histological investigation of the topically treated skin reveals only a slight focal necrosis of the upper part of the epidermis, that does not correlate with any injection. The discrepancy between fluorescence levels and the visual skin damage score might be caused by the difference in location of 5-ALA. After injection, 5-ALA is probably located deeper in the skin. Because the fluorescence is measured superficially, the two treatment schemes seem to correlate, but due to the greater penetration depth of the treatment light (630 nm), the results after PDT are different. Using excitation wavelengths of 405 and 51.4 $\mathrm{nm}$, penetration depths of a few hundred micrometer are reached (Svaasand, 1996). As no difference was observed between fluorescence measurements at these two wavelengths, 5-ALA induced PpIX is probably located still deeper in the skin after intracutaneous injection. During irradiation, the light with a wavelength of $630 \mathrm{~nm}$ has a greater penetration depth of a few millimeters [Svaasand, 1996). Accumulation of 5-ALA induced PpIX in deeper skin layers after parental administration of 5-ALA, compared to the depth reached after topical application has been shown before (Peng, 1995).

Another reason for the minimal damage after topical application of 5 ALA could be the difficult penetration of 5-ALA through healthy pig skin. Warloe et al. (1992) have demonstrated this difficulty of 5-ALA penetration through the intact keratin layer of the skin, while 5-ALA diffuses more easily through the abnormal epidermis overlying the $\mathrm{BCC}$. Research in patients has to be performed to compare the response after PDT with topical application 
of 5-ALA on BCCs to the response obtained after intracutaneous injection of 5-ALA. Also the tumour selective localization of ALA induced PpIX after intracutaneous administration has to be investigated. Several investigators have already shown this tumour selectivity, which is mainly caused by reduced amounts of the enzyme ferrochelatase and reduced iron concentrations in tumour tissue, as well as a higher metabolism due to an increased activity of the enzyme porphobilinogen deaminase (Fritsch,1997; Langer, 1999).

A correlation between administered 5-ALA dose and photodamage was demonstrated after visual inspection, as well as after histological examination, which has been reported earlier (Rhodes, 1997; Gilaberte,1997). After injection of a dose of $10 \mathrm{mg}$ 5-ALA, damage has proceeded to the deep dermal level with inflammation, vasculitis and thrombosis of larger dermal bloodvessels. Svanberg et al. (1996) and Liu (1997) reported a vascular effect including thrombi, accompanied by a decreasing bloodflow in the tumour after PDT with intravenous administration of 5-ALA in rats with hepatic tumours. In this way, tumour cell death is enhanced by interruption of bloodflow.

\section{CONCLUSION}

Summarizing, this study showed dose dependent 5-ALA-induced PpIX fluorescence and PDT-induced damage after intracutaneous injection of 5ALA in a vivo model. It was demonstrated that PpIX fluorescence occurred faster and to a higher level after intracutaneous injection of 5-ALA compared to topical application. Furthermore, a significantly greater visual damage score was observed for intracutaneously administered 5-ALA. Further research in future will focus on comparing intracutaneous versus topical administration in a human $\mathrm{BCC}$ model.

\section{Acknowledgement}

The authors would like to thank Erbe Nederland B.V. for providing the light source used in this study, and the members of the Central Animal Institute, Maastricht University for their assistance with preparation, anesthesia and care of the animals used in this study.

DJ Robinson and HS de Bruijn were supported by the Dutch Cancer Society (project DDHK-98-1686). 


\title{
Chapter Eight
}

PpIX FLUORESCENCE KINETICS AND INCREASED DAMAGE AFTER INTRACUTANEOUS INJECTION OF 5-AMINOLEVULINIC ACID AND REPEATED ILLUIMINATION

\author{
M.R.T.M. Thissen \\ A.W. de Blois \\ D.J. Robinson \\ H.S. de Bruijn \\ R.P. Dutrieux \\ W.M. Star \\ H.A.M. Neumann
}

Submitted 


\begin{abstract}
Background: Photodynamic therapy (PDT) with topically applied 5-aminoleunlinic acid (5-ALA) is used successfully for superficial skin lesions. The results for nodular lesions are less favorable.
\end{abstract}

Objective: ALA-PDT is a promising therapy for the increasing problem of skin cancer. In a previous study, the effectiveness of PDT with intracutaneous injection of ALA was demonstrated. The methods of ALA administration and the irradiation schemes are still under investigation, to optimize this therapy.

Methods: Both visual and histopathologic PDT-induced skin damage after intracutaneous administration of ALA was investigated in normal pig skin, with the intention to improve the lang term response rate of PDT for nodular basal cell carcinoma (BCC), where ALA penetration may limit the effectiveness of the therapy. The kinetics of the ALA-induced photosensitizer PpLX fluorescence after irradiation in relation to fluence and irradiance was also investigated. Finally, the effect of a fractionated irradiation on PDT-induced damage was observed.

Results: This study demonstrates again that intracutaneous administration of ALA leads to formation of $P p D X$ in higher concentrations compared with topical application of ALA-cream. The peak level of $P p D X$ after intra-cutaneous administration of ALA is reached earlier than after topical administration. The re-synthesis of $P p D X$ after irradiation is inhibited with increasing fiuence. PDT induced damage increases with increasing fluence, but is independent of the irradiance (50 us $100 \mathrm{~mW} \mathrm{~cm} \mathrm{~m}^{2}$ ). Finally, the PDT induced skin damage is significantly greater after repeated irradiations with two equal light fractions of $15 \mathrm{~J} \mathrm{cm^{-2 }}$ separated by a dark interval of 2 hours.

Conclusion: The results of this study justify further investigation of intracutaneous injected ALA-PDT with 2-fold fractionated irradiation in human nodular BCC. 
The response after photodynamic therapy (PDT) as a treatment for basal cell carcinoma $(\mathrm{BCC})$ mainly depends on the histologic subtype of this tumour. A complete initial clearance of more than $90 \%$ has been reported for treatment of superficial BCCs, but for thicker lesions such as nodular BCCs a complete response obtained with topical ALA-PDT is much lower [Wolf, 1990; Cairnduff,1994; Svanberg,1994; Calzavara-Pinton, 1995; Meijnders, 1996). An explanation for this lower efficacy might be insufficient penetration into the deeper layers of the tumour of either topically applied ALA or the light. Systemic administration (oral or i.v.) of ALA may improve the biodistribution of PpIX (Loh, 1993a) but will result in generalized hypersensitivity to daylight. Another method of improving the PpIX accumulation, and therefore the final effectiveness of PDT in nodular tumours, might be the intralesional injection of ALA, as already described previously in chapter seven.

On the other hand, a number of animal studies have demonstrated that the response to PDT can also be improved by modifying the irradiation scheme, for example by reducing the fluence rate or using light fractionation schemes. This may improve the oxygenation of the illuminated tissue (Hua, 1995; Robinson, 1998 and 1999) and will theoretically lead to the generation of more singlet oxygen, enhancing the response to therapy. (Pogue,1997). Another option is the use of long term fractionation, where two light fractions are separated by an interval of 1 hour or longer (de Bruijn, 1999). After the first light fraction PpIX is partially or completely photobleached, and in the time post irradiation, new PpIX is formed which can be used for a second irradiation. There is probably a variety of mechanisms involved in the enhancement of PDT response using a second light fraction.

In this study, performed on healthy porcine skin (Meyer, 1978 and 1996), the effects of both intracutaneous administration of ALA and repeated irradiations at different irradiances on the final PDT-induced tissue damage were investigated and compared with topical application of ALA and single irradiations, to find out whether the first technique may lead to improvement of the effectiveness of PDT. Finally, the kinetics of ALA-induced PplX fluorescence before and after irradiation were observed. The fluorescence level may indirectly give an indication about the effect of PDT that might be expected. Measurement of fluorescence was used to find the time-point of the 


\section{Chapter Eight}

maximum PpIX concentration, which may theoretically be the best moment for irradiation.

\section{MATERIALS AND METHODS}

\section{Animal Model}

All experiments were performed on the normal skin of six-week-old female dutch pigs weighing $20 \mathrm{~kg}$ (Maastricht University, the Netherlands). After fasting overnight and premedication had been given with azaperon $1 \mathrm{ml}$ $\mathrm{kg}^{-1}$ i.m. (Stresnil ${ }^{\mathrm{k}}$, Janssen-Cilag, the Netherlands) the animals were anesthetised with $2 \%$ halothane (Fluothane ${ }^{R}$, Zeneca, United Kingdom), 40-60\% $\mathrm{N}_{2} \mathrm{O}$ and oxygen. Between 25 and 35 circular test areas of $2 \mathrm{~cm}$ diameter were marked on the dorsal skin of each animal. To prevent diffusion of ALA between adjacent test areas, these areas were positioned $5 \mathrm{~cm}$ apart. Based on ALA dose response studies (de Blois, submitted), $2 \%$ 5-ALA (hydrochloride, Merck, the Netherlands) was dissolved in water and brought to a $\mathrm{pH}$ of 5.0 with $\mathrm{NaOH}$. This solution was injected intracutaneously in a volume of 0.5 $\mathrm{ml}$ at the centre of each test site. On another six test sites, $20 \%$ 5-ALA cream (w/o) was applied under occlusive dressing.

\section{Fluorescence kinetics}

Fluorescence was induced with low intensity 405 and $514 \mathrm{~nm}$ light (bandwidth $5 \mathrm{~nm}$ FWHM) using the output from an Xenon arc lamp and monochromator system (models 60100 and 77250 respectively; Oriel Instruments Corporation, Stratford, CT). Excitation light was delivered to the surface of the skin via a central 600 micrometer-core optical fibre. An outer ring of optical fibres was used to collect the emitted light, which was filtered (OG 570 , long-pass) to block the scattered excitation radiation and delivered to the entrance slit of a spectrograph and CCD camera (Oriel Instruments Corporation, Stratford, CT). The delivery and detector fibres were arranged perpendicular to the skin surface in a hand-held probe that analyses a circular area of approximately $3 \mathrm{~mm}$ in diameter, resulting in a localized irradiance of 250 microW cm$~^{-2}$. Each spectrum presented here was collected over a period of 5 seconds. This results in a light fluence of $1.25 \mathrm{~mJ} \mathrm{~cm} \mathrm{~cm}^{-2}$ delivered to the area of analysis during every measurement.

Prior to the administration of ALA, an emission spectrum of the autofluorescence from the centre of each test site was acquired under 405 and $514 \mathrm{~nm}$ excitation. Subsequent spectra were recorded in the same manner 
from the centre of each test site immediately prior to irradiation $3.5 \mathrm{~h}$ after the application of ALA, immediately post irradiation and then at 30 minute intervals until $2 \mathrm{~h}$ after irradiation with schemes described below. The spectra display the characteristic porphyrin signature with three lorentzian peaks deconvolved in each spectrum. The emission at $636 \mathrm{~nm}$ was used to quantify the fluorescence from PpIX, whilst a band centred at $674 \mathrm{~nm}$, characteristic of the hydroxy-aldehyde chlorin photoproduct, was also observed.

\section{PDT light delivery and irradiation schemes}

All test areas were irradiated $3.5 \mathrm{~h}$ after subcutaneous administration of ALA with broadband red light $(600-730 \mathrm{~nm})$ from a $1200 \mathrm{~W}$ filtered metal halogen lamp (Waldmann Medical Techniques, Munich, Germany). A diaphragm was used to restrict the irradiation beam to the defined $2 \mathrm{~cm}$ diameter test area. The irradiance at the tissue surface of each test area was adjusted by varying the distance between the light source and the surface of the skin. During irradiation each pig was placed on a temperature controlled blanket and remained anesthetised with a combination of $2 \%$ halothane (Fluothane ${ }^{\mathrm{R}}$, Zeneca, United Kingdom), $\mathrm{N}_{2} \mathrm{O}$ and oxygen. Continuous rectal and superficial skin temperature measurements were made prior to and immediately following each treatment using a thermocouple thermometer (Exacon, Roskildle, Denmark), placed at the centre of the irradiation area.

Results are presented from two groups of experiments: (i) In order to determine the kinetics of PpIX fluorescence and the tissue response after PDT, spectra were acquired prior to and following irradiation, $3.5 \mathrm{~h}$ after the administration of ALA, at $50 \mathrm{~mW} \mathrm{~cm} \mathrm{~cm}^{-2}$ to a total light fluence of $3,6,9,15$ and $30 \mathrm{~J} \mathrm{~cm}^{-2}$ ( $\mathrm{n}=6$ test sites in each group). Control sites, to which solvent (without ALA) only or $20 \%$ ALA cream (w/o) had been administered, were irradiated with a fluence of $30 \mathrm{~J} \mathrm{~cm}^{-2}(\mathrm{n}=4)$. Also, test sites were treated with light only $\left(30 \mathrm{~J} \mathrm{~cm}^{-2}\right)$. In order to investigate if any fluence rate effects existed, two series of test sites ( $\mathrm{n}=6$, in each) were irradiated at $100 \mathrm{~mW} \mathrm{~cm} \mathrm{~cm}^{-2}$ to a total light fluence of 6 and $30 \mathrm{~J} \mathrm{~cm}^{-2}$ respectively. (ii) The kinetics of PpLX fluorescence and tissue response after PDT were investigated in a series of two-fold irradiation schemes where various light fluences were delivered in two fractions separated by a dark intervall of $2 \mathrm{~h}$ (i.e. irradiation 3.5 and 5.5 $h$ after the administration of ALA) at an irradiance of $50 \mathrm{~mW} \mathrm{~cm}^{-2}$. Fluorescence spectra were acquired immediately prior to and following each 
irradiation, and at 30 minute intervals between irradiations. Again, control sites to which only solvent had been administered, were irradiated using a two-fold irradiation scheme ( $\mathrm{n}=4$, in each). In addition, the mechanism of distribution of ALA was investigated by monitoring the kinetics of PpLX fluorescence at two sites; one immediately adjacent to a test site that had received ALA, and one at a distance $(>20 \mathrm{~cm})$ from all other test sites $(n=2)$.

\section{Visual skin damage and histopathology.}

Visible skin damage to each irradiated area was assessed with respect to erythema, induration and oedema on a five-point scale at 48 and 96 hours after irradiation $(0=$ no symptoms; $1=$ mild reaction inside the test area; $2=$ moderate reaction in the whole test area; $3=$ severe reaction outside the test area; and $4=$ extreme reaction with crusts/necrosis outside the test area) by two independent observers. The scores from each treatment site were used to determine a median skin score for each group. Forty eight and 96 hours after the end of irradiation full thickness biopsies, $4 \mathrm{~mm}$ in diameter, were taken from each test area, first fixed in formaldehyde and subsequently embedded in paraffin. Five-micrometer slices were examined for the presence of necrosis, inflammatory cells and vascular changes. The maximum depth of PDT induced damage was determined with relation to the cutaneous cell layers. Visual skin damage to each test site was also assessed 7 days after PDT, evaluating the healing process.

\section{Statistics}

All data concerning PpIX fluorescence presented are the mean (error bars: $+/$ - SEM). The relative significance of these data was compared statistically using the analysis of variance (ANOVA), and if necessary, followed by a Student-Newman-Keuls test. Skin damage after PDT was statistically evaluated by a non-parametric Mann-Whitney $U$ test, resulting in a median skin damage score. For all tests a $P$ value of less than 0.05 was considered to be statistically significant.

\section{RESULTS}

\section{Intracutaneous administration of ALA leads to the formation of PpIX.}

Three and a half hours after intracutaneous administration of $2 \%$ ALA there is a significant increase in the PpIX fluorescence intensity at the centre of the test sites compared to the tissue autofluorescence before administration 


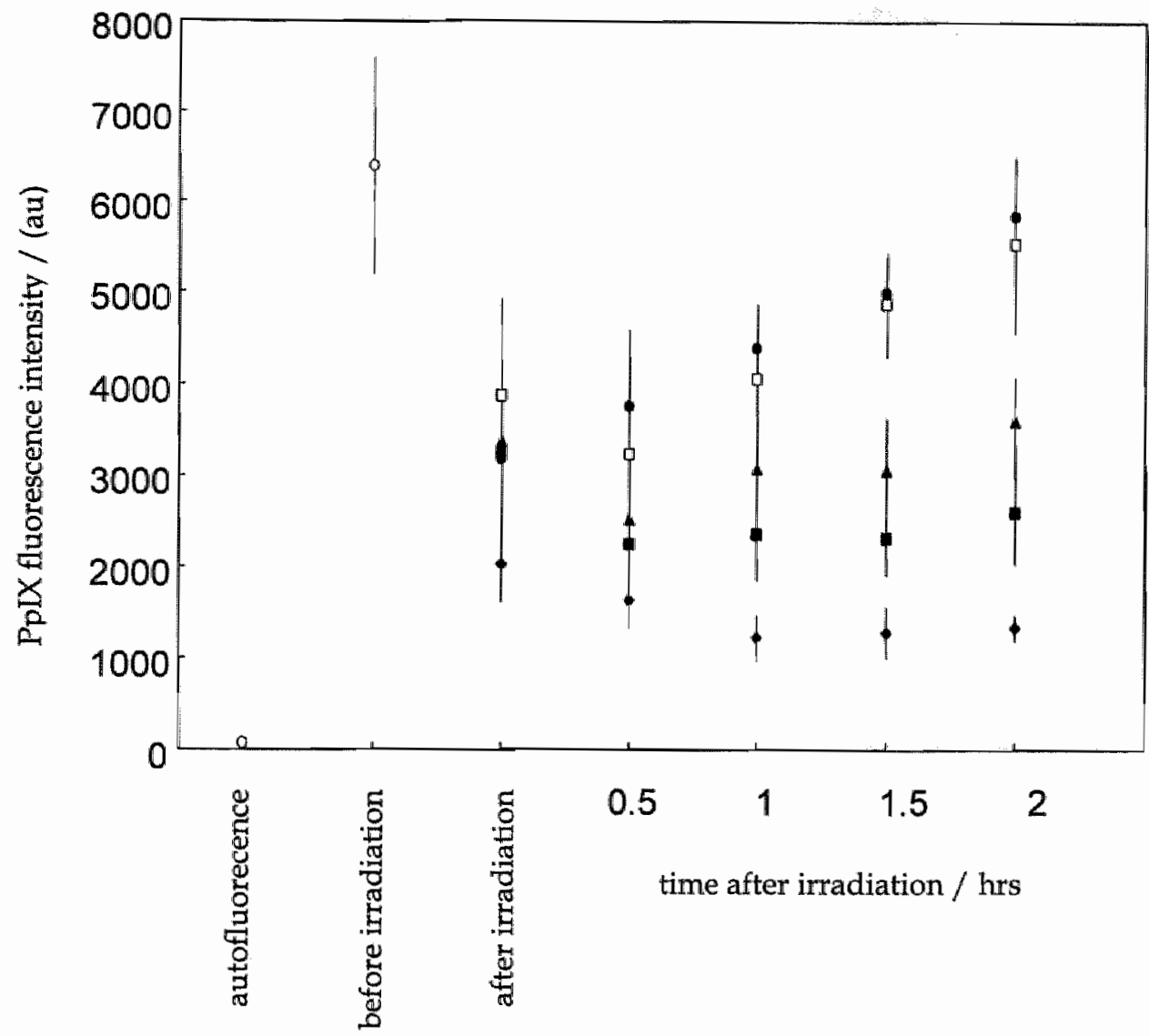

Figure 8.1

The PpIX fluorescence intensity measured, before the intracutaneous administration of ALA (tissue autofluorescence), immediately before irradiation $3.5 \mathrm{~h}$ after administration of ALA $(0)$, and after irradiation with $(\bullet \mid 3,1 \square) 6,(4) 9,(\square) 15$ and $(\bullet) 30 \mathrm{~J} \mathrm{~cm}^{-2}$ at $50 \mathbf{m W} \mathbf{c m}^{-2}(\mathrm{n}=6$, mean $+1-\mathrm{SEM})$.

Re-synthesis of $P p L X$ is dependent on the fluence delivered after 3 and $6 J_{\mathrm{cm}}^{2}$. The intensity of $P p D X$ fuorescence increases after photobleaching during irradiation. With increasing fiuence the re-synthesis of PpIX is strongly inhibited. 
of ALA. PpIX fluorescence intensity after intracutaneous administration of 2 $\%$ ALA is significantly greater than after topical application of $20 \%$ ALA. There is no significant difference in PpIX fluorescence intensity immediately before irradiation in any of the test sites investigated in this study. During irradiation at $50 \mathrm{~mW} \mathrm{~cm} \mathrm{~cm}^{-2}$, PpIX is rapidly photobleached. The intensity of PpIX fluorescence that remains immediately after irradiation with $50 \mathrm{~mW}$ $\mathrm{cm}^{-2}$ decreases with increasing fluence delivered during irradiation; there is significantly more photobleaching after $30 \mathrm{~J} \mathrm{~cm}^{-2}$, than after $3 \mathrm{~J} \mathrm{~cm}^{-2}$ and 6 $\mathrm{J} \mathrm{cm}^{-2}$ (Figure 8.1). There was no significant difference observed in the extent of PpIX photobleaching following irradiation with 50 and $100 \mathrm{~mW} \mathrm{~cm}^{-2}$, when equal fluences were delivered during irradiation.

\section{The re-synthesis of PpIX after irradiation is dependent on the fluence}

delivered. During the first $2 \mathrm{~h}$ after irradiation the re-synthesis of PpIX is dependent on the fluence delivered during irradiation. Following 3 and $6 \mathrm{~J}$ $\mathrm{cm}^{-2}$ the intensity of PpIX fluorescence increases significantly after irradiation and has almost returned to its pre-treatment intensity $2 \mathrm{~h}$ after the end of irradiation. With increasing fluence, the re-synthesis of PpIX is inhibited; for fluences above $15 \mathrm{~J} \mathrm{~cm}^{-2}$ the PpIX fluorescence intensity does not increase significantly for any of the time points investigated. Following $30 \mathrm{~J}$ $\mathrm{cm}^{-2}$ the intensity of fluorescence decreases, however, this is not statistically significant. (Figure 8.1). There was no significant difference between the kinetics of PpIX re-synthesis following irradiation at 50 and $100 \mathrm{~mW} \mathrm{~cm}{ }^{-2}$ when equal fluences were delivered during irradiation.

\section{PDT induced damage increases with increasing fluence up to $30 \mathrm{~J} \mathrm{~cm}^{-2}$} delivered in a single light fraction. The histologic and the visual damage observed at 48 and $96 \mathrm{~h}$ after PDT increases with increasing fluence (Figure $8.2,3,4 ;$ Table 8.1 ). Irradiation at $50 \mathrm{~mW} \mathrm{~cm}^{-2}$ to a fluence of 3 and $6 \mathrm{~J} \mathrm{~cm}^{-2}$ induced minimal redness $48 \mathrm{~h}$ after irradiation that resolved $96 \mathrm{~h}$ after irradiation. Increasing the fluence to $15 \mathrm{~J} \mathrm{~cm}^{-2}$ induced moderate redness and induration. After $30 \mathrm{~J} \mathrm{~cm}^{-2}$ we observed severe redness and induration at $48 \mathrm{~h}$ after irradiation, that was still present at $96 \mathrm{~h}$ after irradiation. Biopsies taken $96 \mathrm{~h}$ after irradiation demonstrated minimal histologic changes following 3 and $6 \mathrm{~J} \mathrm{~cm}^{-2}$; only dermal perivascular inflammation was seen and necrosis was absent or restricted to the stratum corneum after $6 \mathrm{~J} \mathrm{~cm}^{-2}$. Increasing the fluence to $30 \mathrm{~J} \mathrm{~cm}^{-2}$ resulted in necrosis of the up- 


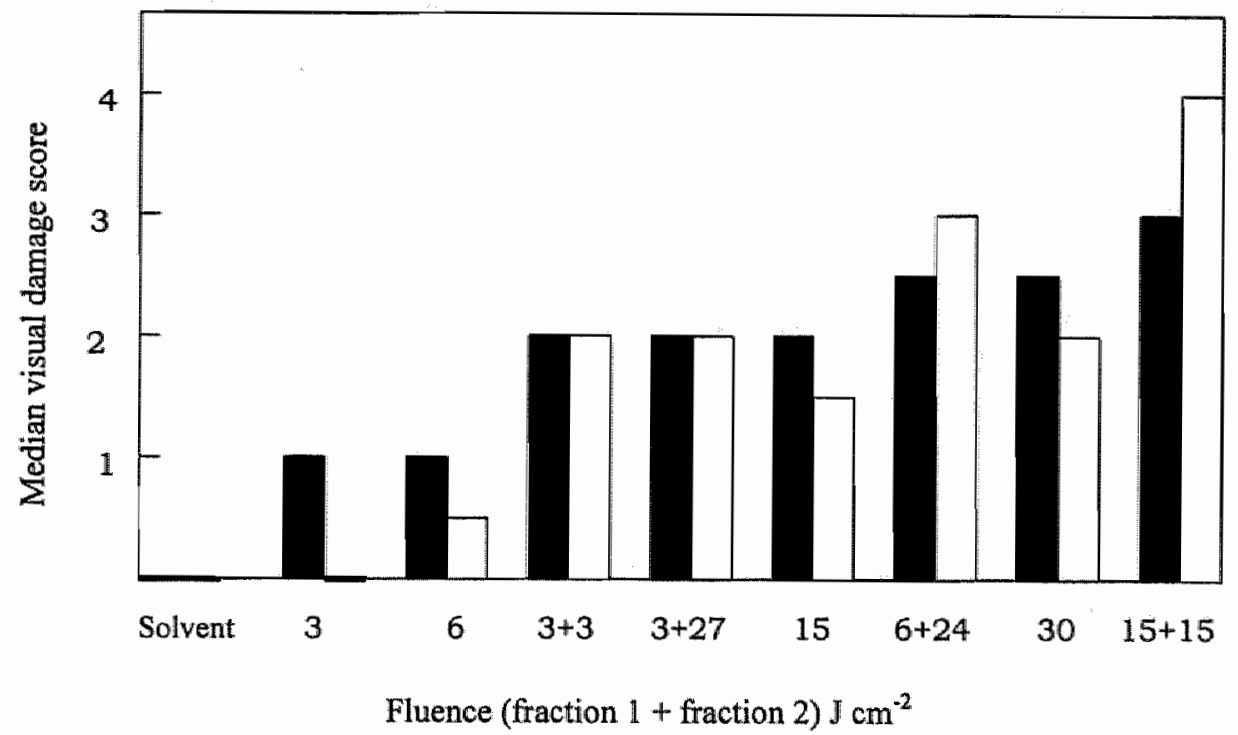

Figure 8.2

The Visual Skin Damage score ( $148 \mathrm{~h}$ and $(\square) 96 \mathrm{~h}$ after irradiation using a Variety of Single and Two-fold irradiation.

There is significantly more damage when the fluence is delivered in two fractions separated by an interval of $2 \mathrm{~h}$, than when an equal fluence is delivered in a single light fraction.

per third of the epidermis, vasculitis and inflammatory reaction in the dermis and the subcutis (Fig 8.4; Table 8.1). Biopsies taken at $48 \mathrm{~h}$ after irradiation demonstrated similar histologic changes to those described previously although oedema and necrosis were slightly more pronounced and the inflammatory infiltrate was more of an acute type (neutrophils and eosinophils rather than lymfocytes and histiocytes). Test sites injected with solvent did not show any significant response after single or 2 -fold irradiation with any of the schemes investigated. Also, the maximum temperature rise measured at the centre of each test site, was less than $3^{\circ} \mathrm{C}$ and the temperature had returned to that before irradiation within 2 minutes in all test sites. 


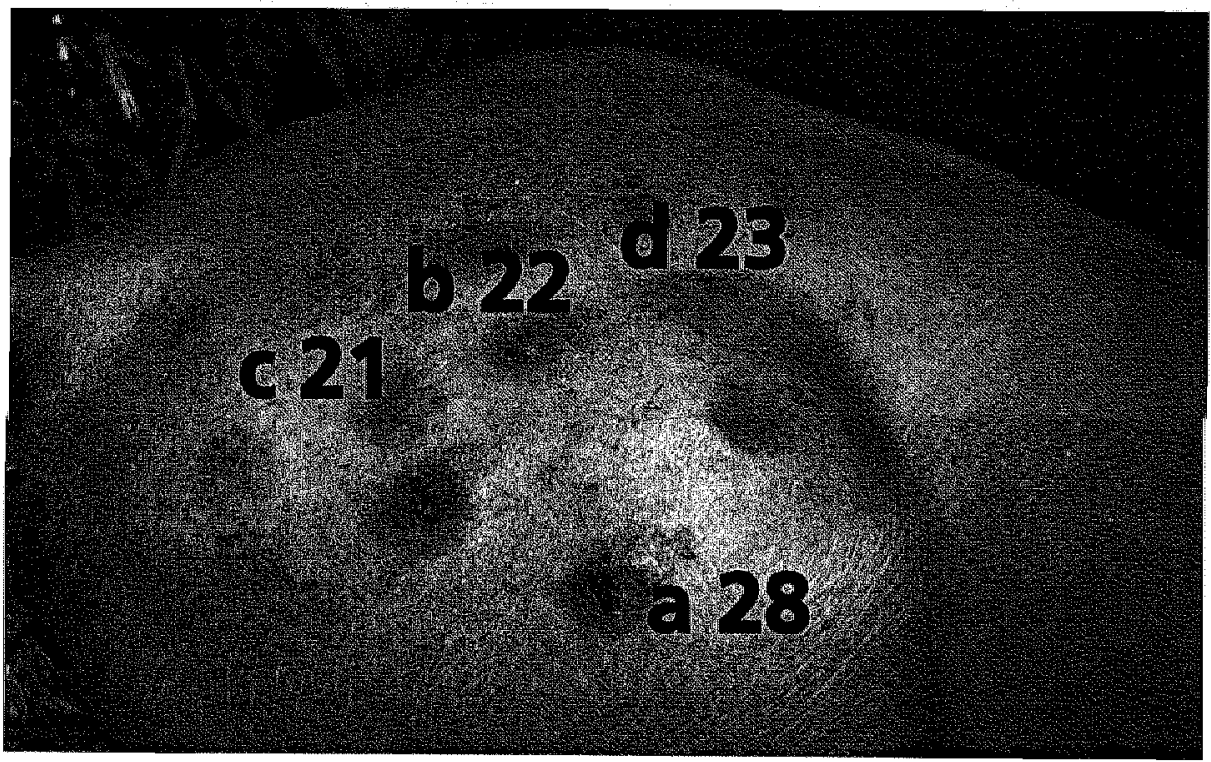

Figure 8.3

The Visual Skin Damage at $48 \mathrm{~h}$ (up) and at $96 \mathrm{~h}$ (below) after irradiation, using a Variety of Single and Two-fold Irradiation Schemes.

Damage after two-fold irradiation $\left(\boldsymbol{a}, 15+15 \mathrm{Jcm}^{2} ; \mathbf{b}, 6+24 \mathrm{Jcm}^{2}\right)$ is more severe than after single irradiation (c, $15 \mathrm{Jcm}^{2} ; \boldsymbol{d}, 30 \mathrm{Jcm} \mathrm{cm}^{2}$ ). 
PDT induced damage does not increase significantly when the irradiance is reduced from 100 to $50 \mathrm{~mW} \mathrm{~cm}^{-2}$. There was no difference observed in both the visual skin score and the histopathologic changes at 48

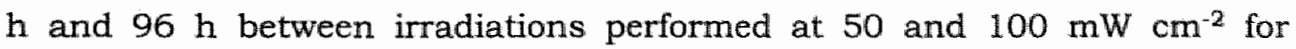
fluences of 6 and $30 \mathrm{~J} \mathrm{~cm}^{-2}$.

\section{PDT induced damage is significantly greater when the fluence is} delivered in two equal fractions separated by an interval of $2 \mathrm{hrs}$. The histopathologic and visual damage observed at both 48 and $96 \mathrm{~h}$ after PDT is significantly greater when a 2 -fold irradiation scheme is used, where equal fluence fractions are separated by a dark interval of $2 \mathrm{~h}(3.5$ and $5.5 \mathrm{~h}$ after the administration of ALA), compared with the damage observed after the same fluence is delivered in one fraction $3.5 \mathrm{~h}$ after the administration of ALA (Figure 8.2,3). This effect was observed for cumulative fluences of both 6 $\mathrm{J} \mathrm{cm}^{-2}\left(3+3 \mathrm{~J} \mathrm{~cm}^{-2}\right)$ and $30 \mathrm{~J} \mathrm{~cm}^{-2}\left(15+15 \mathrm{~J} \mathrm{~cm}^{-2}\right)$. Delivering $6 \mathrm{~J} \mathrm{~cm}^{-2}$ in a 2-fold irradiation scheme $\left(3+3 \mathrm{~J} \mathrm{~cm}^{-2}\right)$ results in moderate redness at both $48 \mathrm{~h}$ and $96 \mathrm{~h}$ after irradiation. At $48 \mathrm{~h}$ the damage is greater, but not significantly so, than that observed when $6 \mathrm{~J} \mathrm{~cm}^{-2}$ is delivered in a single fraction. The difference in skin damage is significantly greater $96 \mathrm{~h}$ after irradiation using the 2-fold irradiation scheme. Similarly, delivering $30 \mathrm{~J} \mathrm{~cm}^{-2}$ in a 2fold irradiation scheme $\left(15+15 \mathrm{~J} \mathrm{~cm}^{-2}\right)$ results in moderate and severe redness at $48 \mathrm{~h}$ and $96 \mathrm{~h}$ after irradiation respectively which is greater than that observed when $30 \mathrm{~J} \mathrm{~cm}^{-2}$ is delivered in a single fraction. Again the difference in skin damage is significantly greater $96 \mathrm{~h}$ after irradiation but not $48 \mathrm{~h}$ after irradiation using the 2-fold irradiation scheme.

Histopathologic changes observed $96 \mathrm{~h}$ after irradiation were also more severe when a 2 -fold irradiation scheme was used (Figure 8.4). Necrosis of the most superficial third of the epidermis is observed when $6 \mathrm{~J} \mathrm{~cm}^{-2}$ is delivered in a 2 -fold irradiation scheme $\left(3+3 \mathrm{~J} \mathrm{~cm}^{-2}\right)$, whereas necrosis is confined to the stratum corneum when $6 \mathrm{~J} \mathrm{~cm}^{-2}$ is delivered in a single fraction. Full-thickness epidermal necrosis, necrosis of the papillary dermis, oedema and inflammation in the subcutis are observed when $30 \mathrm{~J} \mathrm{~cm}^{-2}$ is delivered in a 2 -fold irradiation scheme $\left(15+15 \mathrm{~J} \mathrm{~cm}^{-2}\right)$, whereas necrosis is confined to the most superficial third of the epidermis when $30 \mathrm{~J} \mathrm{~cm}^{-2}$ is delivered in a single fraction. 
Table 8.1

Histological Changes observed at 96 hours after PDT for Different Fluences at $50 \mathrm{~mW} \mathrm{~cm}^{-2}$.

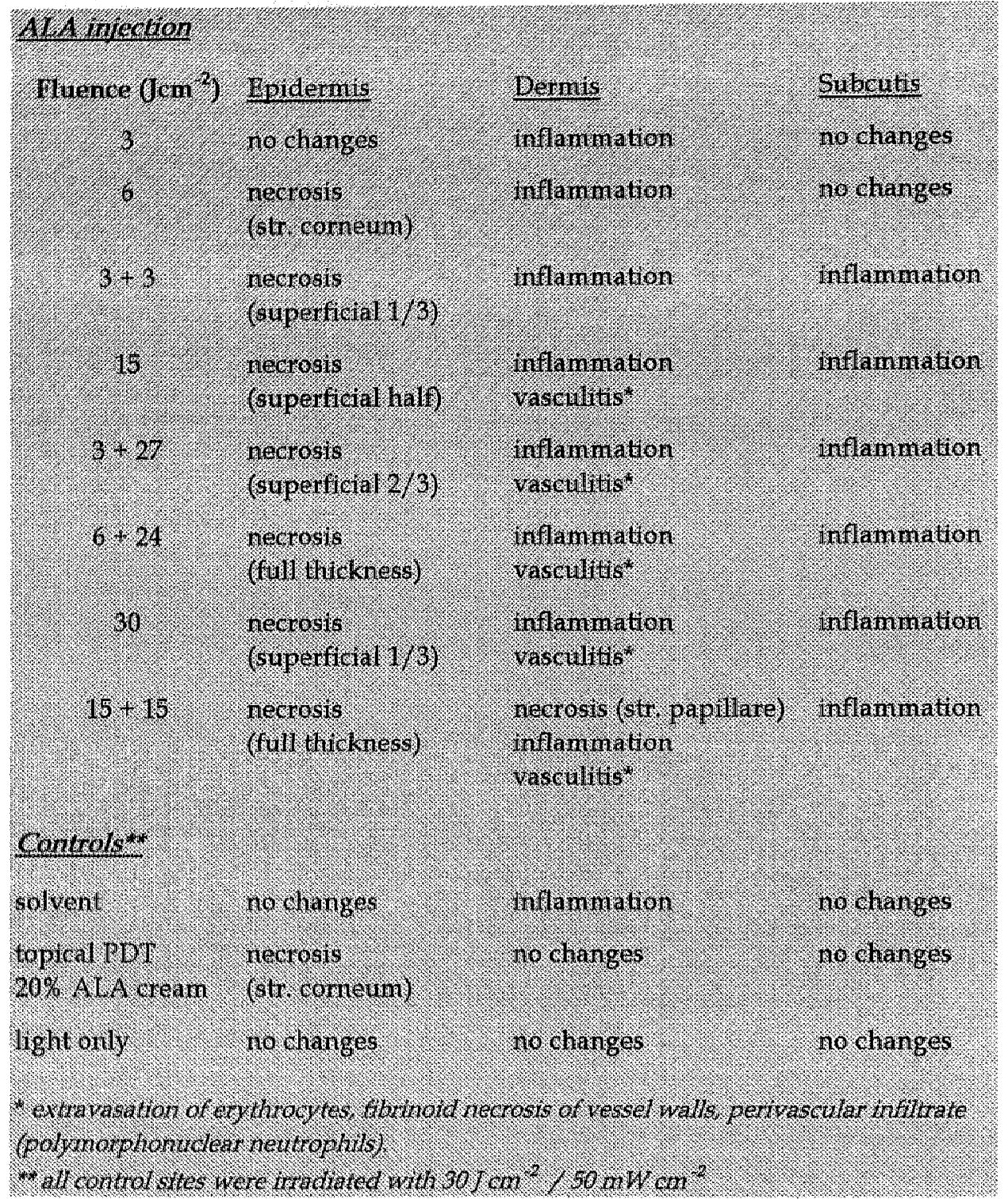



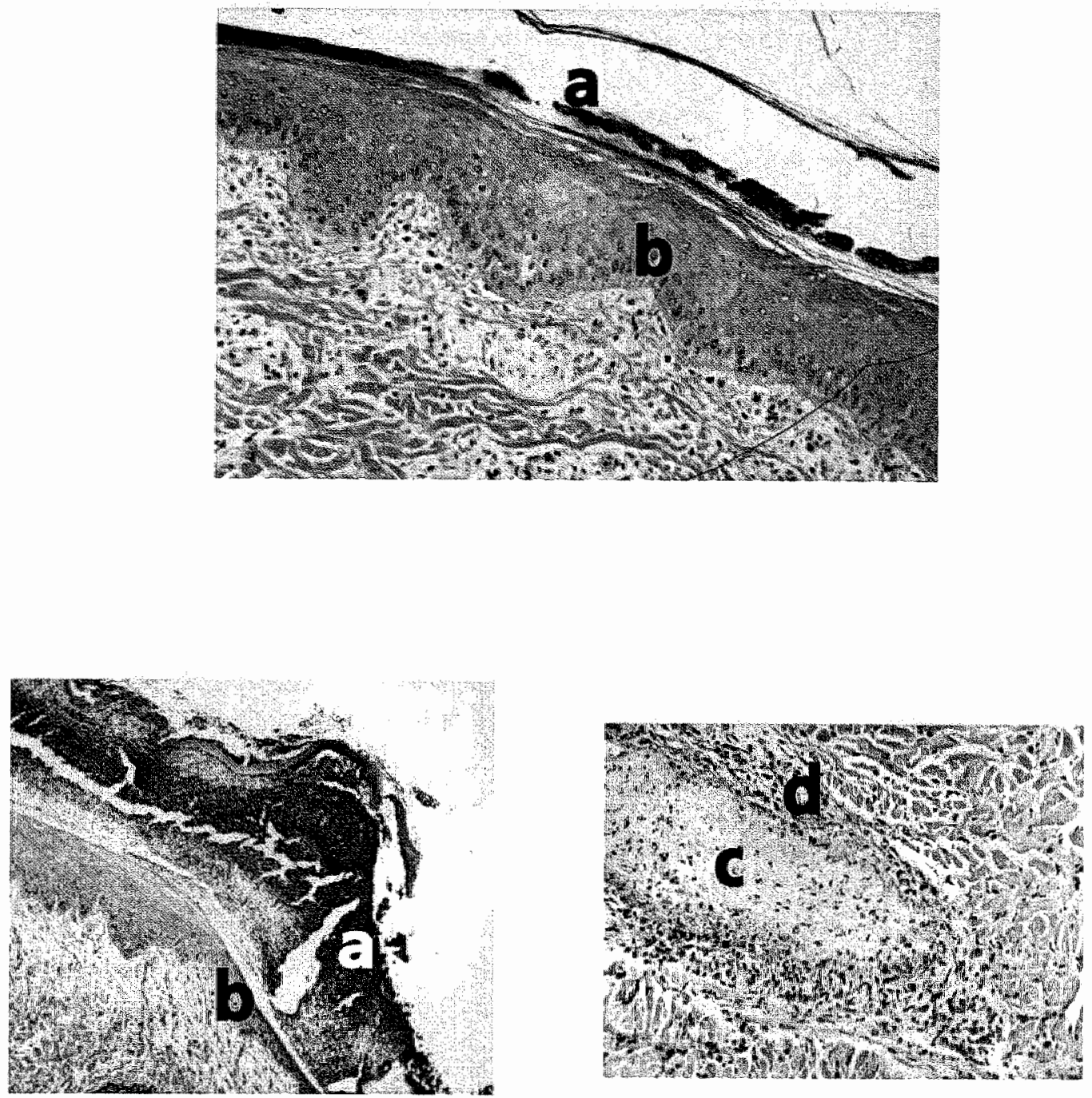

Figure 8.4

Histopathologic Changes at $96 \mathrm{~h}$ after Single (30 $\left.\mathrm{Jcm}^{-2}\right)$ (up) and Twofold $\left(15+15 \mathrm{Jcm}^{-2}\right)$ (below) irradiation at $50 \mathrm{mWcm}^{-2}$.

Damage after two-fold irradiation is more pronounced and deeper localized. The necrotic part of the epidermis/dermis (a) has already been shed and neomepidermis is formed (b). In the dermal layer complete vascular thrombosis (c) and signs of vasculitis (d) are observed. 
Delivering a low initial fluence followed by a second light fraction $2 \mathrm{~h}$ later does not significantly affect PDT response. The most effective treatment scheme investigated is $15+15 \mathrm{~J} \mathrm{~cm}^{-2}$. There was no significant increase observed in the histopathologic response or visual damage score at 48 and $96 \mathrm{~h}$ (Figure 8.2) using an irradiation scheme in which $30 \mathrm{~J} \mathrm{~cm}^{-2}$ was delivered using a 2-fold irradiation scheme that utilises the significantly enhanced PpIX fluorescence intensity immediately prior to the delivery of the second light fraction, $2 \mathrm{~h}$ after the first irradiation with both $3 \mathrm{~J} \mathrm{~cm}^{-2}(+27 \mathrm{~J}$ $\left.\mathrm{cm}^{-2}\right)$ and $6 \mathrm{~J} \mathrm{~cm}^{-2}\left(+24 \mathrm{~J} \mathrm{~cm}^{-2}\right)$ (Figure 8.1). Indeed, delivering $30 \mathrm{~J} \mathrm{~cm}^{-2}(15$ $+15 \mathrm{~J} \mathrm{~cm}^{-2}$ ) where the PpIX fluorescence immediately prior to the second light fraction is significantly lower, resulted in a significantly greater PDT response at $96 \mathrm{~h}$ after irradiation.

The photobleaching of PpIX is less during the second light fraction when two equal light fractions are delivered. During the second light fraction of the 2-fold irradiation scheme, in which the same fluences are delivered in each light fraction, the percentage of PpIX photobleaching is relatively less compared to the amount photobleached during the first irradiation; $[57.7 \%+/-10.9 / 40.7 \%+/-5.3]$ following $15+15 \mathrm{~J} \mathrm{~cm}^{-2}$ respectively and $[43.2 \%+/-13.4 / 32.8 \%+/-13.8]$ following $3+3 \mathrm{~J} \mathrm{~cm}^{-2}$ respectively. The difference between photobleaching in the first and the second light fraction is statistically significant following $15+15 \mathrm{Jcm}^{-2}$.

PDT induced skin damage completely heals in one week without formation of scars. Seven days after PDT none of the irradiated areas did show any sign of atrophy or scarring. The areas treated with $15+15 \mathrm{~J} \mathrm{~cm}^{-2}$ showed slight hyperpigmentation.

The administration of intracutaneous ALA results in the accumulation of PpIX at distant sites. The administration of intracutaneous $0.5 \mathrm{ml}$ of 2 $\%$ ALA to a large number of test sites in a single animal $(n=30$, total ALA dose $15 \mathrm{mg} \mathrm{kg}^{-1}$ ) results in the widespread accumulation of PpIX. Both a site distant $(>20 \mathrm{~cm})$ from those that received intracutaneous ALA and a site between two test areas show low PplX fluorescence intensities (approximately $12 \%$ of that of an injected site) with nearly equal fluorescence kinetics. Since the fluorescence increases at the same rate at the two sites (Figure 8.5), ALA or PpIX are probably not transported to adjacent sites via 
diffusion through porcine skin, but by a systemic route.

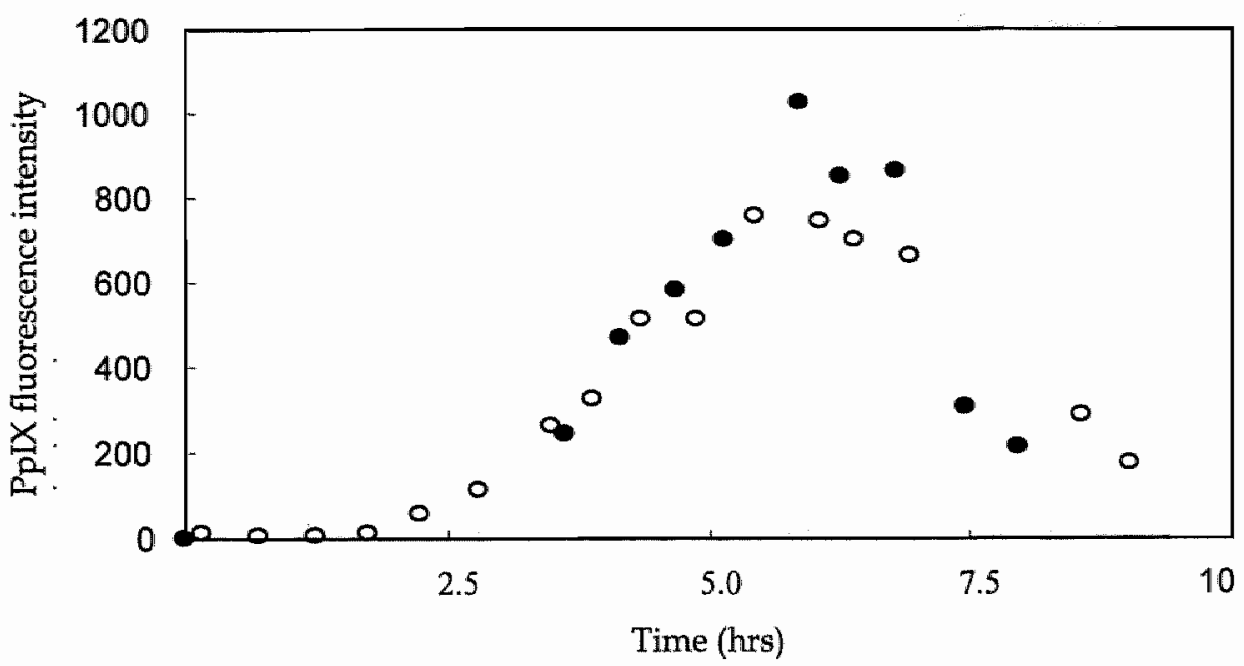

Figure 8.5

The PpIX fluorescence intensity measured at $|0|$ a distant test site and at (-) a point immediately adjacent to a test site that had received an intracutaneous injection with ALA.

Measurement of fluorescence started immediately after the injection. There was no significant difference between the maximum intensity and kinetics of $P p I X$ fluorescence between local and distant sites, indicating that (ALA and) $P p L X$ is not transported cutaneously.

\section{COMMENT}

Topical ALA-PDT can be used successfully for treatment of superficial cutaneous malignancies, but there are three possible reasons for the less favorable results of PDT for nodular tumors. The limiting factors might be insufficient penetration of the photosensitizer, insufficient penetration of the light or oxygen depletion. Theoretically, light with a wavelength of $630 \mathrm{~nm}$ can penetrate into the skin up to at least $3 \mathrm{~mm}$, although a part of the lightbeam is lost due to scattering and reflection (Svaasand,1996). Although PDT has been used for many years, the literature does not provide us with scientifically obtained data about required concentrations of photosensitizers, depth of PDT induced damage and effective irradiation schemes. The ultimate aim of this study was to find a basis for optimal fluence-irradiance schemes after intracutaneously injected ALA for PDT of nodular tumours in 
humans. The influence of the tissue oxygen concentration has not been investigated in this study.

As expected, the initial rate of intracellular PpIX accumulation after intracutaneous injection of ALA is significantly greater than that observed following topical application in the same model (de Blois, submitted). Even for time points up to 8 hours after the administration of ALA the intensity of PpIX fluorescence was significantly greater than that observed after topical application of $20 \%$ ALA. Because we were particularly interested in the kinetics of the PpIX fluorescence, we did not investigate directly the localization of PpIX by fluorescence-microscopy. Subsequently the PDT induced damage was also significantly greater and deeper after injection of ALA compared to topical application for equal irradiation schemes. Harth et al. (1998) also tried to enlarge the effect of topical PDT by adding penetration enhancers to the ALA in cream, but they failed to compare the results in the same study with ALA cream without penetration enhancers. In vivo measured fluorescence levels are correlated to the amount of PpIX concentrations in the tissue. Generally, it is assumed that the final PDT induced damage is also correlated to the fluorescence level of PpIX, but this has not been proven so far. Other factors like tissue temperature and oxygen concentration may also play an important role in this process. Orenstein et al. (1999) tried to enhance the damage after PDT by using infrared-induced tissue hyperthermia. Liu (1997) even described tumour vessel damage resulting from laser induced hyperthermia alone without the combination with PDT. Histologic changes in the control site treated with irradiation only were not abserved (Table 8.1). Juzenas (1999) on the other hand, demonstrated that the conversion of ALA into PpIX is a strongly temperature dependent process, which is even inhibited at temperatures below $12^{\circ} \mathrm{C}$. For these reasons, the temperature of the skin and the rectum temperature were constantly measured, to keep these as constant as possible, avoiding interference with the normal physiological processes in the skin.

Previously it was demonstrated that the effectiveness of ALA-PDT can be significantly enhanced by using a treatment scheme that utilises a twofold irradiation scheme where two light fractions were delivered separated by a dark period longer than 1 hour. In a transplanted rat tumour model delivering $200 \mathrm{~J} \mathrm{~cm}^{-2}$ in two fractions $\left(100+100 \mathrm{Jcm}^{-2} ; 1\right.$ and $2.5 \mathrm{~h}$ after $100 \mathrm{mg} \mathrm{kg}^{-1}$ ALA i.v.) resulted in a significant increase in tumour growth delay following PDT over that observed when $200 \mathrm{~J} \mathrm{~cm}^{-2}$ was delivered in one 
single fraction (de Bruijn,1999). Van der Veen also reported a $2.3-4.3$ times higher visual skin damage following PDT in hairless mouse skin, when $200 \mathrm{~J}$ $\mathrm{cm}^{-2}$ is delivered in two fractions $\left(100+100 \mathrm{~J} \mathrm{~cm}^{-2}\right.$; interval $\left.6 \mathrm{~h}\right)$ over that observed when $200 \mathrm{~J} \mathrm{~cm}^{-2}$ was delivered in a single fraction (van der Veen, 1999). In each of these two studies the timing of the second light fraction was chosen in order to maximise the PpIX fluorescence intensity, and thereby hopefully maximise the PDT damage. This was determined from the time at which PpIX fluorescence reached a maximum in the absence of irradiation. Subsequently, it was shown that the synthesis of PpIX after irradiation is rather more complex. In particular, Robinson (1999) reported that PpIX synthesis after PDT in a transplanted rat tumour model is a function of the fluence delivered during PDT; delivering a low fluence results in the reappearance of PpIX fluorescence after irradiation whereas delivering a high fluence largely inhibits the re-synthesis of PpIX. The results presented in our study confirm these findings. Following 3 and $6 \mathrm{~J} \mathrm{~cm}^{-2}$, the intensity of PpIX fluorescence increases after photobleaching during irradiation and has returned to its pre-treatment intensity $2 \mathrm{~h}$ after the end of the irradiation. With increasing fluence the re-synthesis of PpIX is increasingly inhibited and for fluences above $15 \mathrm{~J} \mathrm{~cm}^{-2}$ PpIX fluorescence intensity does not increase significantly for any of the time points investigated.

Although expected, there was no significant effect on PDT-induced skin damage of different PpIX fluorescence intensities at the time of the second irradiation. Delivering a low fluence in the first fraction $\left(3+27 \mathrm{~J} \mathrm{~cm}^{-2}\right.$ and $6+24 \mathrm{~J} \mathrm{~cm}^{-2}$ ) results in a significantly higher PpIX fluorescence intensity at the time of the second light fraction, than when $15 \mathrm{~J} \mathrm{~cm}^{-2}$ is delivered in the first fraction. Similarly, it was unexpected that $3+27 \mathrm{~J} \mathrm{~cm}^{-2}$ and $6+24 \mathrm{~J} \mathrm{~cm}^{-2}$ both did not prove to cause significantly more dlamage than when $30 \mathrm{~J} \mathrm{~cm}^{-2}$ was delivered in a single fraction, despite the fact that a significantly greater total amount of PpIX is photobleached when the 2-fold irradiation scheme is used. The most effective 2 -fold illumination scheme investigated in this study proved to be when $30 \mathrm{~J} \mathrm{~cm}^{-2}$ was delivered in a 2fold irradiation scheme $\left(15+15 \mathrm{~J} \mathrm{~cm}^{-2}\right.$; interval $\left.2 \mathrm{~h}\right)$.

The percentage of PpIX photobleaching following the second light fraction is relatively less than that following the first irradiation, even when the same fluence is delivered in each fraction. Also the difference in photobleaching of PpIX between the first and second fraction increases with increasing fluence delivered in the first fraction. It has been demonstrated 
previously that the rate of PpIX photobleaching depends on the local oxygen concentration (Robinson, 1999). Hypothetically, the reduction in photobleaching following the second light fraction might be a result of a decrease in the availability of oxygen caused by vascular damage caused in the first irradiation. This was not further investigated.

PDT-induced damage does not increase significantly when the irradiance is reduced from 100 to $50 \mathrm{~mW} \mathrm{~cm}^{-2}$. This is contrary to the results of other studies, which have demonstrated an increase in the PDT damage with decreasing fluence rate (Robinson,1998 and 1999). They reported that reducing the fluence rate from 150 to $50 \mathrm{~mW} \mathrm{~cm} \mathrm{~cm}^{-2}$ resulted in a significant increase in the visual skin damage score following PDT of normal and UVB induced tumours in hairless mouse skin. This effect was even greater when the irradiance was reduced to $5 \mathrm{~mW} \mathrm{~cm}^{-2}$. Van der Veen (1999) also demonstrated an increase in PDT damage of normal hairless mouse skin when the irradiance was reduced from 100 to $50 \mathrm{~mW} \mathrm{~cm}$ chly $^{-2}$ (onlynificant for $t=10$ but not for $t=4$ ), however these authors did not report a corresponding increase in damage in UVB treated skin. The authors suggested that the absence of a fluence rate effect in this case may be due to the high concentration of PpIX in UVB treated skin that causes oxygen depletion even at $50 \mathrm{~mW} \mathrm{~cm} \mathrm{~cm}^{-2}$. This may also explain the absence of a fluence rate effect in our model where we have shown significantly higher fluorescence intensities following intracutaneous administration of ALA than those after topical application. These data might suggest that a further reduction in fluence rate is necessary to maximise PDT induced damage (Robinson, 1999).

\section{CONCLUSION}

Summarizing, in vivo ALA-PDT studies on normal porcine skin were performed, investigating both the fluorescence kinetics as a reflexion of $\mathrm{PpIX}$ concentration in the tissue, and the final PDT-induced tissue damage for different irradiation schemes after intracutaneous injection of ALA compared with topically applied ALA. This study clearly demonstrates that intracutaneous administration of ALA leads to the formation of PpIX more rapidly and in higher concentrations compared with topical application of ALA. Besides that, the final PDT-induced skin damage is significantly greater if the total light dose for irradiation is given in two equal fractions, separated by a dark interval of 2 hours. There is no obvious reason to expect that the 
PpIX fluorescence kinetics and PDT-induced skin damage in human skin will differ largely from that in porcine skin. So, the results of this study justify further investigation of the effectiveness of PDT for nodular tumours in humans with intracutaneous administration of ALA, followed by the irradiation scheme as mentioned above after 3.5 hours, using lower irradiances than those that have been generally used (Cairnduff, 1994; Wennberg, 1996; Hurlimann, 1998). The time involved for this total PDT treatment will be six hours, making it practically possible to perform the treatment within one working day.

\section{Acknowledgements}

The authors would like to thank Erbe Nederland B.V. for providing the light source used in this study, and the members of the Central Animal Institute, Maastricht University for their assistance with preparation, anesthesia and care of the animals used in this study.

DJ Robinson and HS de Bruijn are supported by the Dutch Cancer Society (project DDHK-98-1686). 


\section{Chapter Nine}

GENERAL DISCUSSION and

RECOMMENDATIONS 
Basal cell carcinoma $(\mathrm{BCC})$ has become a serious health problem and incidence rates of this tumour are still increasing worldwide. In the Netherlands the current estimated annual incidence at the beginning of the new millennium will exceed 30.000 cases. In some parts of Australia and the United States these rates are much higher. The two main reasons for the increase in incidence are the ageing of the population and the changes in sunbathing behaviour starting in the 1970's, including the abundant use of artificial sunbeds. Especially persons with a fair skin type and poor tanning ability, who are frequently exposed to high doses of ultraviolet (UV) radiation are at risk to develop BCCs. Additional risk factors are impaired immunity, male sex and some genotypical factors as in xeroderma pigmentosum and nevoid basal cell syndrome. These and more detailed epidemiologic data are summarized in the first chapter of this thesis.

For the future, it is to be expected that more and more people will develop multiple BCCs at younger age on visible parts of the body, especially in the head/neck area. Cure, of course, remains the primary goal of treatment of these tumours. However, besides this curative aspect, the final cosmetic and functional result of a typical treatment will become more and more important. This thesis further deals with the different treatment modalities for BCC: principles and practice, advantages/disadvantages, indications and of course the effectiveness, both from a curative and from a cosmetic point of view, are described. The conclusions and recommendations in this thesis are based on data obtained from literature search and clinical research.

\section{REMARKABLE FINDINGS}

\section{Literature Search / Evidence Based Medicine}

Systematically searching the literature for studies reporting on treatment of $\mathrm{BCC}$, we discovered the amazing fact that, despite the high incidence rates of $\mathrm{BCC}$, in literature there is an enormous lack of clinical investigations of the effectiveness (5-year recurrence rates) of the different treatment modalities for $\mathrm{BCC}$, really performed as should be according to the principles of evidence based research in cancer: large, prospective (comparative) studies, for primary and recurrent BCCs separately, with long term follow-up for at 


\section{Chapter Nine}

least 5 years. Most studies in literature, however are retrospective analyses, with a follow-up shorter than 5 years, reporting on treatment of both primary and recurrent BCCs or on non-melanoma-skin-cancer in general. The systematic literature search, as described in detail in chapter 3 , shows that surgical intervention, and particularly Mohs" micrographic surgery, is the treatment modality for $\mathrm{BCC}$ with the lowest 5-year recurrence rate. Surgical intervention remains the "golden standard" therapy. It would be advisable to perform large, prospective, (randomized, comparative) studies on primary $\mathrm{BCCs}$, according to strict protocols, with long term follow-up (at least 5 years for each tumour treated) for one or more treatment modalities in future, to obtain reliable information about the real 5 -year recurrence rates of these therapies.

\section{Cosmetic Results}

Secondly, it is remarkable that there is only little attention for the difference in cosmetic and functional results of the different treatment modalities. Sometimes the assessment of the cosmetic results by the patients is mentioned in passing, but studies objectively comparing the cosmetic results of two treatment modalities are hard to find. How can this be possible, since we are living in a luxurious world today, where every accumulation of fat, each furrow or wrinkle has to be removed? A plausible explanation for this phenomenon might be the fact that in the past, BCCs almost always appeared in elderly persons, for whom the cosmetic results perhaps were less important. But time has changed now.

According to the results of chapter 4 , the cosmetic results after cryosurgery and surgical excision (the two treatment modalities which are performed most frequently) for primary uncomplicated BCCs in the face in an average population, were obviously in favor of the surgical excision. In this new millennium however, for the reasons as mentioned already before, the treatment of BCCs should be performed avoiding any risk for cosmetically inferior results in everyone treated for this tumour.

\section{Biopsies and BCC}

The third and final striking aspect appears from the inquiry among the dermatologists in the Netherlands, as described in chapter 5 . In view the fact, that the public health care in our country operates on a high level (both diagnostically and therapeutically), the percentage of biopsies taken from 
lesions suspect for $\mathrm{BCC}$ to confirm the clinical diagnosis is relatively low. This implies, for example, that treating a $\mathrm{BCC}$ without a biopsy by cryosurgery, means that we are treating a lesion without having an absolutely clear diagnosis, without being informed about the growth pattern and without the possibility of histological evaluation after therapy; summarizing, with all risks of treating this lesion inadequately, leading to more difficulties in the future.

\section{PHOTODYNAMTC THERAPY}

In cases of multiple BCCs, both in the head/neck area and on the rest of the body, the number of tumours that can be surgically excised in a certain area will be limited, depending on the amount of surrounding skin that can be used for reconstruction of the defect. This is a serious reason why the demand for non-invasive, adequate and effective therapies for treatment of these tumours is increasing. Cryosurgery, and also radiotherapy and curettage/electrodesiccation, are non-surgical (i.e. non-sharp) procedures, but they are also non-selective which means that besides tumour cells a relatively large amount of surrounding healthy tissue will be destroyed too. The final cosmetic result after several years often becomes of inferior quality. Photodynamic therapy is a promising "re-newed" option for such noninvasive, selective treatment of BCCs resulting in excellent cosmetic results. For the treatment of skin tumours and precancerous lesions photodynamic therapy with topical administration of 5-ALA is of increasing interest. In this way basal cell carcinomas have been treated predominantly in small, notstandardized clinical studies with limited follow-up. Short term complete response rates reported in literature are high (90-100\%) for BCCs of the superficial subtype, but for the nodular type they are less favorable varying from 10 to $64 \%$.

The reasons for these disappointing results may be insufficient penetration of either the light or the topically applied photosensitizer (5-ALA in cream), or maybe a deficiency in oxygenation of the treated tissue. Theore tically, a lightbeam of $630 \mathrm{~nm}$ penetrates into the skin untill the deep dermal layer, which should be deep enough to reach the bottom of superficial and nodular BCCs. Oxygen, however, is essential for the process of PDT. Nodular BCCs may also present with an ulcerated, necrotic centre (ulcus rodens) lacking vascularization and oxygenation, thus possibly resulting in less PDT- 
induced damage. On the other hand, the problem of oxygen depletion in well vascularized tumours could be diminished by performing a pulsed or fractionated illumination, thus creating a possibility for re-oxygenation of the tissue during the dark intervals.

Two procedures for the process of PDT can be used to get around the difficulty of insufficient amounts of the photosensitizer in the deeper parts of nodular tumours. Partial debulking of the upper part of the tumour, performed before topical ALA-PDT, will reduce the total tumour mass, therefore facilitating the penetration of 5-ALA into the bottom of the tumour. This modified application of topical PDT for nodular tumours obviously resulted in a higher percentage of histologically confirmed complete responses, as shown in chapter 6 . The partial debulking did not influence the final cosmetic result in a negative way.

The second method to obtain deeper localized amounts of photosensitizer is by intralesional injection of 5-ALA. For this purpose, a 5-ALA containing solution was developed, that could be injected safely, not causing too much discomfort for the patient. In vivo dose-response studies on animals revealed that the concentrations of 5-ALA in the injection solution, necessary to cause the same effect as with the cream, were only $10 \%$ of the concentration used in the cream (chapter 7). After injection of 5-ALA, a sufficient amount of the photosensitizer PpIX was also formed in a shorter time interval compared to the situation when the cream was used. Further research demonstrated that the PDT-induced tissue damage also could be enhanced (both more deeply localized and more severe damage) performing fractionated illuminations with a dark interval of two hours instead of single illuminations (chapter 8). From a practical point of view, these observations mean that the time between administration of 5-ALA and illumination can be shortened and that even a subsequent second illumination can be performed on the same day within a time period of six hours, which is practically acceptable for the patient.

The results of the preclinical studies on intracutaneous injection of 5ALA and the influence of different irradiation schemes justify further investigation of these aspects in patients with primary nodular BCCs. Other, more agressive histological subtypes of $\mathrm{BCC}$, should not be treated with PDT as long as this treatment modality is investigational. However, large, prospective studies comparing the effectiveness of PDT to other current therapies, like surgical excision, are necessary before PDT can be used as a 
standard therapy for BCCs.

\section{RECOMMENDATIONS}

Diagnostic procedures (flow-chart I, p 139)

Before any treatment is performed at all, it is recommended to take a representative, deep, full-thickness skin biopsy of 3 or $4 \mathrm{~mm}$ in diameter from the lesion clinically suspicious for $\mathrm{BCC}$ for histopathologic examination, to have both the clinical diagnosis confirmed and to obtain reliable information about the histologic classification of the tumour. Most BCCs can be histologically classified as the non-agressive solid/nodular (45-60\%) and superficial (15-35\%) types, or as the agressive (4-17\%) and more infiltrative growing morpheic (sclerosing), micro-nodular or adenocystic subtypes. In cases where histologic examination does not confirm the clinical diagnosis (i.e. is negative), a revision of the microscopic examination is indicated and if the results are still negative despite the high clinical suspicion, a new fullthickness biopsy should be taken. The one and only exception on this procedure is the patient with multiple BCCs of the superficial subtype; treatment may be performed without taking a biopsy from each tumour separately.

The question may arise whether stitches should be used to close the biopsy defect or not. There are three good reasons for leaving this small defect open. From an oncologic point of view, any additional or needless sharp manipulation of the tumour might be a risk for contaminating and seeding the oncologic woundbed. In the second place, usually the consistence of the tumour is so weak that a suture will easily tear out. Finally, the biopsy-procedure is nearly almost followed by the definitive treatment of the tumour, so the small biopsy defect will be entirely removed afterwards.

\section{Treatment of BCC (flow-chart II, p 139)}

Coming to the end of this thesis, this is the moment to recommend which strategy one should follow in making the decision "how to treat $\mathrm{BCC}$ " or, maybe even more important, "how NOT to treat this tumour". The answer to these questions is mainly determined by the histologic classification of the BCC. Additional factors in this determination are the size/volume and localization of the tumour, the combination of general health and age of the patient and also the question whether the tumour is a primary or recurrent one. In general, BCCs larger than $2 \mathrm{~cm}$ in diameter, those localized in the 
central part of the face (the so called H-zone) and recurrent BCCs tend to grow in a more agressive way.

Practically, the dermatologist can make a decision for the treatment of $\mathrm{BCC}$ out of the following three standard therapies: conventional surgical excision, Mohs' micographic surgery and cryosurgery. For all BCCs, and especially for those more or less agressive tumours as mentioned previously, surgical treatment is the preferential therapy, because this is the most precise and safest treatment modality with possibilities for histologic evaluation on removal of the tumour afterwards. At this point, it should be noted that routine histopathological evaluation of the resection margins following conventional surgical excision, examining several vertical sections (breadloaf technique), has limitations concerning the reliability of the technique. But for practice, it will not be necessary to examine each $\mathrm{BCC}$ histopathologically in a three dimensional way. However, for the agressive BCCs, precise histopathologic examination is absolutely required, and for these tumours, Mohs' micrographic surgery is an excellent treatment. On the other hand, treating all BCCs with Mohs' micrographic surgery will certainiy also lead to overtreatment. Therefore, the smaller $(<2 \mathrm{~cm})$, uncomplicated, primary $\mathrm{BCCs}$ can be treated effectively by conventional surgical excision, whereas the larger (>2 cm) and more agressive primary $\mathrm{BCCs}$ and the recurrent tumours are indications for Mohs' micrographic surgery.

Cryosurgery is the first alternative treatment in cases where contraindications to surgery exist, on the condition that it is performed in a standardized way. There are two situations in which cryosurgery is even recommended as the first-line therapy. The first indication are clinically well delineated, primary, superficial and nodular growing BCCs smaller than 2 $\mathrm{cm}$ and localized on the eyelids and helix, because cartilage (tarsus and helix) and the ductus lacrimalis (which need to be preserved) are resistant to freezing. The second indication is the patient with multiple small superficial BCCs on the trunk. Two absolute contra-indications for cryosurgery are existing intolerance to cold and the morpheic or sclerosing histologic subtype of BCC.

All other current treatment modalities for $\mathrm{BCC}$ should be used only in situations where serious contra-indications to surgical intervention or cryosurgery exist. The reasons for this are the higher recurrence rates and the inferior (long term) cosmetic results of these treatment modalities and the aggravation for the patients in case of radiotherapy (frequent visits). 
The effectiveness of the investigational therapies, like topical interferoninducers and photodynamic therapy, has to be evaluated and compared to other standard therapies in large prospective, randomized studies, before these treatment modalities can be added to the current therapies.

\section{Follow-up}

Patients with $\mathrm{BCC}$ have a serious increased risk for developing more $\mathrm{BCC}$ during their lives. On the other hand, recurrences of previously treated BCCs develop most frequently between 3 and 10 years after treatment. For these two reasons, it is advisable to maintain a long term (at least 5 years) follow-up with visits at regular times. The guidelines for follow-up, as proposed by the Dutch Society for Dermatology and Venereology (NVDVGuidelines Technical Committee; follow-up every 3 months for the first year, twice in the second year, and thereafter once a year including "total-bodyskin-check") should be followed for every patient with a $\mathrm{BCC}$, to detect a new or recurrent $\mathrm{BCC}$ in an early phase, which makes them easier to treat.

\section{Prevention}

The increase in the incidence of BCC is critical. The most important way to reduce the incidence of $\mathrm{BCC}$ is prevention. Primary cancer prevention has to be focused on convincing people that excessive exposure to ultraviolet radiation (either from natural sunlight or artificial sunbeds) is unhealthy, especially when this occurs during early childhood and adolescence. Therefore, parents should take effort to protect their children against sunlight, because their skin is very susceptible to repeated UV-induced DNA-damage, which becomes clinically evident many years later (multiple-step mechanism in the development of $\mathrm{BCC}$ ). Furthermore, it should be wise for everyone to avoid excessive exposure to sunlight between 10.00 am and $16.00 \mathrm{pm}$, to use effective (SPF > 15 against both UVA and UVB radiation) sun-protection creams several times a day, and to wear thightly woven, protective clothing, sunglasses and hats to protect the face against sunlight.

Another, different way of prevention is secondary prevention. However, secondary prevention (or volunteer screening) in cancer is generally aimed at early detection of tumours, with the intention to treat them at an early stage, thus significantly reducing the mortality and morbidity of this disease. Although $\mathrm{BCC}$ is an important health problem, the benefit of screening for this tumour is doubtful. $\mathrm{BCC}$ has a low mortality rate. The problems which 
may result from these tumours are totally different from those of other malignant, metastatic diseases and need their own typical approach. On the other hand, people should be encouraged to self-awareness on persistent, non-healing skin lesions and seeking medical help in such cases, because treatment of $\mathrm{BCC}$ in an early stage may reduce the risk for large, cosmetically disturbing scars following treatment.

Summarizing, the money which is available for prevention of $\mathrm{BCC}$ should be better spent on educational programmes rather than on screenings campaigns. 


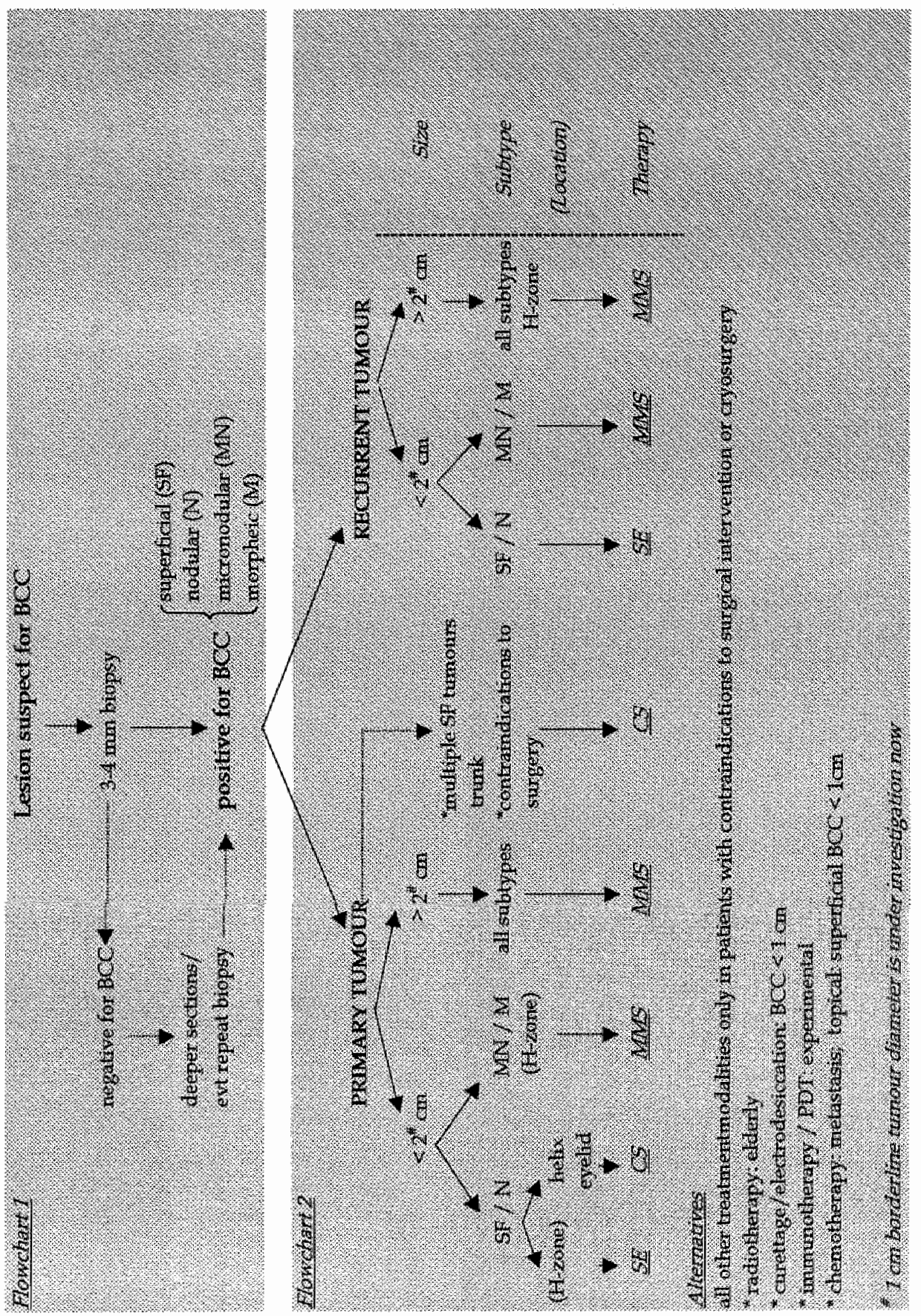




\section{SAMENVATTING}


Het carcinoma basocellulare (basaalcelcarcinoom, BCC) zoals wij dat kennen, dateert al uit 2000 jaar v.Chr., hetgeen gebleken is tijdens opgravingen in Egypte waarbij de skeletten van mummies reeds kenmerken vertoonden die wij nu kennen als passend bij het basaal cel naevus syndroom. Door de eeuwen heen heeft deze tumor steeds meer bekendheid gekregen. Het $\mathrm{BCC}$ is nu wereldwijd de meest voorkomende huidmaligniteit, die hoofdzakelijk ontstaat onder invloed van UV (zon)licht, waarbij de vergrijzing van de bevolking en het veranderde zongedrag van de laatste decennia een belangrijke rol hebben gespeeld -en nog steeds spelen- in de haast dramatische stijging van de incidentie cijfers van deze tumor.

In dit proefschrift worden de verschillende gangbare behandelingsmodaliteiten voor het $\mathrm{BCC}$, alsmede een nieuwe experimentele therapie nader geëvalueerd op basis van hun effectiviteit en cosmetisch eindresultaat, om zodoende uiteindelijk aanbevelingen te kunnen doen voor diagnostiek, optimale behandeling, follow-up en preventie van het BCC.

\section{HOOFDSTUK EEN}

In het eerste hoofdstuk worden de epidemiologische gegevens betreffende registratie, incidentie, etiologie/risicofactoren, morbiditeit/mortaliteit en preventieve maatregelen omtrent het BCC nader beschreven voor Nederland, noord- en west-Europa en de rest van de wereld. Het blijkt dat er wereldwijd, behoudens voor het melanoom, geen nauwkeurige registratie bestaat voor huidkanker, en dus ook niet voor het BCC. De incidentie van het BCC in Nederland werd in 1994 geraamd op 18.000 nieuwe tumoren per jaar. Op het einde van het vorige millennium leek het (geschatte) aantal reeds rond de 30.000 te liggen, hetgeen duidelijk meer was dan men op grond van eerdere getallen had verwacht. Ook in de rest van Europa, de Verenigde Staten, midden-Amerika en Australië wordt een soortgelijke incidentiestijging gezien, al liggen de absolute getallen in de laatste drie gebieden veel hoger dan in Nederland. Dit heeft waarschijnlijk alles te maken met de excessievere blootstelling aan zonlicht in deze gebieden, hetgeen de belangrijkste risicofactor is voor het ontwikkelen van BCC's, vooral wanneer dit geschiedt tijdens de kinderjaren en de vroege adolescentie. Eventuele andere 
exogene risicofactoren kunnen zijn excessieve/chronische blootstelling aar ioniserende straling of chemische carcinogenen of psoralen (PUVA therapie) Enkele patiëntgebonden risicofactoren daarentegen zijn een licht huidtype geringe neiging tot bruin worden bij blootstelling aan zonlicht, het mannelijl geslacht, en verdere een aantal genotypische factoren die gepaard gaan me een stoornis in het herstel van door zonlicht geïnduceerde DNA schade, zoal xeroderma pigmentosum en het basaal-cel-naevus-syndroom. Ook een min der goed functionerend immuunsysteem, een voorgeschiedenis of familie anamnese met $\mathrm{BCC}$, alsmede het hebben van een predisponerende derma tose (nevus sebaceus, Pinkus' tumor) kunnen de kans op een BCC vergroten Van alle histologische sub-types blijkt het nodulaire/solide type het mees voor te komen (45-60\%), gevolgd door het superficiele type (15-35\%). Do meer agressieve, en vaker infiltratief groeiende types zijn duidelijk in de minderheid (4-17\%). Ongelukkigerwijze, maar wel verklaarbaar, ontstaan d meeste BCC's op de aan zonlicht blootgestelde delen zoals het gelaat en de rug. Het $\mathrm{BCC}$ is een tumor die zelden metastaseert en derhalve ook een zee: lage mortaliteit kent, maar bij onvolledige of te late behandeling kunnen me name de agressieve types tot mutilerende littekens en derhalve tot grot morbiditeit leiden.

Vanuit preventief oogpunt is met name de primaire preventie van be lang om de incidentie stijging een halt toe te roepen. Naast het onder de aan dacht brengen van het feit dat een gebruinde huid wel populair moge zijn maar zeker niet gezond is, moeten patiënten gewezen worden op het posi tieve aspect van zelf-onderzoek en op het vermijden van (exogene risicofactoren. Het nut van secundaire preventie in de vorm van (vrijwillige deelname aan) screeningscampagnes voor het BCC is twijfelachtig, omda het $\mathrm{BCC}$ geen invloed heeft op de uiteindelijke (over)levingsduur van de patiënt na het stellen van deze diagnose.

\section{HOOFDSTUK TWEE}

Op grond van de 5-jaars recidief percentages uit de literatuurstudie er het cosmetisch resultaat op de lange termijn, lijkt het gerechtvaardigd on voor de behandeling van het BCC een keuze te maken uit een drieta standaard therapie modaliteiten, te weten Mohs' micrographische chirurgie chirurgische excisie en cryochirurgie. Van deze behandelingsmethoder worden in dit hoofdstuk de historie, het werkingsprincipe en de praktische toepassing nader uiteengezet. Omdat het laatste deel van dit proefschrif 
over de -nog steeds experimentele- photodynamsiche therapie handelt, wordt ook van deze behandelingsvorm de ontwikkeling, het werkingsmechanisme en de praktische uitvoering beschreven.

\section{HOOFDSTUK DRIE}

In dit hoofdstuk wordt de systematische analyse beschreven van de in de wereldliteratuur gepubliceerde studies over de resultaten (recidief percentages) van verschillende therapie modaliteiten die voor behandeling van het BCC worden toegepast. Hiervoor werden alle, tussen 1970 en 1997 gepubliceerde, engels-, frans-, duits- en nederlandstalige, alsmede de spaanse en italiaanse studies geëvalueerd, die rapporteerden over recidief percentages van $\mathrm{BCC}$ na de volgende behandelingen: Mohs' micrographische chirurgie, conventionele chirurgische excisie, cryochirurgie, curettage/electrodesiccatie, radiotherapie, immunotherapie met interferon en fluorouracil en photodynamische therapie. Van alle studies die aan bovengenoemde criteria voldeden werd op kritische wijze de opzet en uitvoering gewaardeerd. Alleen prospectief uitgevoerde studies, bij minimaal 50 primaire (onbehandelde) BCC's die minimaal 5 jaar vervolgd waren, werden gebruikt in een poging om het aanvankelijke doel van deze literatuurstudie te realiseren: het opstellen van richtlijnen voor de behandeling van het $\mathrm{BCC}$ op grond van gewogen gemiddelde 5-jaars recidief percentages van de verschillende therapie modaliteiten.

Van de 298 studies die geselecteerd werden uit de verschillende electronische databases (Medline Standard en Advanced, Embase, Cancerlit), dermatologische handboeken en naslagwerken, reviews, bestaande richtlijnen en referenties van de gevonden studies, voldeden er slechts 18 aan de gestelde criteria. Bij de analyse werd duidelijk dat er absoluut geen onderlinge uniformiteit in de wijze van rapportage van de gegevens bestond tussen deze studies. Er werden ofwel "ruwe", dan wel "exact" berekende 5-jaars recidief percentage vermeld. Slechts in enkele studies werd het cumulatieve 5 -jaars recidief percentage gegeven, terwijl dit in sommige andere studies wel uit de gegevens berekend kon worden. De recidief percentages die in de betreffende studies waren vermeld konden derhalve geenszins met elkaar vergeleken worden, om op grond daarvan evidence-based richtlijnen voor de behandeling van BCCs op te stellen. Het bleek dat tumoren die met Mohs" micrographische chirurgie waren behandeld na 5 jaar het laagste recidief percentage vertoonden $(0,8-1,1 \%)$, successievelijk gevolgd door de conven- 
tionele excisie, cryochirurgie, radiotherapie en curettage/electrodesiccatie.

Praktisch gezien betekent dit zeker niet dat alle BCC's nu met Mohs" micrographische chirurgie moeten worden behandeld, hetgeen tot absolute overbehandeling zou leiden. Deze speciale techniek zou vooral toegepast moeten worden bij grotere tumoren met een agressieve (histologische) groeiwijze, gelokaliseerd in de $\mathrm{H}$-zone van het gelaat, en bij recidief tumoren. De overige BCC's kunnen effectief met de conventionele excisie behandeld worden. De andere behandelingsmodaliteiten zouden beter gereserveerd kunnen worden voor situaties waarbij er contra-indicaties voor chirurgische interventie bestaan.

\section{HOOFDSTUK VIER}

Hoewel de cryochirurgie voor de behandeling van het $\mathrm{BCC}$ nog op grote schaal wordt toegepast, is het cosmetisch resultaat van deze therapie op de langere termijn niet altijd even fraai, waarbij met name de localisatie en de natuurlijke huidskleur/huidtype mede het eindresultaat zullen bepalen. In dit hoofdstuk worden de cosmetische eindresultaten een jaar na cryochirurgie en chirurgische excisie van primaire nodulaire en superficiële BCC's in de hoofd/hals regio met elkaar vergeleken aan de hand van gestandaardiseerde dia's (overzicht en detail) die direct voor en 1 jaar na de behandeling zijn genomen. De beoordeling van de resultaten ("goed, matig of slecht") geschiedde zowel door de patiënt zelf, als door een onafhankelijk panel, bestaande uit een mannelijke dermatoloog, een vrouwelijke dermatologe/Mohs' chirurge, een plastisch chirurg, een dermatologisch verpleegkundige en een schoonheidsspecialiste, die allen niet eerder in de studie geparticipeerd hadden en die ook niet op de hoogte waren welke therapie bij welke patiënt was toegepast.

Zowel door de patiënt als door de panel-leden werden de resultaten na cryochirurgie als inferieur ten opzichte van de resultaten na excisie beoordeeld. Alleen voor de schoonheidsspecialiste was deze voorkeur voor excisie niet significant. De mate van overeenkomst in de beoordeling (bepaald als de kappa-waarde) tussen de panel-leden onderling was relatief hoog. De patiënten daarentegen waardeerden de resultaten in het algemeen duidelijk beter dan de panel-leden. Voor de mannelijke dermatoloog als enige, was de localisatie van de ingreep van invloed op zijn beoordeling; de resultaten na excisie in de regio wang, periauriculair en nek werden als kwalitatief minder goed beoordeeld dan in de rest van het gelaat (neus, kin, peri-oculair en 
voorhoofd/slaap].

Deze gerandomiseerde, prospectief vergelijkende studie toont aan dat het voor de dermatoloog van de toekomst belangrijk is om over ruime ervaring in chirugische ingrepen te beschikken, om zodoende de patiënt een optimale behandeling te bieden, zowel vanuit curatief als vanuit cosmetisch/ functioneel opzicht.

\section{HOOFDSTUK VIJF}

Om een indruk te krijgen van de wijze waarop het BCC door de nederlandse dermatologen gediagnostiseerd en behandeld wordt, werd een anonieme, schriftelijke enquête gehouden onder deze beroepsgroep. Aan alle 293, in Nederland practiserende dermatologen werd in 1996 een lijst toegestuurd met 15 vragen betreffende deze twee aspecten. In totaliteit kwamen 18 lijsten te vervallen, wegens beëindiging van de praktijk of omdat er 1 lijst per vakgroep was ingevuld. De uiteindelijke respons op deze enquête bedroeg $76 \%$ (208 van de 275), waardoor de resultaten als representatief mogen worden beschouwd voor de gemiddelde dermatologische praktijk in Nederland. De diagnose BCC werd doorgaans, doch niet altijd, op grond van histopathologisch onderzoek gesteld $(71 \%$ van de respondenten; $84 \%$ in geval van recidief tumorenj. Voor alle subtypen $\mathrm{BCC}$ bleek de chirurgische excisie de voorkeursbehandeling. Als tweede keuze gold de cryochirurgie of de curettage/electrodesiccatie. Röntgencontacttherapie werd vrijwel niet meer toegepast. Nieuwe behandelingen zoals fotodynamische therapie en immunotherapie werden slechts sporadisch op experimentele basis toegepast. De meerderheid van de BCC's werd door de dermatologen zelf behandeld; een enkele keer vond doorverwijzing plaats naar de plastisch chirurg, met name bij de grotere tumoren. De meeste dermatologen beschouwden recidief tumoren als een groter probleem dan primaire tumoren. Door adviezen te geven ten aanzien van risicofactoren (zonlicht) probeerden zij de ontwikkeling van nieuwe BCC's te beperken.

Concluderend kon gesteld worden dat er nog onvoldoende gebruik werd gemaakt van de diagnostische biopsie, zeker bij recidief tumoren, om een optimale therapiekeuze te kunnen maken bij de behandeling van het BCC.

\section{HOOFDSTUK ZES}

Vanwege de forse stijging van het aantal personen met multipele BCC's 
in het gelaat, maar ook op de romp, bestaat er een toenemende behoefte aan niet-inwasieve therapie modaliteiten die bij voorkeur ook nog selectief op het tumorweefsel werken; dit in tegenstelling tot de niet-scherpe, maar ook nietselectieve cryochirurgie en radiotherapie, waarbij naast tumorweefsel tevens een hoeveelheid gezond weefsel wordt gedestrueerd, hetgeen tot cosmetisch minder fraaie resultaten kan leiden. Photodynamische therapie (PDT) is een dergelijke niet-invasieve, selectieve behandelingsmodaliteit, die echter nog in een fase van onderzoek verkeert. Het werkingsprincipe berust op een tumorgelokaliseerde fotosensibele stof die geactiveerd wordt door licht van een bepaalde golflengte, waarbij o.a. cytotoxische producten ontstaan die uiteindelijk celdood in de tumor induceren. Met name de oppervlakkig groeiende BCC's kunnen effectief behandeld worden met PDT, gebruikmakende van de topicaal toegepaste fotosensitizer 5-aminolevulinezuur (ALA), maar bij de dikkere nodulaire BCC's die nu juist het meest voorkomen, lijkt de penetratie van ALA of van de lichtbundel een beperkende factor te zijn.

In hoofdstuk zes wordt de effectiviteit van topicale ALA-PDT onderzocht bij een groep van 24 nodulaire BCC's, waarbij drie weken voorafgaand aan de behandeling een partiële debulking van de tumormassa werd verricht, met als doel om zodoende de penetratie van zowel de sensitizer als de lichtstraal tot in de bodem van de tumor mogelijk te maken. Op de dag van de behandeling werd $20 \%$ ALA creme op en rond het BCC aangebracht, waarna deze tumor gedurende 6 uur met lichtwerend occlusief verband werd afgedekt. Aansluitend werd de tumor eenmalig continu met $630 \mathrm{~nm}$ belicht (totale lichtdosis van $120 \mathrm{~J} \mathrm{~cm}^{-2}$ bij een intensiteit van $100 \mathrm{~mW} \mathrm{~cm}^{-2}$ ). Drie maanden na PDT werden alle "lesies" geëxcideerd en histopathologisch onderzocht op tumorresten.

Bij 2 van de 24 behandelde tumoren werden na drie maanden klinisch nog tumorresten waargenomen, hetgeen ook histopathologisch werd bevestigd. Bij de overige $22 \mathrm{BCC}$ 's waren zowel klinisch als histologisch geen tumorcellen meer te traceren, hetgeen het "volledige respons"-percentage van $92 \%$ zou kunnen betekenen. Hierbij dient echter wel opgemerkt te worden dat voor de histologische controle gebruik werd gemaakt van standaard histopathologisch onderzoek, waarbij tumorcellen soms gemist kunnen worden. De resultaten van deze studie rechtvaardigen echter toch de verdere toepassing van deze gemodificeerde toepassing van topicale PDT bij nodulaire BCC's in een grotere, prospectieve studie met langere follow-up, om het werkelijke 5-jaars recidief percentage van deze therapie te kunnen 
bepalen.

Het cosmetisch resultaat na PDT bleek bij alle behandelde tumoren excellent te zijn. Verder werden er geen bijwerkingen of complicaties waargenomen.

\section{HOOFDSTUK ZEVEN}

Een tweede manier om de sensitizer bij PDT dieper in de nodulaire tumoren te krijgen, zou de intratumorale injectie van ALA kunnen zijn. Deze techniek was echter tot dusverre niet beschreven in de literatuur. Derhalve werd eerst een stabiele, isotone ALA-oplossing met een $\mathrm{pH}$ van 5 ontwikkeld. Vervolgens werd in een diermodel, op gezonde huid, toxicologisch en dosisrespons onderzoek uitgevoerd met verschillende concentraties en volumina van de injectievloeistof, zoals beschreven in hoofdstuk 7. Hierbij werd de kinetiek van de door ALA geïnduceerde protoporfyrine IX-fluorescentie aan het oppervlak van de huid voor elke dosis gemeten en vergeleken met de waarden bij topicale toepassing van $20 \%$ ALA creme (de mate van fluorescentie kan als een indicatie gezien worden voor de hoeveelheid aanwezige sensitizer in het weefsel, en dus indirect als een maat voor het te verwachten effect van PDT). Aansluitend werd de belichting uitgevoerd en na 48 en 96 uur werd de door PDT geïnduceerde schade aan de huid zowel visueel als histologisch onderzocht voor de verschillende concentraties/volumina.

Deze in vivo studie toonde aan dat intracutane injectie van $3 \mathrm{mg}$ ALA of meer, leidt tot het sneller ontstaan van significant hogere fluorescentie waarden dan na topicale toepassing van de $20 \%$ ALA creme. Het fluorescentieniveau na intracutane toediening van ALA tot een hoeveelheid van $10 \mathrm{mg}$ bleek een dosis-afhankelijk mechanisme te zijn. Bij toediening van hogere doses werd geen verdere toename van de fluorescentie meer gezien, hetgeen zou kunnen betekenen dat de omzetting van ALA in PpIX vanwege een verzadiging van de enzymsystemen constant blijft. Het fluorescentie-niveau na topicale applicatie van $20 \%$ ALA kwam overeen met het fluorescentie-niveau van een $2 \%$ ALA injectie vloeistof bij een identieke totale dosis. Na injectie van $2 \mathrm{mg}$ ALA of meer, bleek de door PDT geïnduceerde huidschade na belichting met $60 \mathrm{~J} \mathrm{~cm}^{-2}\left(100 \mathrm{~mW} \mathrm{~cm}^{-2}\right)$ significant groter dan na topicale applicatie van $20 \%$ ALA creme.

\section{HOOFDSTUK ACHT}

Behalve de toedieningswijze en de totaal toegediende dosis ALA, zou het uiteindelijke effect van PDT ook beinvloed kunnen worden door het toege- 


\section{BIBLIOGRAPHY}

Abide JM, Nahai F, Bennett RG. The meaning of surgical margins. Plast Reconstr Surg 1984;73:492-6.

Adam E. The technic of curettage surgery. J Am Acad Dermatol 1986;15:697-702.

Agarwal KC. Therapeutic actions of garlic constituents. Med Res Rev 1996;16:11124.

Allington HV. Liquid nitrogen in the treatment of skin diseases. Calif Med 1950;72: 153.

Altman DG. Practical statistics for medical research. Chapman \& Hall, London 1991.

Anders $\mathbf{M}$, Sport E, Krantz $H$ et al. Kryotherapie von malignen Lidtumoren. Ophthalmologe 1995;92:78-92.

Arnott $\mathbf{J}$. On the treatment of cancers by the regulated application of an anesthetic temperature. Churchill Livingstone, London, 1851.

Avril MF, Auperin A, Margulis A. Basal cell carcinoma of the face: surgery or radiotherapy? Results of a randomized study. Br J Cancer 1997;76:100-6.

Backer CL, Tinker JH, Robertson DM et al. Myocardial infarction following local anesthesia for ophthalmic surgery. Anaesth Anal 1980;59:257-62.

Bandieramonte G, Lepera P, Moglia D et al. Laser microsurgery for superficia! T1-T2 basal cell carcinoma of the eyelid margin. Ophthalmology 1997;104:1179-84.

Bastiaens MT, Hoefnagel JJ, Bruijn JA et al. Differences in age, site distribution, and sex between nodular and superficial basal cell carcinoma indicate different types of tumours. J Invest Dermatol 1998;110:880-4.

Bauer M, Loosli RM, Anderl $H$ et al. Operative Behandlung maligner Epitheliome der Haut. Grundsätze, Methoden, Ergebnisse. Chirurg 1977;48:170-9.

Beard C. Observations on the treatment of basal cell carcinoma of the eyelids. Trans Am Acad Ophthalmol Otolaryngol 1975;79:664-70.

Bech $\mathbf{O}$, Berg $K$, Moan $J$ et al. The $p H$ dependency of protoporphyrin $L X$ formation in cells incubated with 5-aminolevulinic acid. Cancer Lett 1997;113:25-9.

Bennett JP. From noli-me-tangere to rodent ulcer: The recognition of basal cell carcinoma. Br J Plast Surg 1974;27:144-54.

Betti $\mathbf{R}$, Inselvini $E$, Gualandri $L$ et al. Basal cell carcinoma of the auricular region: a study of 23 cases. I Dermatol 1995;22:655-8.

Beuchner SA. Intralesional interferon alfa- $2 b$ in the treatment of basal cell carcinoma. J Am Acad Dermatol 1991;24:731-4.

Beutner KR, Geisse $J K_{y}$ Helman $D$ et al. Therapeutic response of basal cell carcinoma to the immune response modifier imiquimod $5 \%$ cream. I Am Acad Dermatol 1999;41:1002-7.

Beuttner PG, Raasch BA. Incidence rates of skin cancer in Tounswille, Australia. Int $J$ Cancer 1998;78:587-93.

Bigby M. Evidence-Based Medicine in a nutshell. Arch Dermatol 1998;134:1609-18.

Biro $\mathbf{L}$, Price E, Brand A. Cryosurgery for basal cell carcinoma of the eyelid and the nose:five year experience. $J$ Am Acad Dermatoi 1982;6:1042-7.

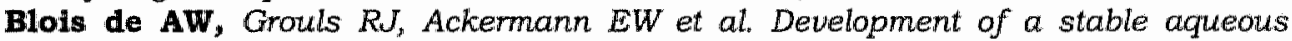
solution of 5-aminolevulinic acid for intracutaneous injection in photodynamic therapy (submitted).

Blois de $\mathbf{A W}$, Thissen MR, de Briajn HS et al. In wivo pharmacokinetics of protoporphyrin $I X$ accumulation following intracutaneous injection of 5-aminolevulinic acid (submitted).

Bonvallot T, Raulo $Y$, Zeller $J$ et al. Les epitheliomas baso-cellulaires des paupieres. Ann Dermatol Venereol 1988;115:669-78. 
paste belichtingsschema, waarbij zowel de lichtdosis, als de lichtintensiteit en het eventueel fractioneren van de belichting een rol kunnen spelen. Bij dit laatste aspect zou in de tijd tussen twee belichtingen opnieuw ALA in PpIX kunnen worden omgezet en zou tevens het tumorweefsel opnieuw van (voor PDT benodigd) zuurstof kunnen worden voorzien. Deze drie componenten van de belichting (dosis $3-60 \mathrm{~J} \mathrm{~cm}^{-2}$; intensiteit $50-100 \mathrm{~mW} \mathrm{~cm}$; enkele versus dubbele belichting) werden eveneens in een diermodel op gezonde huid onderzocht voor een standaard hoeveelheid intracutaan geïnjecteerd ALA $(0.5 \mathrm{ml} / 2 \%)$ hetgeen wederom vergeleken werd met de topicale toepassing, zoals beschreven in het laatste hoofdstuk van dit proefschrift. Ook hier werd gekeken naar de fluorescentie kinetiek van PpIX voor, na en tussen een gefractioneerde belichting, en werd de uiteindelijke huidschade na PDT visueel en histologisch bepaald.

Het bleek dat de hersynthese van PpIX na de eerste belichting nadelig beinvloed wordt door een stijgende lichtdosis. De uiteindelijke, door PDT geinduceerde schade nam toe bij toenemende lichtdosis, maar bleek niet beinvloed te worden door de lichtintensiteit. De gefractioneerde belichting, waarbij de totale lichtdosis in twee gelijke fracties werd gegeven met een belichtingsvrij interval van 2 uur, leidde uiteindelijk tot een significant grotere schade dan de enkelvoudige belichting met gelijke totale lichtdosis. Het effect met de topicale toepassing was in alle gevallen significant kleiner dan na intracutane toediening van ALA.

Op grond van de resultaten van de beide in vivo studies (hoofdstuk 7 en 8) zal de effectiviteit van PDT met intratumoraal geïnjecteerd ALA, gevolgd door een gefractioneerd belichtingsschema, in de toekomst verder onderzocht. worden bij patiënten met nodulaire BCC's. 
Borel DM. Cutaneous basosquamous carcinoma. Review of the literature and repont of 35 cases. Arch Pathol 1973;95:293-7.

Boring CC, Squires TS, Tong T et al. Cancer statistics 1994. CA 1994;44:7-26.

Brenner $\mathbf{s}$, Wolf $R$, Dascula DI. Topical tretinoin treatment in basal cell carcinoma. J Derm Surg oncol 1993; 19:264-6.

Breuningex $\mathbf{H}$, Rassner $G$, Undeutsch $W$. Operative Behandlung von Basaliomen mit errechnetem Sicherheitsabstand und histologischer Randcontrolle. Hautarzt 1984; 35:303-7.

Breuninger H. Histologic control of excised tissue edge in the operative treatment of basial cell carcinomas. I Dermatol Surg Oncol 1984;10:724-8.

Breuninger $\mathbf{H}$, Rassner $G$, Schaumberg-Lever $G$. Langzeiterfahrungen mit der Technik der histologische Schnittrandcontrolle (3-D Histologie). Hautarzt 1989;40:14-8.

Breuninger $\mathbf{H}$, Schippert $W$, Black $B$ et al. Untersuchungen zum Sicherheitsabstand und zur Exzisionstiefe in der operativen Behandlung von Basaliomen. Anwendung der dreidimensionalen histologischen Untersuchung bei 2016 Tumoren. Hautarzt $1989 ; 40: 693-700$.

Breuninger $\mathbf{H}_{s}$ Dietz $K$. Prediction of subclinical tumor infiltration in basal cell carcinoma. I Dermatol Surg Oncol 1991;17:574-8.

Breuninger $\mathbf{H}$, Dietz $K$, Rassner $G$. Das subklinische Infiltrationverhaiten von Basaliomen. Akt Dermatol 1992;18:129-32.

Breuninger $\mathbf{H}$, Anargyrou S. Kann die 20 MHz-Sonographie die subklinische Infiltration von malignen epithelialen Tumoren praetherapeutisch erkennen ? ZGI Hautkr 1993;162:196.

Breuninger $\mathbf{H}$, Holzschuh J. Die luckenlose histologische Darstellung der Schnittrander eines Hauttumorexisates (3D-Histologie) in einer Schnittebene mittels der "Fiundertechnik". Akt Dermatol 1994;20:7-10.

Breuninger $\mathbf{H}$. Micrographic surgery of malignant skin tumors: a comparison of the frozen technique with paraffin sectioning. Facial Plast Surg 1997;13:79-82.

Bruyn de HS, van der Veen $N$, Robinson $D J$, et al. Improvement of systemic 5aminolevulinic acid-based photodynamic therapy in vivo using light fractionation with a 75-minute interval. Cancer Res 1999;59:901-4.

Bullock JD, Beard C, Sullivan JH. Cryotherapy of basal cell carcinoma in oculoplastic surgery. Am. J Ophthalmol 1976;82:841-7.

Caccialanza M, Piccinno $R$, Beretta $M$ et al. Results and side effects of dermatologic radiotherapy: a retrospective study of irradiated cutaneous epithelial neoplasms. $J$ Am Acad Dermatol 1999;41:589-94.

Cairnduff $\mathbf{F}$, Stringer $M R$, Hudson $E J$, et alsuperficial photodynamic therapy with topical 5-aminolevulinic acid for superficial primary and secondary skin cancer. $B r J$ Cancer 1994;69:605-8.

Calzavara-Pinton PG. Repetitive photodynamic therapy with topical d-aminolevulinic acid as an appropriate approach to the routine treatment of superficial non-melanoma skin cancer. J Photochem Photobiol B:Biol. 1995;29:53-57.

Caquoin C, Bougard C. In: Wolf $J$. Die Lehre von der Krebskrankheiten. Jehna, Fischer 1913;3:107.

Casas A Fukuda $H$, Meiss $R$ et al. Topical and intratumoural photodynamic therapy with 5-aminolevulinic acid in a subcutaneous murine mammary adenocarcinoma. Cancer Lett 1999;141:29-38.

Chuang TY, Popescu A, Su WP et al. Basal cell carcinoma: a population based incidence study in Rochester, Minnesota. J Am Acad Dermatol 1990;22:413-7.

Chuang TY, Reizner GT, Elpern $D J$ et al. Nonmelanoma skin cancer in Japanese ethnic Hawaiians in Kauai, Hawait: an incidence report. J Am Acad Dermatol 1995; 
33:422-6.

Coebergh JW, Neumann HA, Vrints LW et al. Thends in the incidence of nonmelanoma skin cancer in the SE Netherlands 1975-1988; a registry-based study. Br $J$ Dermatol 1991; 125:353-9.

Coebergh JW. Non-melanoma skin cancer. In: Coebergh JW, wan der Heijden $L H$, Janssen-Hejinen ML. Cancer Incidence and Survival in the Southeast of the Netherlands 1955-1994, Eindhoven, IKZ, 51-3.

Cook J, Zitelli JA. Mohs micragraphic surgery: a cost analysis. JAAD 1998:39:698703.

Cooper IS. Cryogenic surgery: A new method of destruction or extirpation of benign or malignant tissues. $N$ Engl $J$ Med 1963:268:743.

Cutler s, Ederer F. Maximum utilization of the life table method in analyzing survival. I Chronic Dis 1958;8:699-712.

Daniell MD, Hill JS. A history of photodynamic therapy. Aust $N Z$ I Surg 1991; $61: 340-8$.

Davis MM, Hanke $C W$, Zollinger $T W$ et al. Skin cancer in patients with chronic radiation dermatitis. J Am Acad Dermatol 1989;20:608-16.

D'Errico M, Calcagnile AS, Corona $R$ et al. P53 mutations and chromosome instability in basal cell carcinomas developed at an early or late age. Cancer Res 1997;57: 747-52.

Dhir A, Orengo $I_{s}$ Bruce $S$ et al. Basal cell carcinoma on the scalp of an Indian patient. Dermatol Surg 1995;21:247-50.

Dinehart SM, Dodge $R$. Stanley WE et al. Basal cell carcinomas treated with Mohs' surgery. A comparison of 54 younger patients with 1050 older patients. I Dermatol Surg Oncol 1992;18:560-6.

Dobbinson S, Peipers $A$, Reading $D$. A national approach to skin cancer prevention: the National SunSmart Schools Program. Med J Aust 1998;169:513-4.

Domarus von HV, Stevens PJ. Metastatic basal cell carcinoma: report of five cases and review of 170 cases in the literature. I Am Acad Dermatol 1984;10:1043-60.

Dougherty TJ, Grindy GB, Weishaupt KR, et al. Photoradiation therapy II. Cure of animal tumours with haematoporphyrin and light. I Natl Cancer Inst 1975;55:11 15-21.

Dougherty TJ, Kaufman JE, Goldfarb A et al. Photoradiation therapy for the treatment of malignant tumours. Cancer Res 1978;38:2628-35.

Dougherty TJ, Gomer CJ, Henderson BW et al. Photodynamic therapy. I Natl Cancer Inst 1998;90:889-905.

Dunn JE Jr, Levin EA, Linden $G$ et al. Skin cancer as a cause of death. Calif Med 1965;102:301-63.

Edens BL, Bartlow GA, Haghighi $P$ et al. Effectiveness of curettage and electrodesiccation in the removal of basal cell carcinoma. J Am Acad Dermatol 1983;9:383-8.

Egger M, Smith GD. Meta-analysis; potentials and promise. Br Med J 1997;314: $1371-4$.

Egger M, Smith GD, Philips AN. Meta-analysis; principles and procedures. Br Med $J$ 1997;315:1533-7.

Egger M, Smith GD. Meta-analysis; bias in location and selection of studies. $\mathrm{Br}$ Med $J$ $1998 ; 316: 61-6$.

Elfsson B, Wallin $I$, Eksborg $S$ et al. Stability of 5-aminolemilinic acid in aqueous solution. Eur J Pharm Sci 1998;7:87-91.

Elinitsas $\mathbf{R}$, Belle $v P$, Elder $D$. Laboratory methods. In: Elder $D$, Elinitsas $R$, Jaworsky $C$ et al. Lever's histopathology of the skin, 8th eds, Lippincott-Raven 1997, Philadelphia. Ch 4; 51-60.

Emmett AJ. Surgical analysis and biological behaviour of 2277 basal cell carcino- 
mas. Aust $N Z$ J Surg 1990;60:855-63.

English DR, Kricker $A$, Heenan $P J$ et al. Incidence of non-melanoma skin cancer in Geraldton, Western Australia. Int J Cancer 1997;73:629-33.

Euvrard s, Kanitakis J, Pouteil-Noble C et al. Comparative epidemiologic study of premalignant and malignant epithelial cutaneous lesions developing after kidney and heart transplantation. J Am Acad Dermatol 1995;33:222-9.

Faber WR, Rampen FJ. Cryosurgical treatment of malignant skin tumours. Ned Tijdschr Geneeskd 1986;130:918-21.

Fijan $\mathbf{s}$, Honigsmann $H$, Ortel $B$. Photodynamic therapy of epithelial skin tumours $u-$ sing delta-aminolevulinic acid and desferrioxamine. Br J Dermatol 1995;133:282-8.

Fingar VH, Henderson BW. Drug and light dose dependence of photodynamic therapy: a study of tumour and normal tissue response. Photochem Photobiol 1987;46: $837-41$

Fingar VH, Wieman TJ, Wiehle SA et al. The role of microvascular damage in photodynamic therapy: the effect of treatment on vessel constriction, permeability and leucocyte adhesion. Cancer Res 1992;52:4914-21.

Fink-Puches $\mathbf{R}$, Wolf $P$, Kerl $H$ et al. Photodynamic therapy of superficial basal cell carcinoma by instillation of aminolevulinic acid and irradiation with visible light. Arch Dermatol 1997; 133:1494-5.

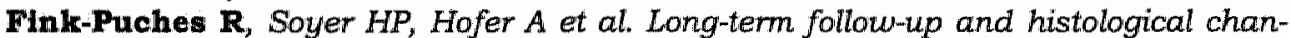
ges of superficial nonmelanoma skin cancers treated with topical d-aminolevulinic acid photodynamic therapy. Arch Dermatol 1998;134:821-6.

Finsen NR. Phototherapy. London, 1901.

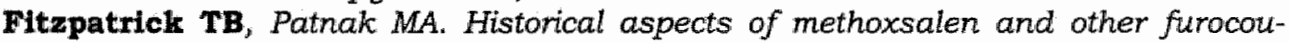
marins. J Invest Dermatol 1959;23:229-31.

Fitzpatrick P, Thompson GA, Easterbrook WM et al. Basal and squamous cell carcinoma of the eyelids and their treatment by radiotherapy. Int $J$ Radiol OncoBiol Phys 1984;10:449-54.

Foote CS. Chemical mechanism of photodynamic action. Proc SPIE Institute "Advanced Optical Technologies on Photodynamic Therapy" 1990;6:1 15-26.

Foster TH, Murant RS, Bryant RS et al. Oxygen consumption and diffusion effect in photodynamic therapy. Radiat Res 1990;126:296-303.

Franceschi S, Dal Maso L, Amiani S et al. Risk of cancer other than Kaposi's sarcoma and non-Hodgkin's lymphoma in persons with AIDS in Italy. Cancer and AIDS Registry Linkage Study. Br J Cancer 1998;78:966-70.

Fraunfelder FT, Zacarian SA, Wingfield DL et al. Results of cryotherapy for eyelid malignancies. Am J Ophthalmol 1984;97:184-8.

Fritsch C, Batz $J$, Bolsen $K$. Ex-vivo-application of d-aminolevulinic acid induces high and specific porphyrin levels in human skin tumours; possible basis for selec-tive photodynamic therapy. Photochem Photobiol 1997;66:114-8 .

Fritsch C, Goertz G, Ruzicka T. Photodynamic therapy in dermatology. Arch Dermatol $1998 ; 134: 207-14$.

Fuchs $\mathrm{J}$, Thiele $J$ : The role of oxygen in cutaneous photodynamic therapy. Free Radic Biol Med 1998;24:835-847.

Gage AA. Cryosurgery for skin disease: Variants in technique. In: Epstein E. Controversies in Dermatology. WB Saunders Co., Philadelphia 1984;151-60.

Gallagher RP, Bajdik CD, Fincham S et al. Chemical exposures, medical history, and risk of squamous and basal cell carcinoma of the skin. Cancer Epidemiol Biomarkers Prev 1996;5:419-24.

Germann G, Bemstein-Sommer B, Petrovici V et al. Differenzierte, onkologische adäquate Therapie des Basalioms. Handchir Mikrachir Plast Chir 1992,24:151-8. 
Gezondheidsraad: Commissie Risico's UV-straling. Advies: "UV-straling uit zonlicht". Gezondheidsraad, Den Haag, 1994: publicatie nr 1994/05.

Gilaberte $\mathbf{Y}$, Pereboom D, Carapeto $F J$ et al. Flow cytometry study of the role of superoxide anion and hydrogen peroxide in cellular photodestruction with 5-aminolevulinic acid-induced protoporphyrin IX. Photodermatol Photoimmunol Photomed 1997; 13:43-9.

Glatt HJ, Olson JJ, Putterman AM. Conventional frozen sections in periocular basal cell carcinoma: a review of 236 cases. Ophthalmic Surg 1992;23:6-8.

Gloster HM Jr, Brodland DG. The epidemiology of skin cancer. Dermatol Surg 1996; 22:217-26.

Goldberg DP. Assessment and surgical treatment of basal cell carcinoma. Clin Plast Surg 1997;24:673-86.

Gorlin R. Nevoid basal cell carcinoma syndrome. Dermatol Clin 1995;13:113-25.

Grant WE, Hopper C, MacRobert AJ, et al. Photodynamic therapy of oral cancer; photosensitization with systemic aminolevulinic acid. Lancet 1993;342:147-8.

Green A, Battistutta D. Incidence and determinants of skin cancer in a high risk Australian population. Int J Cancer 1990;46:356-61.

Green A, Battistutta $D$, Hart $V$ et al. Skin cancer in a subtropical Australian population: incidence and lack of association with occupation. The Nambour Study Group. Am J Epidemiol 1996;144:1034-40.

Greenway HT, Comell RC, Tanner DU et al. Treatment of basal cell carcinoma with intralesional interferon. $J$ Am Acad Dermatol 1986; 15:437-43.

Grossman L, Wei Q. DNA repair and epidemiology of basal cell carcinoma. Clin Chem 1995;41:1854-63.

Gunnarson G, Larko $O$, Hersle $K$. Cryosurgery of eyelid basal cell carcinomas. Acta Ophthalmol 1990;68; 241-5.

Halder RM, Bridgeman-Shah S. Skin cancer in African Americans. Cancer 1995; 75 : $667-73$.

Hannuksela-Svahn A, Pukkala E, Karvonen J. Basal cell skin carcinoma and other non-melanoma skin cancers in Finland from 1956 through 1995. Arch Dermatol $1999 ; 135: 781-6$.

Harth $\mathbf{Y}$, Hirsgowitz B, Kaplan B. Modified topical photodynamic therapy of superficial skin tumours, utilizing aminolevulinic acid, penetration enhancers, red light and hyperthermia. Dermatol Surg 1998;24:723-6.

Henderson BW, Dougherty TU: How does photodynamic therapy work? Photochem Photobiol 1992;55:145-157.

Herman MA, Webber $J$, Fromm $D$ et al. Hemodynamic effects of 5-aminolevulinic acid in humans. I Photochem Photobiol B: Biol 1998;43:61-5.

Hiesse C, Rieu $P$, Kriaa $F$ et al. Malignancy after renal transplantation: analysis of incidence and risk factors in 1700 patients followed during a 25-year period. Transplant Proc 1997;29:831-3.

Hilligersberg $\vee \mathbf{R}$, Berg $v d J W$, Kort $W J$ et al. Selective accumulation of endogenously produced porphyrins in a liver metastasis model in rats. Gastroenterology $1992 ; 103: 647-51$.

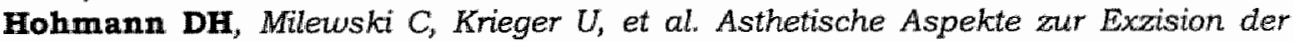
Basaliome im kopf-und halsbereich. Laryngorhinootologie 1992;71:311-4.

Holmkvist KA, Rogers GS, Dahl P. Incidence of residual basal cell carcinoma in patients who appear tumor free after biopsy. I Am Acad Dermatol 1999;41:600-5.

Holt PJ, Motley RJ. The treatment of basal cell carcinoma. Curr Pract Surg 1994;6: 98-101. 
Hua $\mathbf{z}$, Gibson SL, Foster TH, et al. Effectiveness of d-aminoleulinic acid-induced protoporphyrin as a photosensitizer for photodynamic therapy in vivo. Cancer Res 1995;55:1723-31.

Hughes JR, Marks $R$, Pearse $A D$ et al. Clinical response and tissue effect of etretinate treatment of patients with solar keratosis and basal cell carcinoma. J Am Acad Dermatol 1988;18:522-9.

Hughes $\mathbf{J R}$, Higgins EM, Smith $J$ et al. Increase in non-melanoma skin cancer -the King's College Hospital experience 1970-1992. Clin Exp dermatol 1995;20:304-7.

Humphreys TR, Mathotra $R$, Scharf MV et al. Treatment of superficial basal cell carcinoma and squamous cell carcinoma in situ with a high-energy pulsed carbon dioxide laser. Arch Dermatol 1998; 134:1247-52.

Hurlimann AF, Hanggi $G$, Panizzon RG. Photadynamic therapy of superficial basal cell carcinomas using topical 5-aminolevulinic acid in a nanocolloid lotion. Dermatology 1998; 197:248-254.

Ichihashi $\mathbf{M}$, Nanuse $K$, Harada $S$ et al. Trends in nonmelanoma skin cancer in Japan. Recent Results Cancer Res 1995;139:253-73.

Iinuma $\mathbf{S}$, Farshi SS, Oetel B, et al. A mechanistic study of cellular photodestruction with 5-aminole rulinic acid-induced porphyrin. Br J Cancer 1994;70:21-8.

Jacob A. Observations respecting an ulcer of peculiar character which attacks eyelids and other parts of the face. Dubl Hosp Reprs 1827;4:232-9.

Jones CM, Mang $T H$, Cooper $M$ et al. Photodynamic therapy in the treatment of Bowen's disease. J Am Acad Dermatol 1992;27:979-82.

Jonna BP, Delfino RJ, Newman WG et al. Positive predictive value for presumptive diagnoses of skin cancer and compliance with follow-up among patients attending a community screening program. Prev Med 1998;27:611-6.

Julian CG, Bowers PW. A prospective study of Mohs' micrographic surgery in two English centres. Br J Dermatol 1997;136:515-8.

Juzenas $\mathbf{P}$, Sorensen $R$, lani $V$, et al. Uptake of topically applied 5-aminolevulinic acid and production of protoporphyrin. $L X$ in normal mouse skin: dependence on skin temperature. Photochem Photobiol 1999;69: 478-81.

Kaldor J, Shugg D, Young B et al. Non-melanoma skin cancer: ten years of cancerregistry-based surveillance. Int $J$ Dermatol 1993;53;886-91.

Karagas MR, Greenberg ER, Spencer SK et al. Increase in incidence rates of basal cell and siquamous cell skin cancer in New Hampshire, USA. New Hampshire Skin Cancer Study Group. Int $J$ Cancer 1999;81:555-9.

Kennedy JC, Pottier RH, Pross DC. Photodynamic therapy with endogenous protoporphyrin IX: basic principles and present clcinical experience. I Photochem Photobiol B: Biol. $1990 ; 6: 143-8$.

Kennedy JC, Pottier RH. Endogenous protoporphyrin $I X$, a clinically useful photosensitizer for photodynamic therapy. J Photochem Photobiol B: Biol 1992;14:275-92.

Kimonis VE, Goldstein $A M$, Pastakia $B$ et al. Clinical manifestations in 105 persons with nevoid basal cell carcinoma syndrome. Am. J Med Genet 1997;69:299-308.

Kirkup ME, de Berker DA. Clinical measurement of dimensions of basal cell carcinoma. effect of waiting for elective surgery. Br J Dermatol 1999;141:876-9.

Klinteberg C, Enejder AM, Wang I et al. Kinetic fluorescence studies of 5-aminolevulinic acid-induced protoporphyrin LX accumulation in basal cell carcinoma. $J$ Photochem Photobiol B: Biol 1999;49:120-8.

Koga $\mathbf{Y}$, Sawada Y. Basal cell carcinoma developing in a burn scar. Bums 1997; 23:75-7.

Kopf AW, Bart RS, Schrager D et al. Curettage-electrodesiccation treatment of basal cell carcinomas. Arch Dermatol 1977;113:439-43. 
Kopf AW. Computer analysis of 3531 basal cell carcinomas of the skin. I Dermatol (Tokyo) 1979;6:267-81.

Kopke LF, Konz B. Mikrographische Chinurgie. Eine methodische Bestandsaufnahme. Hautarat 1995;46:607-14.

Krekels GA, Verhaegh ME, Neumann HA. Mohs' micrografische chirurgie. In: Beljaards RC, Neumann HA. Oncologische dermatochinurgie. Vakgroep dermatologie, Universiteit Maastricht 1996(a); hfst 9:123-30.

Krekels GA, Verhaegh ME, Neumann HA. Mohs' micrografische chirurgie. NTVD 1996 (b);6:90-6.

Kricker A, Armstrong $B K$, English $D R$ et al. A dose-response curve for sun exposure and basal cell carcinoma. Int J Cancer 1995 (b);60:482-8.

Kricker A, Armstrong $B K$, English $D R$ et al. Does intermittent sun exposure cause basal cell carcinoma? A case-control study in Western. Australia. Int $J$ Cancer 1995 (a);60:489-94.

Krompecher E. Der Basalzellenkrebs. Jena, Gustave Fischer, 1903.

Kuflik EG, Gage A.A. Cryosurgical treatment for skin cancer. New York: Igaku-Shoin, 1990:35-51.

Kuflik EG, Gage AA. The five year cure rate achieved by cryosurgery for skin cancer. I Am Acad Dermatol 1991;24:1002-4.

Kune GA, Bannerman $S$, Field $B$ et al. Diet, alcohol, smoking, serum beta-carotene, and vitamin $A$ in male nonmelanocytic skin cancer patients and controls. Nutr Cancer $1992 ; 18: 237-44$.

Lang PG jr, Maize JC. Histologic study of recurrent basal cell carcinoma and treatment implications. J Am Acad Dermatol 1986;14:186-96.

Lang PG jr. Variables to consider in the management of non-melanoma skin cancer. $J$ Geriatr Dermatol 1996;4:231-7.

Langer S, Abels $C$, Botzlar A, et al. Active and higher intracellular uptake of 5 . aminolevulinic actd in tumors may be inhibited by glycine. I Invest Dermatol 1999; 112:723-8.

Launis $\mathbf{J}$. Curettage-electrodesiccation as a treatment for basal cell carcinomas. Meeting abstract Melanoma Res 1993;3:27.

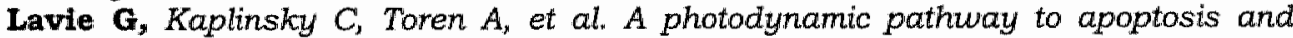
necrosis induced by dimethyltetrahydroxyhelianthrone and hypericin in leukaemic cells: possible releuance to photodnamic therapy. Br J Cancer 1999;79:423-32.

Lear JT, Smith AG, Bowers $B$ et al. Truncal tumor site is assocuated with high risk of multiple basal cell carcinoma and is influences by glutathione S-transferase, GSTT1, and cytochrome P450, CYPIA1 genotypes, and their interaction. $J$ Invest Dermatol 1997;108:519-22.

Leengoed $\mathbf{v} \mathbf{H L}$, Versteeg AA, van der Veen $N$ et al. Tissue localizing properties of some photosensitizers studied by in vivo flusorescence imaging. I Photochem Photobiol B:Biol 1990;6:111-9.

Leffel DJ, Headington JT, Wong DS et al. Agressive-growth basal cell carcinoma in young adults. Arch Dermatol 1991;127:1663-7.

Levi F, La Vecchia C, Van-Cong TE et al. Descriptive epidemiology of skin cancer in the Suriss canton of Vaud. Int $J$ Cancer 1988;42:811-6.

Lindelof B Sigurgeirsson B. PUVA and cancer: a large scale epidemiological study. Lancet $1991 ; 338: 91-3$.

Lindgren $\mathbf{G}$, Larko $O$. Long-term follow-up of cryosurgery of basal cell carcinoma of the eyelid. I Am Acad Dermatol 1997;36:742-6.

Lippert HD, Wiskemañ A. Röntgenbestrahlung von Basaliomen im Lidbereich. Bestrahlungstechnik und Ergebnisse. Hautarzt 1978;27:209-12. 
Lipson $\mathbf{R L}_{\text {, Baldes }} \mathrm{EJ}$. The photodynamic properties of a particular haematoporphyrin derivative. Arch Dermatol 1960;82:508-16.

Lipson RL, Baldes $E J$, Olsen AM. The use of a derivative of haematoporphyrin in tumour detection. J Natl Cancer Inst 1961;26:1-11.

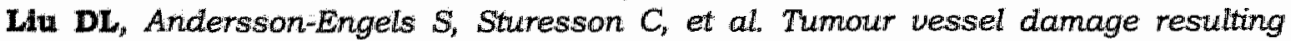
from laser-induced hyperthermia alone and in combination with photodynamic therapy. Cancer Lett 1997;111:157-65.

Lloyd Roberts D. Incidence of non-melanoma skin cancer in West Glamorgan, South Wales. Br J Dermatol 1990;122:399-403.

Lo JS, Snow SN, Reizner GT et al. Metastatic basal cell carcinoma: report of twelve cases with a review of the literature. J Am Acad Dermatol 1991;24:715-9.

Lock-Andersen J, Drzewiecki KT, Wulf HC. Eye and hair colour, skin type and constitutive skin pigmentation as risk factors for basal cell carcinoma and cutaneous malignant melanoma. A Danish case-control study. Acta Derm Venereol 1999;79:74-80.

Loh CS, Bedwell J, MacRobert AJ, et al. Photodynamic therapy of the normal rat stomach; a comparative study between disulphonated aluminium phthalocyanine and 5-aminoleutinic acid. BrJ Cancer 1992;66:452-62.

Loh CS, MacRobert $A J$, Bedwell $J_{1}$ et al. Oral versus intravenous administration of 5aminolevulinic acid for photodynamic therapy. Br J Cancer 1993 (a);68:41-51.

Loh CS, Vernon DI, MacRobert AJ, et al. Endogenous porphyrin distribution induced by 5-aminolevulinic acid in the tissue layers of the gastro-intestinal tract. $J$ Photo chem Photobiol B: Biol 1993 (b);20:47-54.

Lucke TW, Hole DJ, Mackie RM. An audit of the completeness of non-melanoma skin cancer registration in Greater Glasgow. Br J Dermatol 1997;137:761-3.

Luis R, del Carmen M, Robledo M, et al. Multiple hereditary infundibulocystic basal cell carcinomas. Arch Dermatol 1999;135:1227-35.

Mahmoud SF, Azadeh B. Basal cell carcinoma in Qatar. Int J Dermatol 1996;35:7046.

Mallik Z, Lugaci $H$. Destruction of erytroleukemic cells by photoactivation of endogenous porphyrins. Br J Cancer 1987;56:589-95.

Marchac D, Papadopulos 0, Duport $G$. Currative and aesthetic results of surgical treatment of 138 basal cell carcinomas. J Dermatol Surg Oncol 1982;8:379-87.

Marghoob AA. Basal and squamous cell carcinomas. What every primary care physician should know. Postgr Med 1997; 102:139-59.

Marks $\mathbf{R}$, Jolley $D$, Lectsas $S$ et al. The role of childhood exposure to sunlight in the development of solar keratosis and non-melanocytic skin cancer. Med $J$ Aust $1990,152: 62-6$.

Marks $\mathbf{R}$. An overview of skin cancers: incidence and causation. Cancer 1995; $75: 607-12$.

Marks R. Two decades of the public health approach to skin cancer control in Austratia: why, how and where are we now? Australas $J$ Dermatol 1999;40;1-5.

Marmelzat wL. "Noli-me-tangere" circa 1754: Jacques Daviel's forgotten contribution to skin cancer. Arch Dermatol 1964;90:280-3.

McCormack CJ, Kelly JW, Dorevitch AP. Differences in age and body site distribution of the histological subtypes of basal cell carcinoma. Arch Dermatol 1997; 133:593-6.

McDaniel WE. Therapy for basal cell carcinomas by curettage only. Further study. Arch Dermatol 1983;119:901-3.

McKnight CK, Magnusson B. Tumors in Iceland. In: Malignant tumars of the skin: a histologic classification. Acta Pathol Microbiol Scand 1979;87:37-44.

Mehregan AH, Pinkus H. Life history of organoid nevi. Arch Dermatol 1965;91:574- 
88.

Meijnders PJ, Star WM, de Bruijn HS, et al. Clinical results of photodynamic therapy for superficial skin malignancies or actinic keratosis using topical 5-aminolevilinic acid. Lasers Med Sci. 1996;11:123-31.

Messmann H, Mlkoy $P$, Buonaccorsi $G$, et al: Enhancement of photodynamic therapy with 5-aminolevulinic acid-induced porphyrin photosensitisation in normal rat colon by threshold and light fractionation studies. Br J Cancer 1995;72:589-94.

Meyer W, Schwarz $R$, Neurand $K$. The skin of the domestic mammals as a model for the human skin, with special reference to the domestic pig. Curr Prob Dermatol 1978;7:39-52.

Meyer w. Bemerkungen zur Eignung der Schweinehaut als biologisches Modell fur die Haut des Menschen. Hautarzt 1996;47:178-82.

Meyer-Betz F. Untersuchungen uber die biologische (photodynamische) Wirkung des Hematoporphyrins und anderer Derivative des Blut-und-Gallenfarbstoffs. Dtsch Arch Klin Med 1913; 1 12:476-503.

Michaelsson G, Olsson $E$, Westermark $P$. The Rombo syndrome: a familial disorder with vermiculate atrophoderma, milia, hypotrichosis, trichoepitheliomas, basal cell carcinomas, and peripheral vasodilatation with cyanosis. Acta Derm Venereol 1981; 61:479-503.

Miller DL, Weinstock MA. Nonmelanoma skin cancer in the United States; incidence. J Am Acad Derm 1994;30:774-8.

Miller LJ, Argenyi $Z B$, Whitaker $D C$. The preparation of frozen sections for micrographic surgery. I Dermatol Surg Oncol 1993;19:1023-9.

MMWR. Sun-protection behaviors used by adults for their children-United States, 1997. Morb Mortal Wkly Rep 1998;47:480-2.

Moan J. Effect of bleaching of porphyrin sensitizers during photodynamic therapy. Cancer Lett 1986;33:45-53.

Moan J. On the diffusion length of singlet oxygen in cells and tissues. $J$ Photochem Photobiol B:Biology 1990;6:343-4.

Moan J, Iani $V, M a L W$, et al. Photodegradation of sensitizers in mouse skin during PCT. Proc SPIE 1995;2625:187-93.

Mohs FE. Chemosurgery. Microscopically controlled surgery for skin cancer. Charles C. Thomas, Springfield, II, 1978;1-29.

Mohs FE. Micrographic surgery for the microscopically controlled excision of eyelid cancers. Arch Ophthalmol 1986;104:901-9.

Mohs F, Larson P, Iriondo M. Micrographic surgery for the microscopically controlled excision of carcinoma of the extemal ear. I Am Acad Dermatol 1988;19:729-37.

Morton CA, Mackie RM. Photodynamic therapy for basal cell carcinoma: effect of tumour thickness and duration of photosensitizer application on response. Arch Dermatol 1998; 134:248-9.

Motley RJ, Holt PJ. A simple device for optimal tissue preparation for Mohs" micrographic surgery. Br J Dermatol 1992; 126:57-9.

Motley RJ. The treatment of basal cell carcinoma. $J$ Derm Treatment 1995;6:121-5.

Nagano T, Ueda M, Suzuki $T$ et al. Skin cancer screening in Okinawa, Japan. I Dermatol Sci 1999;19:161-5.

Naylor CD. Two cheers for meta-analysis: problems and opportunities in aggregating results of clinical trials. Can Med Assoc 1988;138:891-5.

Naylor CD. Meta-analysis and the meta-epidemiology of clinical research. $\mathrm{Br} \mathrm{Med} J$ 1997;315:617-9.

Neumann HA. Approach to the oncological patient. In: Beljaards RC, Neumann HA. Oncological dermatosurgery 1996 (a), University of Maastricht, the Netheriands. Ch 
$11 ; 135-46$.

Neumann HA. Histological verification of skin tumours, In: Beljaards $R C_{t}$ Neumann HA. Oncological dermatosurgery 1996 (b), University of Maastricht, the Netherlands. Ch 2:40-3.

Neumann HA, Krekels GA, Verhaegh ME. Treatment of 208 extensive basal cell carcinoma with Mohs' micorgraphic surgery. I Eur Acad derm Venereol 1996;6:217-25.

Noodleman FR, Pollack SV. Trauma as a possible etiologic factor in basal cell carcinoma. $J$ Dermatol Surg Oncol 1986;12:841-6.

Noodt BB, Berg $K$, Stokke $T$, et al. Apoptosis and necrosis induced with light and 5aminoleutinic acid-derived protoporphyrin IX. Br J Cancer 1996;74;22-9.

Nordin $\mathbf{P}$, Larko 0 , Stenquist B. Five-year results of curettage-cryosurgery of selected large primary basal cell carcinomas on the nose: an altemative treatment in a geographical area underserved by Mohs' surgery. Br J Dermatol 1997;136:180-3.

Novo M, Huttmann G, Diddens $H$ et al. Chemically instability of 5-aminolevulinic acid used in the fluorescence diagnosis of bladder tumours. I Photochem Photobiol B: Biol $1992 ; 34: 143-8$.

Orenstein A, Kostenich $G$, Tsur $H$ et al. Photodynamic therapy of human skin tumours using topical application of 5-aminolevulinic acid, DMSO and EDTA. In: Brault $D$, Jori G, Moan $J$ et al. Photodynamic therapy of cancer $I$. Proc SPIE 1995, val $2325 ; 100-5$.

Orenstein A, Kostenich $G$, Kopolovic $Y$, et al. Enhancement of ALA-PDT damage by IR-induced hyperthermia on a colon carcinoma model. Photochem Photobiol 1999;69: 703-7.

Osterlind A, Hor Jenssen $K$, MollerJenssen $O$. Incidence of cutaneous malignant melanoma in Denmark 1978-82. Anatomic site-distributions, histological types and comparison with non-melanoma skin cancer. Br J Dermatol 1988;58:385-91.

Panje WR, Ceilley RI. The influence of embryologic of the midface on the spread of epithelial malignancies. Laryngoscope 1979;89:1914-20.

Parker SL, Tong T, Bolden S et al. Cancer statistics 1996. CA 1996:46:5-27.

Parmar MK, Machin D. Survival curves. In: Survival Analysis, a practical approach Wiley \& Sons, Chichester 1995;21-51.

Peng $\mathbf{Q}$, Warloe $T$, Moan $J$ et al. Distribution of 5-aminolevulinic acid induced porphyrins in nodulo-ulcerative basal cell carcinoma. Photochem Photobiol 1995;62:906-13.

Peng Q, Moan $J$, Nesiand $J M$. Correlation of subcellular and intratumoral photosensitizer localization with uitrastructural features after photodynamic therapy. Ultrastruct Pathol1996;20:109-29.

Peng $\mathbf{Q}$, Moan $J$, Warloe $T$ et al. 5-Aminoleuulinic acid-based photodynamic therapy; clinical research and future challenges. Cancer 1997;79:2282-308.

Petrovich Z, Parker $R G_{i}$ Luxton $G$ et al. Carcinoma of the lip and selected sites of head and neck skin. A clinical study of 896 patients. Radiother Oncol 1987;8:11-7.

Plcoto AM. Picoto A. Technical procedures for Mohs fresh tissue surgery. I Dermatol Surg Oncol $1986 ; 12: 134-8$.

Pierard-Franchimont $\mathbf{C}$, Uhoda $I$, Pierard GE. Cutaneous cancers in the Mosan region and Ardennes of Belgium. Dermatology 1999;198:187-91.

Plosila M, Küstala $R$, Niemi K-M. The Bazex syndrome: Follicular atrophoderma with multiple basal cell carcinomas, hypotrichosis, and hypohidrosis. Clin Exp Dermatol 1981;6:31-41.

Preston DS, Stem RS. Nonmelanoma cancers of the skin. N Engl I Med 1992; 327:1649.62.

Pogue BW, Hasan T. A theoretical study of light fractionation and dose-rate effects in photodynamic therapy. Radiat Res 1997;147:551-9. 
Raab 0. Ueber die Wirkung Fluorescierenden Stoffe auf Infusorie. $Z$ Biol 1900,39: 524-46.

Ramani ML, Bennett RG. High prevalence of skin cancer in World War II servicemen stationed in the pacific theater. IAm Acad Dermatol 1993;28:733-7.

Rampen FH, Neumann HA, Kiemeney LA. Fundamentals of skin cancer/melanoma screening campaigns. Clin Exp Dermatol 1992;17:307-12.

Rand RW, Rinfret AP, von Leden $H$. In: Cryosurgery. Thomas, Springfield, Mlinois, $1968 ; 96$.

Randle HW, Roenigk RK. Characteristics of giant basail cell carcinoma. In: Roenigk RK, Roenigk HH Jr Eds. Surgical dermatology: Advances in Current Practice, St Louis, Mosby 1993:93-100.

Randle HW. Basal cell carcinoma. Identification and treatment of high-risk patient. Dermatol Surg 1996;22:255-61.

Reizner GT, Chuang TY, Elpern DJ et al. Basal cell carcinoma in Kauai, Hawaii: the highest documented incidence in the United States. $J$ Am Acad Dermatol 1993;29: 184-9.

Reymann F, Kopp $H$. Treatment of basal cell carcinoma of the skin with ultrasoft $X$ rays. Dermatologica 1978;156:40-7.

Reymann F. Treatment of basal cell carcinoma of the skin with 5-fluorouracil aintment. A 10-year follow-up study. Dermatologica 1979;158:368-72.

Reymann F. Basal cell carcinoma of the skin; recurrence rate after different types of treatment. Dermatologica 1980;161:217-26.

Rick K, Sroka $\boldsymbol{R}$, Stepp $H$ et al. Pharmacokinetics of 5-aminolevulinic acid-induced protoporphyrin $I X$ in skin and blood. J Photachem Photobiol B: Biol 1997;40:313-9.

Rigel DS, Friedman RJ, Kopf AW et al. Lifetime risk for the development of skin cancer in the US populations: current estimate is now 1 in 5. J Am Acad Dermatol 1996; $35: 1012-27$.

Roberts WG, Smith KM, Loomis $M$ et al. Skin photosensitivity and photodestruction of several potential photosensitizers. Photochem Photobiol 1989;49:431-8.

Roberts DJ, Cairnduff $F$, Driver I et al. Tumour vascular shutdown following photodynamic therapy based on polyhaematoparphyrin or 5-aminolevulinic acid. Int $J$ Oncol $1994 ; 5: 763-8$.

Robins P. Chemosurgery: My 15 years of experience. I Dermatol Surg Oncol 1984;7: 779-89.

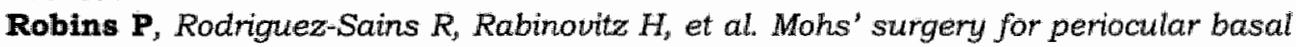
cell carcinomas. J Dermatol Surg Oncol 1985;11:1203-7.

Robinson DJ, de Bruyn HS, Van der Veen $N$, et al. Fluorescence photobleaching of ALA-induced protoporphyrin $I X$ during photodynamic therapy of normal hairless mouse skin; the effect of light dose and irradiance and the resulting biological effect. Photochem Photobiol 1998;67:140-9.

Robinson DJ, Vernon DI, de Bruijn HS, et al. Photo-inactivation of ferrochelatase and the kinetics of PpLX-fluorescence following ALA-PDT in vivo. Photochem Photobiol 1999;69:39S, Abstract MPM-E12.

Robinson JK. Mohs' micrographic surgery. Clin. Plast Surg 1993:20;149-56.

Rhodes AR. Public education and cancer of the skin. What do people need to know about melanoma and nonmelanoma skin cancer? Cancer 1995;75:613-36.

Rhodes LE, Tsoukas MM, Andersion RR et al. Iontophoretic delivery of ALA provides a quantitative model for ALA pharmacokinetics and PPIX phototoxicity in human skin. $J$ Invest Dermatol 1997;108:87-91.

Ron E, Preston DL, Kishikawa $M$ et al. Skin tumor risk among atomic-bomb survivors in Japan. Cancer Causes Control 1998;9:393-401. 
Rowe DE, Carroll RJ, Day CL JT. Lang term recurrence rates in previously untreated (primary) basal cell carcinoma: implications for patient follow-up. I Dermatol Surg Oncol 1989; 15:315-28.

Rowe DE, Carroll RJ, Day CL. Mohs surgery is the treatment of choice for recurrent (previously treated) basal cell carcinoma. J Dermatol Surg Oncol 1989;15:424-31.

Sahl WJ. Basal cell carcinoma: influence of tumour size on mortality and marbidity. Int J Dermatol 1995;34:319-21.

Sahl WJ. Basal cell carcinoma and lifestyle characteristics. Int J Dermatol 1995;34: 398-402.

Satinoff MI, Wells C. Multiple basal cell naeus syndrome in ancient Egypt. Med Hist 1969;13:294-7.

Scharfenberg $\mathbf{K}$, Wagner $R$, Wagner KG. The cytotoxic effect of ajoene, a natural product from garlic, investigated with different cell lines. Cancer Lett 1990;53:103-8.

Schnelter M, Krebs A. Therapeutische, funktionelle und kosmetische Spätergebnisse von 103 Patienten mit mittels Weichstrahltherapie behandelten 117 Basaliomen. Dermatologica 1982;165:342-51.

Schoenfeld N, Mamet $R$, Epstein $O$ et al. The heme biosynthesis pathway in the regenerating rat liver. The relation between enzymes of the heme synthesis and growth. Euro J Biochem 1987; 166:663-6.

Schoenfeld $\mathbb{N}$, Mamet $R$, Leibovici $L$ et al. Growth rate determines activity of $P B G$ deaminase in non-malignant and malignant cells. Biochem Metab Biol 1988;40:213-7.

Schrijvers CT, Stronks $K$, wan der Mheen $D H$ et al. Validation of cancer prevalence data from a postal survey by comparison with cancer registry records. Am $J$ Epidemiol 1994; 139:408-14.

Schuller DE, Berg JW, Sherman $G$ et al. Cutaneous basosquamous carcinoma of the head and neck; a comparative analysis. Otolaryngol Head Neck Surg 1997;87:420-7.

Schwartz $\mathbf{S K}$, Absolon $K$, Vermund $H$. Some relationships of porphyrins, $x$-rays and tumours. Univ Minn Med Bull 1955;27:7-8.

Sexton M, Jones $D B$, Maloney $M E$ et al. Histologic pattern analysis of basal cell carcinoma: study of a series of 1039 consecutive neoplasms. J Am Acad Dermatol 1990; 23:1118-26.

Shannon RL, Strayer DS. Arsenic-induced skin toxicity. Human Toxicol 1989;113:99104.

Silverman MK, Kopf $A W$, Grin $C M$ et al. Recurrence rates of treated basal cell carcinomas. Part 1: Overview. J Dermatol Surg Oncol 1991(a);17:713-8.

Silverman MK, Kopf AW, Grin CM et al. Recurrence rates of treated basal cell carcinomas. Part 2: Curettage-electrodesiccation. I Dermatol Surg Oncol 1991(b);17:720 6.

Silverman MK, Kopf AW, Bart RS et al. Recurrence rates of treated basal cell carcinomas. Part 3: Surgical excision. J Dermatol Surg Oncol 1992 (a); 18:471-6.

Sulverman MK, Kopf AW, Gladstein AH et al. Recurrence rates of treated basal cell carcinomas. Part 4: X-ray therapy. J Dermatol Surg Oncol 1992 (b); 18:549-54.

Soler AM, Warloe $T$, Tausjo $J$ et al. Photodynamic therapy by topical aminolevulinic

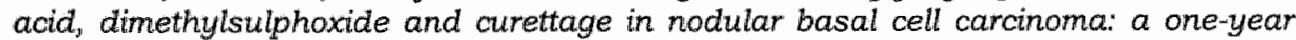
follow-up study. Acta Derm Venereol 1999;79:204-6.

Spiller WF. Combination curettage and cryosurgery for basal cell carcinoma. Syllabus for basic cryosurgery course. American Academy of Dermatology, Annual meeting 1990;26-7.

Stange PR, Lange PG. Long-term management of basal cell neus syndrome with topical tretinoine and 5-fluorouracil. I Am Acad Dermatol 1992;27:842-5.

Staples $\mathbf{M}_{3}$ Marks $R$, Giles $G$. Trends in the incidence of non-melanocyic skin cancer 
(NMSC) treated in Australia 1985-1995: are primary prevention programs starting to have an effect? Int $J$ Cancer 1998:78:144-8.

Stern RS. The mysteries of geographic variability in non-melanoma skin cancer incidence. Arch Dermatol 1999;135:843-4.

Stone $\mathbf{J L}$, Reizer $\mathrm{G}$, Scotto I. Incidence of nonmelanoma skin cancer in Kauai during 1983. Hawaii Med 1986;45:281-6.

Svaasand Lo, Tromberg $B U$, Wyss $P$. Light and drug distribution with topically administered photsensitizers. Lasers Med Sci 1996;11:261-5.

Svanberg $\mathbf{K}$, Andersson $T_{0}$ Killander $D$, et al. Photodynamic therapy of non-melanoma malignant tumaurs of the skin using topical d-aminolevulinic acid sensitization and laser irradiation. Br J Dermatol 1994;130:743-51.

Svanberg $\mathbf{K}$, Liu $D L_{*}$ Wang $I$ et al. Photodynamic therapy using intravenous daminoleunlinic acid-induced protoporphyrin $I X$ sensitisation in experimental hepatic tumours in rats. Br J Cancer 1996;74:1526-33.

Szeimies RM, Sassy $T$, Landthaler $M$. Penetration potency of topical apllied 5-aminolevulinic acid for photodynamic therapy of basal cell carcinoma. Photochem Photobiol $1994 ; 59 ; 73-6$.

Szeimies RM, Abels $C$, Fritsch $C$. Wavelength dependency of photodynamic effects after sensitization with 5-aminolevulinic acid in vitro and in vivo. I Invest Dermatol 1995; 105:672-7.

Szeimies RM, Karrer S, Sauerwald $A$, et al. Photodynamic therapy with topical application of 5-aminolevilinic acid in the treatment of actinic keratoses: an initial clinical study. Dermatology 1996;192:246-51.

Tappeiner von $\mathbf{H}$, Jesionek $A$. Therapeutische Versuche mit fluorescierenden Stoffen. Munch Med Wochenschr 1903;47:2042-4.

Tappeiner von $\mathbf{H}$, Jodlbauer $A$. Ueber Wirkung der photodynamischen fluorescierenden) Stoffe auf Protozoan und Enzyme. Dtsch Arch Klin Med 1904;80:427-87.

Tappeiner von $\mathbf{H}$, Jodlbauer A. Die sensibilisierende Wirkung fluorescierender Substanzer. Gesammte Untersuchungen uber die photodynamische Erscheinung. $F C W$ Vogel, 1907, Leipzig.

Thissen MR, Neumann HA. The sense and non-sense of immunohistochemical staining in basal cell carcinomas and other cutaneous tumours. Skin Cancer 1997;12:3742.

Thissen MR, Neumann $H A$, Berretty $P J$ et al. The treatment of basal cell carcinoma patients by dermatologists in the Netherlands. Ned Tijdschr Geneeskd 1998;142: 1.563-7.

Thissen MR, Neumann HA, Schouten LI. A systematic review of treatment modalities for primary basal cell carcinomas. Arch Dermatol 1999;135:1177-83.

Thissen MR, Schroeter CA, Neumann HA. Effective photodynamic therapy with 5aminolevulinic acid for nodular basal cell carcinomas using a preceding debulking technique. Br J Dermatol 2000;142:338-9.

Torre D. Cryosurgical treatment of epitheliomas using the cone-spray technique. $J$ Dermatol Surg Oncol 1977;3:432-6.

Torre D. Cryosurgical instrumentation and depth dose monitoring. In: Breithart $E_{\text {, }}$ Dachow-Siwiec $E$ (eds). Clinics in dermatology: Advances in cryosurgery. Elsevier, New York, 1990:59.

Tromovitch TA, Stegeman SJ. Microscopically controlled excision of skin tumours. Arch Dermatol 1974;110:231-2.

Tromovitch TA, Stegeman SJ. Microscopic-controlled excision of cutaneous tumours. Cancer 1978;41:653-8.

Tuppurainen $\mathbf{K}$. Cryotherapy for eyelid and periocular basal cell carcinomas: out- 
come in 166 cases over an 8-year period. Graefes Arch Clin Exp Ophthalmol 1995: 233:205-8.

Veen van der $\mathbf{N}$, Leengoed $v H L$, Star WM. In vivo fluorescence kinetics and photodynamic therapy using 5-aminolevulinic acid-induced porphyrin; increased damage after multiple irradiations. Br J Cancer 1994;70:867-72.

Veen van der N, de Bruijn HS, Star WM: Photobleaching during and re-appearance after photodynamic therapy of topical ALA induced fluorescence in UVB-treated mouse skin. Int $J$ Cancer 1997;72:110-8.

Veen van der $\mathbf{N}$. Mechanism and optimization of PDT using ALA induced PpLX. Thesis 1999, Erasmus University Rotterdam.

Veen van der $\mathrm{N}$, Hebeda $K M$, de Briijn $H S$, et al. Photodynamic effectiveness and vasoconstriction in hairless mouse skin after topical 5-aminoleutlinic acid and singleor two-fold illumination. Photochem Photobiol (in press).

Wade TR, Ackermann AB. The many faces of basal cell carcinoma. I Dermatol Surg Oncol 1978;4:23-8.

Warloe T, Peng Q, Steen HB et al. Localization of porphyrins in human basal cell carcinoma and normal sicin tissue induced by topical application of 5-aminalevulinic acid. In: Spinelli $P$, Dal Fante $M$, Marchesini $R$. Photodynamic therapy and biomedi-cal lasers, Elsevier Science Publishers BV, Amsterdam, 1992:454-8.

Wei Q. Matnoski GM, Farmer ER et al. DNA repair and susceptibility to basal cell carcinoma: a case control study. Am J Epidemial 1994;140:598-607.

Weinstock MA. Non-melanoma skin cancer mortality in the United States, 1969 through 1988. Arch Dermatol 1993;129;1286-90.

Wennberg A, Lindholm $L E$, Alpsten $M$, et al. Treatment of superficial basal cell carcinomas using topically applied delta-aminoleurlinic acid and filtered xenon lamp. Arch Dermatol Res 1996;288:561-4.

Wikonkal NM, Berg $R J$, van Haselen CW. Bcl-2 us p53 protein expression and apoptotic rate in human nonmelanoma skin cancers. Arch Dermatol 1997;133:599-602.

Wildsmith JA, Strichartz GR. Local anesthetic drugs - a historical perspective. $\mathrm{Br} J$ Anaesth 1984;56:937-9.

Wilson BD, Mang TS, Stoll $H$ et al. Photodynamic therapy for the treatment of basal cell carcinoma. Arch Dermatol 1992;128:1597-1601.

Wolf DJ, Zitelli JA. Surgical margins for basal cell carcinoma. Arch Dermatol 1987; 123:340-4.

Wolf $\mathbf{P}$, Rieger E, Kerl H. Topical photodynamic therapy with endogenous porphyrins after application of 5uminolevulinic acid; an alternative treatment modality for solar keratosis, superficial squamous cell carcinomas and basal cell carcinomas ? $J \mathrm{Am}$ Acad Dermatol 1993;28:17-21.

Yalkubu A, Mabogunje OA. Skin cancer in Zaria, Nigeria. Trop Doct 1995;25:63-7.

Yang D, Morrison BD, Vandongen $Y K$ et al. Malignancy in chronic leg ulcers. Med $J$ Aust 1996;164:718-20.

Zacarian SA. Cryosurgery of cutaneous carcinomas: An eightteen year study of 3022 patients with 4028 carcinomas. I Am Acad Dermatol 1983;9:947-56.

Zacarian SA. Cryogenics: the cryolesion and the pathogenesis of cryonecrasis. In: Zacarian SA, ed. Cryosurgery for skin cancer and cutaneous disorders. St Louis: CV Mosby, 1985:1-30.

Zacarian SA. Cryasurgery for skin cancer. In: Zacarian SA, ed. Cryosurgery for skin cancer and cutaneous disorders. St Louis: CV Mosby, 1985:96-162.

Zanetti R, Rosso S, Martinez $C$ et al. The multicentre south European study "Helios" I: Skin characteristics and sunburns in basal cell and squamous cell carcinomas of the skin. Br J Cancer 1996;73:1440-6. 


\section{LIST OF ABBREVIATIONS}

$\begin{array}{ll}(5 / \text { d- }) A L A & :(5 / \text { delta-jaminolevulinic acid } \\ B & : \text { beautician } \\ B C C & : \text { basal cell carcinoma } \\ C E & : \text { curettage/ electrodesiccation } \\ C S & : \text { cryosurgery } \\ C Y P & : \text { cytochrome } P 450 \\ D(f) & : \text { female dermatologist } \\ D(m) & : \text { male dermatologist } \\ D N & : \text { dermatologic nurse }\end{array}$

FWHM: full width at half maximum

\begin{tabular}{|c|c|}
\hline$G S T$ & : glutathione S-transferase \\
\hline IM & : immunotherapy \\
\hline$i . m$ & : intramuscular \\
\hline i.t. & : intravenous \\
\hline$J$ & : Joule \\
\hline MMS & : Mohs' micrographic surgery \\
\hline$m W$ & : milliWatt \\
\hline $\mathrm{nm}$ & : nanometer \\
\hline NMSC & : non-melanoma skin cancer \\
\hline$P$ & : patient \\
\hline$P B G D$ & : porphobilinogen deaminase \\
\hline$P D T$ & : photodynamic therapy \\
\hline $\operatorname{PpLX}$ & : protoporphyrin $L X$ \\
\hline$P S$ & : plastic surgeon \\
\hline$P U V A$ & : psoralen-ultraviolet $A$ \\
\hline$R T$ & : radiotherapy \\
\hline$S C C$ & : squamous cell carcinoma \\
\hline$S E$ & : surgical excision \\
\hline s.e.m. & : standard error of mean \\
\hline$U V(A / B)$ & : ultra violet $(A / B)$ \\
\hline us & : versus \\
\hline$w / o$ & : water in oil \\
\hline
\end{tabular}




\section{CURRICULUM VITAE}

Monique Thissen werd op 1 oktober 1966 te Sittard geboren. In deze plaats heeft zij de middelbare schoolopleiding gevalgd op de toenmalige r.k. Schalengemeenschap "Serviam". Na het behalen van het Gymnasium-B diploma, begon zij in september 1985 aan haar studie Geneeskunde aan de Rijksuniversiteit Limburg te Maastricht, alwaar zij in 1989 "cum laude" slaagde voor het doctoraal examen en twee jaar later, in september 1991, de artsenbul in ontvangst mocht nemen.

De eerste zelfstandige erwaring in de gezondheidszorg deed zij op als agniopsychiatrie op de PAAZ-afdeling van een perifeer ziekenhuis. De voorliefde voor de "Aandoeningen van de Huid (de spiegel van de ziel)" had zij echter reeds gedurende de basisopleiding ontwikkeld, en het was in ditzelfde ziekenhuis warar zij contact legde met de maatschap van dermatologen (Dr.M.Go, Dr.u. White, Drs.J.Lambers, Drs.K. Vissers-Croughs), om vervolgens van 1992 tot 1994 als arts-assistent binnen deze vakgroep de grondbeginselen van de dermatologie te leren.

Omdat de opleidingsplaatsen voor dermatologie schaars waren, vertrok aij in het najaar van 1994 naar Amsterdam waar zij collega Dr. W. Westerhof bijstond in de startfase van het super-specialistisch Nederlands Instituut voor Pigmentstoornissen. Op deze werkplek heeft zij zowel patiëntenzorg als wetenschappelijk onderzoek verricht, en met de hieruit voortvloeiende publicaties op zak heeft zij vervolgens in september 1995 gesolliciteerd naar een opleidingsplaats voor dermatologie in het Academisch Ziekenhuis Maastricht (opleider: Prof.dr.H.A.M.Neumann).

Er resteerde echter nog een wachttijd uan twee jaar, die zij welbesteed doorbracht als agnio-interne geneeskunde in het St.Maartensgasthuis te Venlo en aansluitend bij de afdeling dermatologie van het Catharina Ziekenhuis te Eindhoven bij Dr.P.Berretty en Drs.A.Ideler. Het was op deze laatste plek dat reeds een aanvang gemaakt werd met meerdere studies van het promotie-onderzoek betreffende de behandeling wan het carcinama basocellulare.

In november 1997 kon zij officiêel beginnen met de specialisatie tot dermatovenereoloog in het Academisch Ziekenhuis Maastricht. Inmiddels is zij per 1 januari 2000 ook gestant met de aanvullende opleiding voor Mohs' chirurg. 


\section{LIST OF PUBLICATIONS}

Thissen MR, Westerhof $W$. Postinflammatoire kleurveranderingen van de huid. Ned Tijdschr Dermatol Venereol 1995;5:55-6.

Thissen MR, Westerhof $W$. Laserbehandeling van gepigmenteerde huidafwijkingen. Ned Tijdschr Dermatol Venereol 1996:6:126-9.

Thissen MR, Westerhof $W$. Laser treatment for further depigmentation in vitiligo. Int $J$ Dermatol 1997;36:386-8.

Thissen MR, Westerhof W. Lentigo maligna treated with Ruby laser. Acta Dermato Venereol 1997;77:163.

Thissen MR, Neumann HA. The sense and non-sense of immunohistochemical staining in basal cell carcinomas and other cutaneous tumours. Skin Cancer 1997;12: 3742.

Thissen MR, Neumann HA, Berretty PJ et al. The treatment of basal cell carcinomas by dermatologists in the Netherland's. Ned Tijdschr Geneeskd 1998;142:1563-7.

Thissen MR, Koedam MI. Geagmineerde en gedissimineerde cutane piloleiomyomen. Ned Tijdschr Geneeskd 1998;142:2707-8.

Thissen MR, Schroeter CA, Neumann HA. Photodynamische therapie als behandeling van het basaalcelcarcinoom. Ned Tydschr Dermatol Venereol 1998;8:171-2.

Thissen MR, Koedam IM. Geagmineerde en gedissimineerde cutane piloleiomyomen. Ned Tijschr Dermatol Venereol 1998;8:134-5.

Thissen MR, Jonkman M, Moogh U. Epidermolysis bullosa met vlekkige pigmentatie. Ned Tijdschr Dermatol Venereol 1998;8:152-3.

Thissen MR, $v$ Beek $J$, v Mierlo P. Acrokeratosis paraneoplastica van Bazex. Ned Tyjdschr Dermatol Venereol 1998;8:168-9.

Thissen MR, Neumann HA, Berretty PJ et al. Behandeling van het basalecelcarcinoom door dermatologen in Nederland. WCS News 1999;15:35-8.

Thissen MR, Newmann HA, Schouten LW. A systematic review of treatment modalities for primary basal cell carcinomas. Arch Dermatol 1999;135:1177-83.

Thissen MR, Schroeter CA, Neumann HA. Effective photodynamic therapy with 5aminolevulinic acid for nodular basal cell carcinomas using a preceding debulking technique. Br J Dermatol 2000; 142:338-9. 


\section{DANKWOORD}

"Promoveren doe je niet alleen". Toen ik tegen thet einde van rit de namen van diegenen die mij bij deze inspanning hebben bijgestaan op papier probeerde te krigen, was ik toch wel verrast over de omvang van deze lijst. Allemaal personen die mij op hun eigen unieke wijze gesteund hebben, sommigen wat meer en anderen wat minder, maar ze waren er toch voor (en met) mij, want promoveren kun je niet alleen. Een gemeenschappelijke kenmerk van promoveren en van Mohs' chirurgie is dat je aanvankelijk wel weet waar je begint, maar dat je niet weet waar je zult eindigen, en. op elk moment kan het zo zijn dat je hulp nodig hebt. Door de omvang van de lijst is het onmogelijk om iedereen op deze plaats persoonlijk te bedanken, maar ik will toch tot enkele personen een woord van dank richten.

Dit dankwoord is misschien anders dan verwacht, maar ik ben van mening dat de eerste woorden toebehoren aan mijn "thuisfront" dat toch als basis voor mijn functioneren garant staat. Pappa en mamma, bedankt dat jullie mij vanaf het allereerste moment tot de dag van vandaag in alle opzichten hebben willen en kunnen steunen, zowel in de goede als de (sporadisch voorkomende) minder goede tijden; slechte tijden heb ik gelukkig niet gekend, want "ik ben voor het geluk geboren" zoals jullie altijd zeiden, en daar ben ik het roerend mee eens. Jack, jij kwam logischerwijze pas op een later moment mijn leven binnenwandelen, maar ik ben er vast van overtuigd dat het hele promotie-traject veel langer en moeizamer geweest zou zijn zonder jou. Behalve dat je steeds geinteresseerd bleef in mij en mijn onderzoek en ik steeds buj je terecht kon, ondanks je eigen drukke leven (werken, opleiding volgen en slagenl), was je een kei in het re-arganiseren van de chaos die ik steeds in huis organiseerde. Ik hoop dat we samen nag eens aan een gestructureerd bestaan toekomen. Bennett en Beau, ik vind het onvoorstelbaar knap dat jullie het "honde-leven" bij ons op een. enkele blafpartij na zo onvoorwaardelijk geaccepteerd hebben, en vervolgens ook nog eens als vaetenwarmer fungeerden bij het schrijwen wan dit boekje, hetgeen toch een behoorlijke kluif was (waf 1... kluif ? waar 2); ik zal er vaker en meer voor jullie proberen te zijn in de toekomst (en dat geldt ook voor jou, Boyke).

Een bijzonder woord van dank wil ik veruolgens richten tot mijn promotor en opleider Prof.dr.H.A.M.Neumann. Beste professor, bedankt voor het feit dat U mij de mogelijkheid hebt gegeven om de fotodynamische therapie binnen onze vakgroep te introduceren en verder uit te bouwen. Op regelmatige tijden evalueerden wij de vorderingen van mijn proefschrift, waarbij het mij steeds weer verbaasde dat $U$ voor elk probleem een oplossing wist of een "uriendje" te hulp kon roepen; maar ja, $U$ bent dan ook niet voor niets professor. Toch liet $U$ mij telkens de mogelijkheid om het onderzoek op een persoonlijke manier gestalte te geven, hetgeen ik altijd erg gewaar 
deerd heb. Ook op anregelmatige tijden kon ik steeds bij $U$ aankloppen, en samen met uw echtgenote Julia liet $U$ Jack en mij zien hoe het noodzakelijke met het aangename te combineren is. Professor, bedankt voor Uw begeleiding en het in mij gestelde vertroutwen.

Professor Manni, professor Boeckx, professor Borgers, professor v.Gemert en doctor Star wil ik bedanken voor het feit dat zij bereid waren om deel uit te maken van de beoordelingscommissie. Willem Star wil ik nog extra bedanken voor de interessante discussies over photadynamische therapie en voor het kritisch lezen van twee manuscripten.

Mieke, zonder jou en je achterban van de apotheek in het Catharina Ziekenhuis, zou de intracutane injectietechnique voor photodynamische therapie niet zo zorguuldig van de grond gekomen zijn. Bedankt voor je aanvankelijke weerstand tegen mijn verzokk om "even wat ALA op te lossen", en natuurlijk voor de enorme steun en prettige samenwerking (en gezellige avonden) nadien.

Dr. Berretty, Drs. Ideler en Adriënne, bedankt voor jullie moeite om samen met mij al die patiënten voor de studies te "verzamelen" en te "overtuigen" dat die "nieuwe behandeling" echt helemaal het einde zou zijn.

Careen Schroeter, bedankt voor alle moeite en het op-en neer reizen tussen Eindhoven en Maastricht met de Versaitight; het blijft toch de "Rolls Royce" onder de lichtbronnen.

Dominic en Riëtte, ontzettend bedankt voor de bereidheid om jullie meet-apparatuur naar Maastricht te verplaatsen, en voor jullie inzet en uithoudingsvermogen om dag-in, dag-uit, van 's morgens uroeg tot 's avonds laat met mij fluarescenties te meten; het was echt beestachtig en vermoeiend.

Coen, Joyce, May, Frans, Hub, Kurt-Jan en Judith (en alle "babes"), bedankt voor jullie hulp buj de dierstudies; het was geen gemakkelijke opgave.

Richard Dutrieux, het leek wel of je de enige patholoog in Nederland was die de coupes van de dierstudie wilde snijden en beoordelen. Bedankt voor je inzet.

Leo Schouten, Patty Nelemans en Fred Nieman, dit boekje is mede tot stand gekomen door jullie epidemiologische en statistische ondersteuning. Mijn welgemeende dank hiervoor.

Vervolgens wil ik mijn collega's van de afdeling dermatalogie wan het AZM, mijn ex-collega's (artsen, verpleegkundigen en secretaresses) van de afdeling dermatologie van het Catharina Ziekenhuis te Eindhoven en alle patienten die aan de verschillende onderzoeken hebben deelgenomen bedanken voor hun bereiduilligheid, hulp en inzet (Bas, voor jou een extra woord van dank voor het overnemen van 
een aantal spreekuren op maandagochtend).

Mijn paranimfen, Mieke de Blois en Gertruud Krekels, wil ik bedanken voor hun steun bij deze promotie.

Aan alles komt een einde, zo ook aan dit boekwerk. Maar dat betekent niet het einde van mijn wetenschappelijke carrière. Met name op het gebied van de fotodynamische therapie zijn er nog talloze facetten die verder uitgediept kunnen en moeten worden om deze therapie te optimalizeren. Danielle, ik ben dan ook heel blij dat jij bereid bent om deze onderzoekslijn voort te zetten en ik hoop dat we samen nog een heel eind komen. 